\title{
Redressing the Ocularcentric Culture of architectural design: A Phenomenological and Participatory Approach in VR
}

By Elisaveta Boulatova, B.Arch. Sc., Ryerson University 2015

A thesis project presented to Ryerson University

In partial fulfillment of the requirements for the degree of

Master of Architecture in the Program of Architecture

Toronto, Ontario, Canada, 2018

(c) Elisaveta Boulatova, 2018 


\section{AUTHOR'S DECLARATION}

I hereby declare that I am the sole author of this thesis. This is a true copy of the thesis,including any required final revisions, as accepted by my examiners.

I authorize Ryerson University to lend this thesis to other institutions or individuals for the purpose of scholarly research.

I further authorize Ryerson University to reproduce this thesis by photocopying or by other means, in total or in part, at the request of other institutions or individuals for the purpose of scholarly research.

I understand that my thesis may be made electronically available to the public. 


\title{
Redressing the Ocularcentric Culture of architectural design: A Phenomenological and Participatory Approach in VR
}

\author{
Elisaveta Boulatova
}

Master of Architecture 2018

Architecture Program, Ryerson University

\begin{abstract}
In the development of architectural representation, the image has become a metonym of architectural experience. This productive tension is maintained by the expectation of fragmented representation to identify and reproduce the experiential and phenomenological dimensions perceived in the physical experience with architecture. Returning to the investigation of representation and perception exemplifies that each new technology introduces a new scale of extension in both design capability and opportunity for feedback. The degree of immersivity introduced by virtual reality technologies elicits several scales of experience. This thesis uses virtual prototyping in the design stage to elicit a product that is not a reflection of intention, but a result of something discovered, evaluated and experienced in immersion prior to realization. An increase in receptivity to the qualities of the design and its context relative to the perceiver has the potential to change the relationship of digital space to digital place.
\end{abstract}




\section{ACKNOWLEDGMENTS}

I would like to express my sincere gratitude to many. Vincent Hui, my supervisor, for his continuous belief in me, and for helping me focus on the scope of this work. Kendra Schank Smith, my second reader, for raising important questions to guide the research between the blurry lines of art and architecture. John Cirka, for his commentary, guidance and invaluable feedback. Scott Sorli, Paul Floerke and Marco Polo, for teaching and sharing their knowledge of ideas, places and direction, and who each in their own way, are the best possible guides on architecture tours. Jason Ramelson, Jordan So, Frank Bowen, Luisa Gonzalez, Jigar Solanki and Aris Peci-their help in this process and the in the project that stalled this one is immeasurable. I owe many thanks to everyone who volunteered, contributed or gave a helping hand. My list of gratitude continues to grow larger everyday. 
To Karin, Sasha, Tanya and Marik, who always teach me by example, love and humour 
TABLE OF CONTENTS

Author's Declaration

Abstract

Acknowledgements

Dedication

List of Figures

List of Appendices

Introduction: Digital Space and digital place

PART ONE: REPRESENTATION

01 Verbal to Visual to Spatial (but tactile) perspective

02 Vitual realities: Immersion, Application and Prototyping

\section{PART TWO: PERCEPTION}

03 Sensory and Information based Theories of perception

\section{Part Three: Design Project}

06 Approach: The Four Scales

07 Narrative Scale: The experience

08 Immersive Scale: Environment to be perceived

09 Building Scale: Information for perception

10 Relational Scale: Activity of perception 


\section{LIST OF FIGURES}

Figure 1.1. Raphael, School of Athens fresco. Photograph by xvi Author

Figure 1.2 Raphael's fresco. Photograph by Author

Figure 1.3 Retrived from: https://www.khanacademy.org/ humanities/art-1010/wwi-dada/art-great-war/a/italianfuturism-an-introduction

Figure 1.4 Retrived from: https://www.pablopicasso.org/ avignon.jsp

Figure 1.5 Retrived from: http://arthistoryguide.blogspot. ca/2008/01/cubism.html

Figure 1.6 Retrived from: http://www.mascontext.com/ issues/30-31-bilbao/turning-point-two-guggenheimmuseum-bilbao/

Figure 1.7 Presence versus telepresence. Image by Author. 4

Figure 2.1 Mirror experiment. Retrived from: http://www. chasrowe.com/history/images/baptistery2.jpg

Figure 2.2 Smithsonian 3D scanned artifact. Retrived from: https://3d.si.edu/

Figure 2.3 Drone earthquake assesment. Retrived from:

Figure 3.1 Osmose, 1995. Retrived from: http://www. immersence.com/osmose/os_treepond.html

Figure 3.2 Degrees of Freedom. Retrived from: http://www. 20 leadingones.com/articles/intro-to-vr-4.html

Figure 3.3 Lytro stills from video. Retrived from: http://www. 21 lytro.com
Figure 3.4 First computer-driven, head-mounted viewing 22 device Image retrived from: Ellis, Stephen. Pictorial Communication In Real And Virtual Environments. University of Utah, Salt Lake City, Utah, 2003

Figure 3.5 Inductrial Application. Image retrived from: Dai, 23 Fan. Virtual Reality for Industrial Applications. Springer, 1998. p18

Figure 3.6 Prototyping in construction design. Retrived from: Brandon, S. Peter, Kocaturk, Tuba. "Design, Engineering and manufacturing challenges" Virtual Futures for Design, Construction and Procurement, 2018. p. 36)

Figure 3.7 Physical Model. Photograph by Author.

Figure 3.8-3.9 Yulio cubemap Retrived from: https://yulio. 26 zendesk.com/hc/en-us/articles/219131167

Figure 3.10 Virtual applications before 2000. Retrived from: Mine R.M., Brooks, F., Sequin, H.C., Moving Objects in Space: Exploiting Proprioception in In Virtual Environment Interaction, University of North Carolina at Chapel Hill, University of Berkeley, 2001

Figure 4.1 Spotlight model of attention. Retrived from: https://upload.wikimedia.org/wikipedia/commons/ thumb/4/46/Wikipedia-spotlight.jpg/200px-Wikipediaspotlight.jpg

Figure 4.2 Field of consciousness model. Retrived from: http://www.jstor.org/stable/40320393

Figure 4.3 Kimbell Art Museum. Retrived from: https://www. 36 archdaily.com/123761/ad-classics-kimbell-art-museumlouis-kahn 
Figure 4.4 Sainte Marie de la Tourette, Le Corbusier Retrived 36 from: http://numelyo.bm-lyon.fr/f_eserv/

Figure 4.5 Yulio Heatmap. Retrived from: Yulio.com (https:// yulio.zendesk.com/hc/en-us/articles/219131167)

Figure 4.6 Snarkitexture. Retrived from: https://www. dezeen.com/2017/08/31/snarkitecture-formantasmawashington-street-installations-exhibit-columbus/

Figure 4.7 Ames room plan. Retrived from: https://upload. wikimedia.org/wikipedia/commons/thumb/8/88/Ames_ room.svg/1200px-Ames_room.svg.png

Figure 4.8 Parti plan. Image by Author.

Figure 4.9 The Aventine Keyhole. Photograph by Author.

Figure 5.1 Domus Dei Holy Heart Church facade detail. Image retrived from: Bleeckere, Sylvain de, and Sebastiaan Gerards. Narrative Architecture: a Designers Story. Routledge, 2017.

Figure 5.2 Andrei Tarkovsky on set of Nostalgia. Retrived from: https://i.pinimg.com/564x/0c/64/93/0c6493e63ad1b0 b8bba37476ec91213f.jpg

Figure 5.3 Mark Foster Gage Architects concept render. Retrived from: https://s-media-cache-ak0.pinimg.com/origin als/82/9e/6e/829e6ec4a4ae8724dd6adf9a68b8e22f.jpg

Figure 5.4 Skin. Retrived from: https://i.pinimg.com/564x/6f /89/0e/6f890e758e49f3a800f3d08a22dded63.jpg

Figure 6.1 Le Corbusier Marseille block is 'four man' tall. Image retrived from: Rasmussen, Steen Eiler. Experiencing Architecture. MIT Press, 1959.

Figure 6.2 Distance-size perception. Image retrived from: Gordon, Ian E. Theories of Visual Perception. Taylor and Francis, 2004.
Figure 7.1 Existing context. Image by Author.

Figure 7.2 Three chapel components diagram. Image by Author

Figure 7.3 Design Axo. Image by Author.

Figure 8.1 Via Giulia site from bird's eye view. Provided by: http://student.archmedium.com/

Figure 8.2-8.3 Pantheon. Photograph by Author.

Figure 8.4 Piazza Navona. Photograph by Author.

Figure 8.5 Map of Rome. Image by Author. Base retrived from: ARCMedium, CAD file.

Figure 8.6 The Possesso route. Retrived from: Mayernik 68.

Figure 8.7-8.8 San Giovanni dei Florentini church. Images by Author.

Figure 8.9 San Fillippo Neri facade before demolition. Retrived from: https://www.romasparita.eu/foto-romasparita/files/2011/05/romasparita_4809.jpg

Figure 8.10 San Fillippo Neri facade, 1993. Retrived from: http://romafelix.com/sfilineri.htm

Figure 8.11-8.18 Site photos. Photography by Author.

Figure 8.19 Via Giulia context fragment. Image by Author.

Figure 8.20-8.22 Site photos. Photography by Author.

Figure 8.23 Nolli map of site. Image by Author. Base retrived 82 from: https://upload.wikimedia.org/wikipedia/commons/ thumb/c/c7/Nolli_1748_San_Filippo_Neri_in_Via_Giulia. JPG/397px-Nolli_1748_San_Filippo_Neri_in_Via_Giulia.JPG Figure $\mathbf{8 . 2 4}$ Nolli map of new design. Image by Author. 
Figure $\mathbf{8 . 2 5}$ Site piazza diagram. Image by Author.

Figure 8.26 Threshold diagram. Image by Author.

Figure $\mathbf{8 . 2 7}$ Site plan. Image by Author.

Figure 8.28 Piazza diagram. Image by Author.

Figure 8.29 Videocapture 1. Video by Author.

Figure 9.1-9.2 Cafe Entrance. Images by Author.

Figure 9.3-9.8 Cafe Entrance FPRs. Images by Author.

Figure 9.9-9.10 Cafe Render. Images by Author.

Figure 9.11 Lower level plan. Images by Author.

Figure 9.12 Floorplans. Images by Author.

Figure 9.13 Video Capture 2, cafe interior. Video by Author.

Figure 10.1 Diagram plan. Image by Author.

Figure 10.2 Tempietto and Pantheon oculus comparison. Image by Author. Base retrived from: https://i.pinimg. com/564x/06/21/16/062116b84afbdac38cd79f1b3 1c366fd.jpg, https://i.pinimg.com/564x/d5/dc/04/ d5dc04ad15d844aaf62c2276c1a504f3.jpg

Figure 10.3 Pantheon and subbterenean gallery diameter comparison. Image by Author.

Figure 10.4-10.12 Renders of gallery space. Image by Author.

Figure 10.13 FPR of gallery space. Image by Author.

Figure 10.14 Basement plan. Image by Author.

Figure 10.15-10.20 Renders of gallery space. Images by

Author.

Figure $\mathbf{1 0 . 2 1}$ Videocapture of gallery space. Video by Author. 104

Figure 11.1-11.2 Casanova+Hernandez Lausanne Jardins. 108 Retrived from: http://www.casanova-hernandez.com/

Figure 11.3-11.4 Aventine Keyhole. Photography by Author.

Figure 11.5 Video Capture 5. Video by Author.

Figure 11.6 Light studies screencaptured FPRs. Images by Author.

Figure 12.1 Chapel interior from FPRs. Images by Author.

(1)




\section{LIST OF APPENDICES APPENDIX A}

Figure A.1-6 Site Photos. Photography by Author.

Figure A.7-10 Retrived from: Google Maps street view

Figure A.11-12 Iteration 1 site plan. Images by Author.

Figure A.13-14 Iteration 1 massing rendering. Images by Author.

Figure A.15 Aerial rendering. Image by Author.

Figure A.16 FPR Rendering. Image by Author.

Figure A.17 Rendering. Image by Author.

Figure A.18-21 FPR renderings. Image by Author.

Figure A.22 Section A-A. Image by Author.

Figure A.23 Physical Sectional Model. Photography by Author.

Figure A.24 Physical Model detail. Photography by Author.

Figure A.25 Model Section. Photography by Author.

Figure A.26 Physical Model. Photography by Author. 


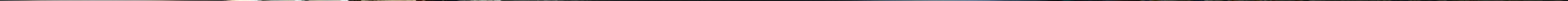




\section{Introduction}

Figure 1.1. Raphael, School of

Athens fresco at the Vatican in

Rome, Italy. Painted between

1509-1511 Figure 1.2 Raphael's

fresco on the opposite facing wall.

\begin{abstract}
"Architecture sets in stone, at least for a time, certain conceptions about the way the world is to be organized and human life is to be lived within in" - Murray A. Rae, Architecture and Theology
\end{abstract}

After Brunelleschi's re-discovered linear perspective circa 1420, virtually every painting in the Western tradition after the 15th century responded by either adopting perspectival rules or very consciously rejecting them-from futurism and cubism to expressionism and architectural deconstructivism. Nevertheless, perspective remains a dominant paradigm of our visual culture due to the domination of optical, mechanical and digital technologies producing endless multiplication of both still and moving images. In fact, the image has never been so cheap, and as Umberto Eco claims, in a state of malnourishment. ${ }^{1}$ However, vision is the only sense that is capable of keeping up with the increasing generation of ocular information. 

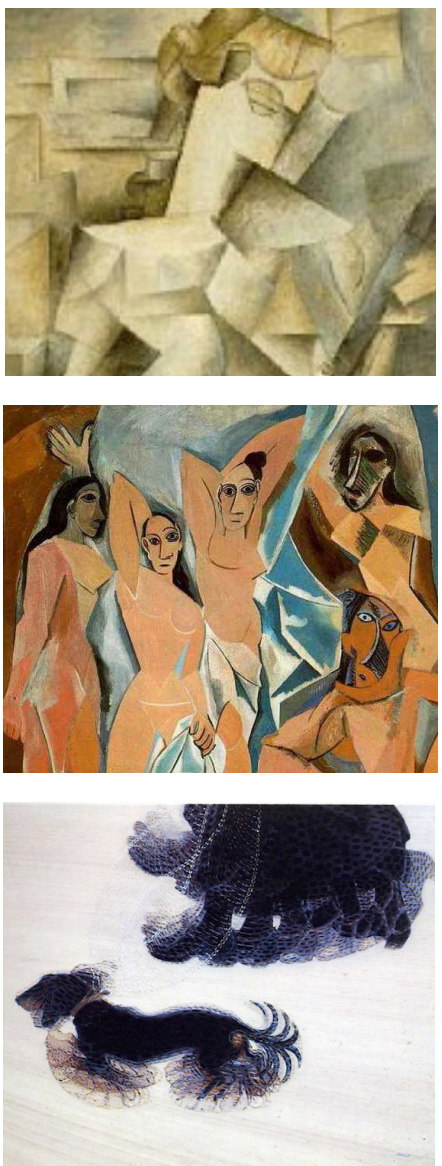

The liberation of the eye from the epistemology of Cartesian perspective can be demonstrated through the architectural and artistic styles in history':

The Baroque, eliminated the picture frame and vantage point by introducing asymmetrical diagonal compositions to create lateral tension between shapes.

The Renaissance, 'created space' by linking linear perspective to the size and placement of objects. However, Baroque artists created a greater illusion of depth by integrating foreshortening, diagonals and atmospheric perspective. Impressioninsts abandoned the boundary line, and instead balanced framing and perspectival depth. Cubists abandoned the single focal point to reactivate peripheral vision while reinforcing haptic experience Colour field painters rejected illusory depth 'in order to reinforce the presence of the painting itself as an iconic artifact and an autonomous reality ${ }^{\prime 3}$

The rise of digital technologies and new current models of visualization are slowly replacing photographic and perspectival imagery that were once revolutionary tools of invention. "In one generation, digital technologies have moved from word processing to image processing to 3-D processing-from verbal to visual to spatial operations"4 due to steady technical advancement creating ubiquity, efficiency and affordability of processing power. A 3-D scan can be taken as easily as a photograph, but contains a much greater amount of information. In practice, technical and design professionals have adopted 3D modeling over parallel projection, as there is no longer concern for information compression or storage. 3-D scanning and 3-D printing technologies are leading practices and making processes more efficient-effectively

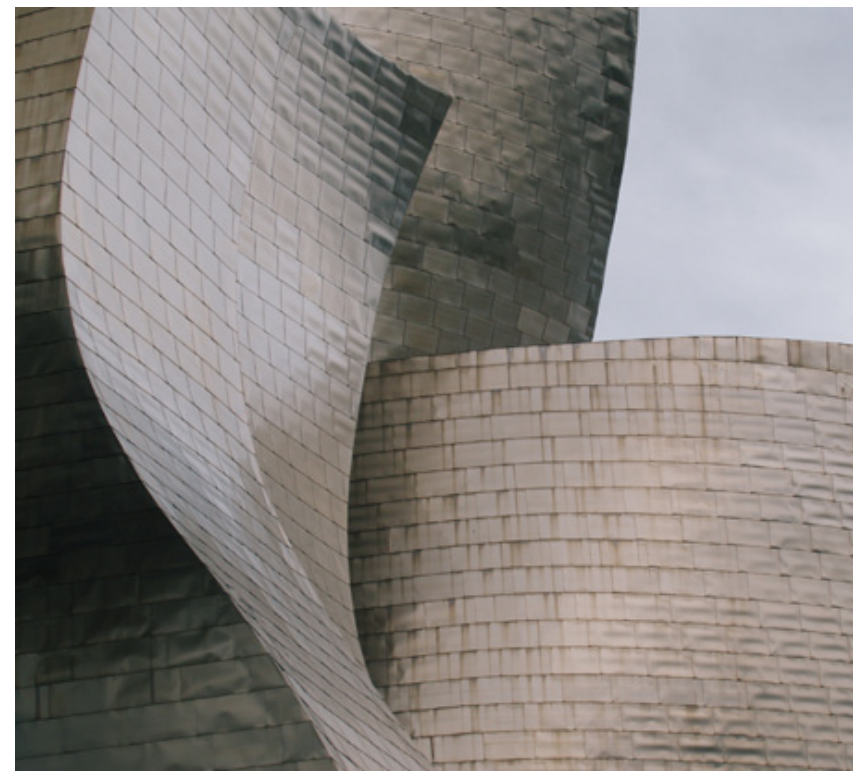

[LEFT TOP TO BOTTOM] Figure 1.3 Cubist painting by Georges Braque, Figure 1.4 Pablo Picasso, Five Women, Figure 1.5 A representation of an object's sensations. Giacomo Balla, Dynamism of a Dog on a Leash, 1912, oil on canvas, 35 1/2 x 43 1/4" (Albright-Knox Art Gallery, Buffalo), Figure 1.6 [ABOVE] Guggenheim Museum, Bilbao

reflected in the style of things we make. Digital designers and early adopters are positioned to 'capture, interpret, and give visible form to the technologies they use..5 New technologies, and with them the spirit of new ideas and possibilities, suggest the revolution of building.

"All tools modify the gesture of their users, and in the design professionals this feedback often leaves a visible trace... coalesce into the style of an age and express the spirit of a time."

The impact of this technological 'revolution' is imprinted in the radical forms of Frank Gehry's Guggenheim Bilbao. In 1997, the Bilbao was recognized as the symbol of a new technical age.

In a 2013 interview conducted by the Harvard Graduate 
School of Design, Denise Scott Brown reflected on the computerrelated role of photography in the processes of architecturefrom documentation to illustration to augmentation of design ideas and representation. She states that over the course of her career, "photography has evolved from a tool to a discipline of architecture,"7 creating a great amount of emphasis on twodimensional design in a three-dimensional practice. Due to visual dominance architecture has become, "an art of the printed image fixed by a hurried eye of the camera". However, representation for the sake representation, removes architecture from its core purpose, which is building:

'By furnishing this already crowded world with a duplicate one of images, photography makes us feel that the world is more available than it really is."

Representation, however, is a combination of content and context. It is a multivalent term that can mean 'to make present', suggesting the power to reveal something or render something visible. Or it can mean 'to stand in for something that is not there'10, acting on its behalf as a sign or witness. Ultimately, representation connects us to the world and encourages participation.

Nonetheless, digital technology continues to be a catalyst in the redefinition of practice, from computer-aided design (CAD) to Building Information Modeling (BIM). In the quest to help extend "the number of important operations which we can perform without thinking about them, we are amidst a battle for absolute control in our technological world."11 Digital modeling has combined numerous software tools into an individual design strategy, creating interaction with other disciplines, practices and techniques to show how space can be conceived. The contemporary process of architectural design is composed of a conventional set of projections-construction documents (used for accurate transcription), with projections at various scales from site context to details, altogether composing various parts of a dissected whole. ${ }^{12}$ The current state of technologically integrated design creates a framework where the aim of the project is to realize the final building as close as possible to the computed model, narrowing the gap of uncertainty in all areas of design and construction. In fact, the 'battle for control' suggests that we are "reaching a moment when the construction of technology should be allocated far more relevance than the technology of construction." 13

\section{The 'relatedness' to the world}

The process of design in screen-based space frames buildings as objects divorced from our sensory and information-based perception, as well as temporally or spatially distant from the physical environment. The digital landscape mediates the perception of environments where the three reference axes can be chosen at will, often at risk of presenting architecture as a 'finished product'. However, architecture exists in duration-contextual by nature, materially immersive, and immediate. ${ }^{14}$

For Finnish architect Juhani Pallasmaa, the essence of architecture is mediation, where buildings mediate between the world (the landscape) and us: 'composed of multiple essences that are both impure and messy. ${ }^{15}$ He claims that it is the tension between the utilitarian and poetic existence that gives architecture its power-in a combination of craft and art. In examining the nature of representation, beyond the conventional understanding of representational arts, (and beyond the image), there is an existing gap between architectural ideas and their physical representation of everyday experience.

The image is the basis 'experiential unit'. We relate images in order to understand represented space. William Whyte addresses the complexity of 'reading' buildings as 'archi-textures', citing Eco's argument that while, “we do commonly experience 


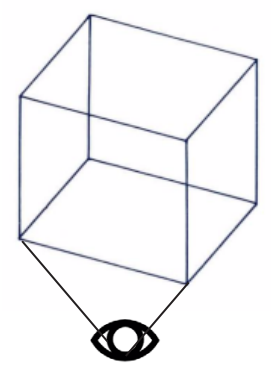

PHYSICAL SPACE

Physical (Model) Prototypes (PP)

Uses real physical objects to represent and interact with computer-generated information. Requires mental

translation of scale.

Tangible User Interface (TUI)

Using physical objects to create a

virtual model.

\section{PRESENCE:}

Immediate physical surroundings

\section{Bridging the Gulliver Gap}

The Gulliver Gap is a term coined by J. $M$ Anderson that refers to a 'toytown' syndrome, based around the limitations of a modelscope. It is "the awareness of our physical size in relation to that of a scale model" which highlights the ideal and the irony of our reality and a scaled model. ${ }^{16}$

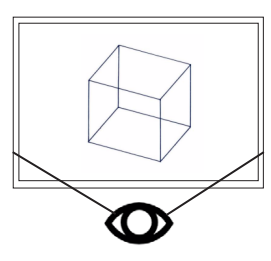

SCREEN-BASED SPACE

Desktop computer systems are

mediated by the screen. We cannot relate scale to our anatomy like we can to a physical models

\section{D modeling (Rhino/Revit)}

At the interface of the screen, the digital model is divorced from a fixed scale.

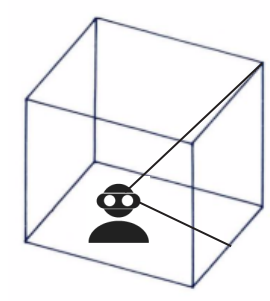

VIRTUAL SPACE

Virtual Reality (immersive) uses a head mounted display or projection based system, fills user's entire field-of-view. [Example: 'CAVE' (Cave Automatic Virtual Environment), HMDs, Google Cardboard]

In VR there is a relationship between the navigator, the scale of the digital model, and the viewer's perception.

\section{TELEPRESENCE:}

Virtual world
Figure 1.7 Presence versus telepresence in the types of work spaces 
architecture as communication, even while recognizing its functionality...The message however changes when we experience architecture as a plan, as a picture, in text, or as structure. In everyday life, and as historians, we are continuing translating architecture." ${ }^{17}$ This translation from conventional representation cannot reproduce the experiential and phenomenological dimensions that are present when we experience architecture for ourselves.

The gap is scale. We are introduced to a new scale in any extension of ourselves through technology. ${ }^{18}$ Without scale, there is a fragmented sense of space and a fragmented sense of place in the translation of two-dimensions into three-dimensions or translating scale in one's mind. On the other hand, experience allows us to 'consider what it means to exist in space' and to 'consider how we are to exist it time'. Chinese geographer Yi-Fu Tuan defines experience as 'all the modes by which a person knows and constructs reality.' ${ }^{19}$ He further states that,

Experience is the totality of means by which we come to know the world: we know the world through sensation (feeling), perception, and conception.(388)

For Tuan, "Space is more abstract than place. What begins as undifferentiated space becomes place as we get to know it better and endow it with value", while, "Place incarnates the experiences and aspirations of a people" So, "The study of space, from the humanistic perspective, is thus the study of a people's spatial feelings and ideas in the stream of experience." ${ }^{20}$

\section{The realities in Architecture}

Drawing upon how space and place are conceived and defined spatially, the next step for architectural perception is to occupy the represented space in virtual reality. Changing the role of the viewer to the visitor, and then to the navigator, allows one to not only to see the [virtual] environment, but also to feel the space through percieved sensation. The interaction with space at the level of body (human scale) and the building mediates the digital and physical world, rendering digital space as digital place.

Virtual reality makes designed spaces more accessible to a wider audience, making the language of architectural projection accessible en masse as it contains the same perspectival logic inherent to our perception of the world. At the same time, engaging in the VR expereience also allows access to our visual experiences of the world to "reawaken individual eidetic memories in those who encounter our designs"21 grounded primarily, in the phenomenological and spiritual dimension of perceiving a space.

The question becomes, how can we implement virtual reality to mediate our perceptions of our designs from within, in order to comprehend the phenomenological and sublime qualities that the architecture can communicate through the method of experience? With qualitative appraoch of experience, can we design spaces that transform or transcend our expectations and/or ideas?

The VR interface hides its underlying complexity, while introducing a different way of engaging in represented space, by engaging in the possibilities of a 'poetic' translation, rather than a transcription of architectural representation. By combining visual awareness with sensation, the experiences of immediate presence within the environment create reciprocity between the digital and the individual.

VR is a tool which range from full immersion to limited mobility from a single vantage point in stereoscopic views. It is grounded in the premise of using the entire body to perceive by activating proprioception: the sense of the relative position of one's own parts of the body employed in movement relative to the digital space. ${ }^{22}$ Ultimately engaging, 'the unconscious perception of movement and spatial orientation arising from stimuli of one's 
body. ${ }^{123}$

The objectives in the following chapters are to identity

and challenge the experience that can be drawn out in virtual

reality as a medium through experienced design, and examine

the role of perception in architectural design. Experienced design

focuses on the quality of the user experience in tandem with

the design function in an attempt to draw from many sources

including theories of perception, storytelling, heuristics, design

thinking and virtual prototyping by referencing our 'reality', that

is not fixed and absolute, but expresses our ability to experience, visualize, articulate, and participate in the phenomenal reality of

the world.

1 According to Eco, the image is "....in want of 'more." Eco, Umberto. Travels in Hyper Reality: Essays. Harcourt Brace \& Company, 1990. p4 2 Dunning, William V. Changing Images of Pictorial Space: a History of Spatial Illusion in Painting. Syracuse University Press, 1991.

3 Pallasmaa, Juhani, The Eyes of the Skin: Architecture and the Senses, Wiley, 2014. p38 4 Carpo, Mario. The Second Digital Turn Design beyond Intelligence. The MIT Press, 2017. p101

5 ibid, p.96

$6 \quad$ ibid, p. 55

7 "Denise Scott Brown On the Past, Present and Future of VSBA's Groundbreaking Theories." ArchDaily, 7 Oct. 2016, www.archdaily.com/796821/ denise-scott-brown-on-the-past-present-andfuture-of-vsbas-groundbreaking-theories?ad_ medium=widget\&ad_name=interviews-articleshow.

8 Pallasmaa 33

9 ibid, Pallasmaa quotes Susan Sontag
10 Rae, M. A. (2017). Architecture and Theology The Art of Place. Baylor University Press. P.22 11 Beigl, Michael. "Disappearing Architecture: From Real to Virtual to Quantum". Springer Science \& Business Media. 2005.

12 Term used by Gomez to describe the process of the building practice, Pérez-Gómez Alberto, and Louise Pelletier, Architectural Representation and the Perspective Hinge (MIT Press: 2000), p.3

13 "Program as Horizon - Frank Weiner, Meditations on Two Competition Projects, 2012." E2A Architects Zurich, www.e2a.ch/news/programhorizon\#/page1/.

14 Morris, Mark. "Digital Space and Analog Place: A Shared Reality." The Value of Design: Design Is at the Core of What We Teach and Practice. ACSA Press, 2009.

15 Pallasmaa 34

16 Porter, Tom. "The Spatial Codes", The

Architects Eye: Visualization and Depiction of Space in Architecture. E \& FN Spon, 1997, p111.
17 Politakis, Charalampos. Architectural Colossi and the Human Body: Buildings and Metaphors. Routledge, 2018.

18 McLuhan warns that any extension of man is met by an extension of scale: "This is merely to say that personal and social consequences of any medium- that is of any extension of ourselvesresult from the new scale that is introduced into our affairs by each extension of ourselves, or by any new technology." McLuhan, Marshall. Understanding Media: The Extension of Man. Harmondsworth: Penguin, 1964.

19 Tuan, Yi-Fu. Space and Place: the Perspective of Experience, University of Minnesota Press, 2014, p387-422.

20 ibid, 388

21 Schaik, Leon Van. Spatial Intelligence New Futures for Architecture. Wiley, 2008. P.142

22 Proprioception, wikipedia

23 Proprioception definition; (In physiology: the neurological ability of the body to sense movement and position) 


\section{PART I: REPRESENTATION}

01 Verbal to Visual to Spatial (but tactile) perspective

02 Virtual Realities

Immersion, Application, and Virtual Prototyping 


\section{Verbal to Visual to Spatial (but tactile) perspective}

"It's not what you look at that matters, it's what you see."

-Henry David Thoreau

In the quest to understand to our world, perspective recognized a relatedness of our world to our visual field, creating a representational paradigm that transformed embodied reality into a visually accessible realm. Pallasmaa highlights how perspective aligned our view of the world:

The invention of perspectival representation made the eye the center point of the perceptual world as well as of the concept of the self. Perspectival representations itself turned into a symbolic form, one which not only describes but also conditions perception. (Pallasmaa 18)

Prior to the Renaissance period, the main vehicle of transferring data was not visual, but verbal. Perspective codified the way artists, scientists, and people saw and related to the world. For the first time, perspectival images could convert infinite distance in space into an arrangement of points and lines on a flat picture plane. Alberti encouraged painters to consider the frame of a painting as an open window. He cautioned that the idea is that perspective translates "to an almost infinite distance", whereas the plane onto which it is projected is not infinite. ${ }^{1}$ The process is comparable to an early stage of data compression. Alberti's technology can convert 3-D objects into planar notations, and equally reverse them-by a process known as photogrammetry. The process had limited practical interest at the time, with greater interest following the invention of photography. 


\subsection{Spiritual to visual}

Beyond data compression, the origin of perspective was rooted in the religious and moral preoccupations of the European late Middle Ages, "of the strongly held spiritual beliefs and assumptions of still devout Christian who longed for painted and sculpted images that could arouse the feeling of divine presence and reinforce their faith that God and his saints were still immanent in their daily lives"2 However, Alberti intended that his perspective was 'more important to the moral rather than the mythical message of Christian stories. ${ }^{3}$ Representation of biblical storytelling through the medium of stained glass demonstrates the value of the image- which could communicate to a larger audience, and displayed image as illustrative of God.

\subsection{Verbal to Visual}

The fixation of the viewer at the center of perception framed the visible world around the eye of the spectator. Geometric linear perspective became a new way to represent 'reality', influencing architectural thought and practice from that point on. ${ }^{4}$

The inability to easily reproduce images was due to the impracticality of artist purpose; Illustrations were uncommon, apart from the geometrical diagrams used in science and technology work. Works of art were often reproduced from verbal description. ${ }^{5}$ It is worth reproducing Carpo's quotation of 'the last encycolopedist of classic culture', Isidore of Seville, to connote the ancient mistrust of visual communication: 'images are always deceitful, never reliable, and never true to reality.' ${ }^{6}$ The lack of identical reproduction and identical 'reading' left copies to the mercy of talent and good will.

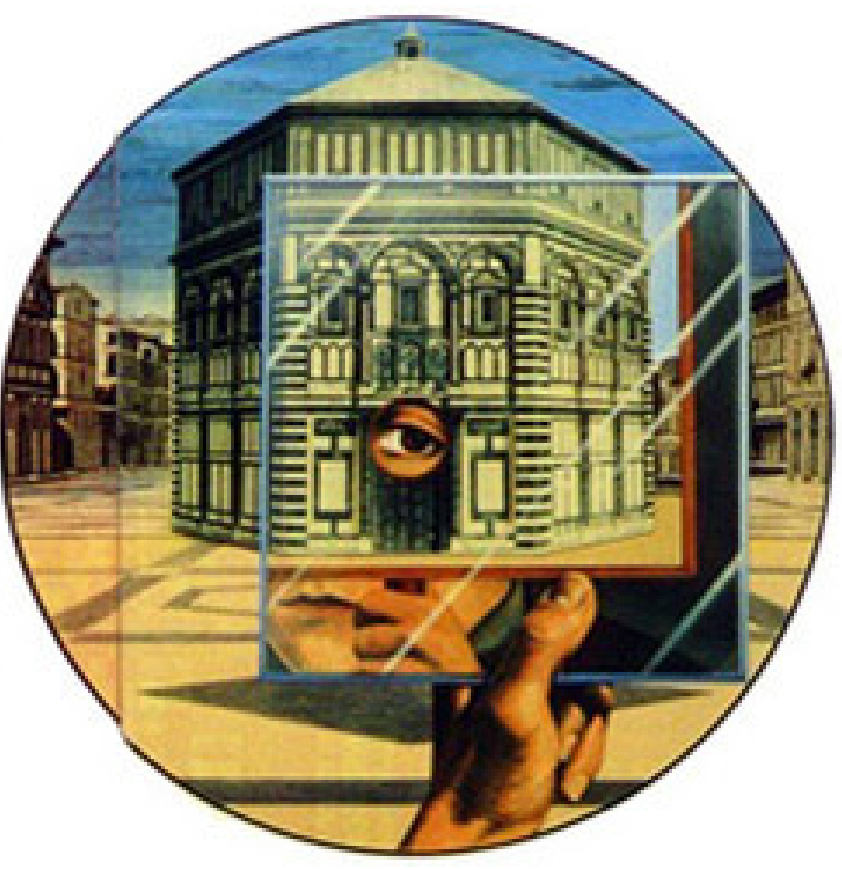

Figure 2.1 Mirror experiement 


\subsubsection{Ceci teura cela -This will kill that}

The printing press made information widely available, and translated bibles to many languages. As a result, there was a shift from oral to written speech, and prayer shifted from oral prayer to private reading. Printing allowed people to study religion without the need for a chapel, and 'the building became less essential in the religious order'. The printed word spurred the protestant reformation, and as a result, the generation-long conflict destroyed many buildings. The Christian era of the 15-century believed that architecture was 'the great book of humanity' and the principle expression of man. ${ }^{7}$ The fear was that the solid and durable 'book of stone' (otherwise known as architecture) was about to crumble to make way for the paper-book.

\subsection{Visual to spatial, but tactile to visual}

The newfound trust in images caused the eye to replace the hand in the hierarchy of the senses, in the primacy of painting over other forms of communication. Images became equal to the written word.

The Albertian definition of painting is that it is "an entirely mathematical construction". ${ }^{\text {P }}$ rojection was believed to be a form of 'science', so the final drawing demonstrates the laws of perspective, "because reality is what it is, but a perspectival drawing represents (in a sense, reenacts) reality through the laws of perspective, hence in a perspectival drawing all we see is 'measured."' Perspectival drawings are a form of "augmented reality", where they imbed the proportional measurements of what they show, as our eye cannot see in depth.

Our vision is based on two slightly different retinal images, and stereoscopy is based on "discrepancies between two monocular perspectival views" where the brain combines two 
images into one. Victorian inventor Charles Wheatstone discovered the process of binocular vision in 1838, "whereby human mind creates the most vivid belief of the solidity of an object". ${ }^{10}$

Two centuries after Alberti's invention, Galileo proclaimed planar projections as the only true notation of our cognitive experience of the physical world. The main message of perspective was its planarity. The notion of its cognitive translation of space revived in the 19th century. Wheatstone's invention of 'solid images' occurred roughly at the same time as analog photography and was overshadowed by its success. Wheatstone explains:

...our mind builds up solid volumes by interpreting two synchronic perspectival images taken from two slightly different vantage points, but in the absence of the second image, similar results can be obtained by comparing monocular images taken in a quick sequence from a moving vantage point. ${ }^{11}$

Among Alberti's inventions, Alberti recommended perspective to painters, and scaled drawings to architects. Gaspard Monge originally formalized parallel projection at the end of 18th century in an effort to link time and place with ideas and experience. Gomez ties projection to the space of representation defining it as 'the site of ontological continuity between universal ideas and specific thing.' 12

The set of orthographic projection derived from the techniques and methodology of Nicolas-Louis Durand in his Precis des Lecons d'Architecture 1802 and 1813. The Macanisme de la composition is the first design method to be dependent on the capacity of projection founded on descriptive geometry. The art of compressing large 3-D objects onto paper, is the result of a product of philosophical tradition and technological worldview of the 19th century in Europe. Orthographic projection continues to be a primary notational tool.
The scientific precision of Alberti's method to 're-enact reality' communicated design in its ability to compress and reconstruct data, as well as provided the designer with opportunity for feedback in construction. The design capabilities of Alberti's perspective theory promised the painter to 'make the same drawing, which he claimed could be reconstructed (by reversing the visible proportions and measurements with the technology).

In the 15th century two image-making technologies dramatically influenced culture: perspective and xylography, followed by rise of mechanical woodblock printing. The two technologies created a twofold trust in the image: the artist portrayed true nature, while the printer portrayed the true drawing. The Western (modern or contemporary) world became visual from that moment.13

The two-dimensional that is so deeply ingrained in Western culture, continues to battle for superiority with the threedimensional-mostly due to habit and convention, but also due to the design capabilities of each media in communication and feedback possibilities.

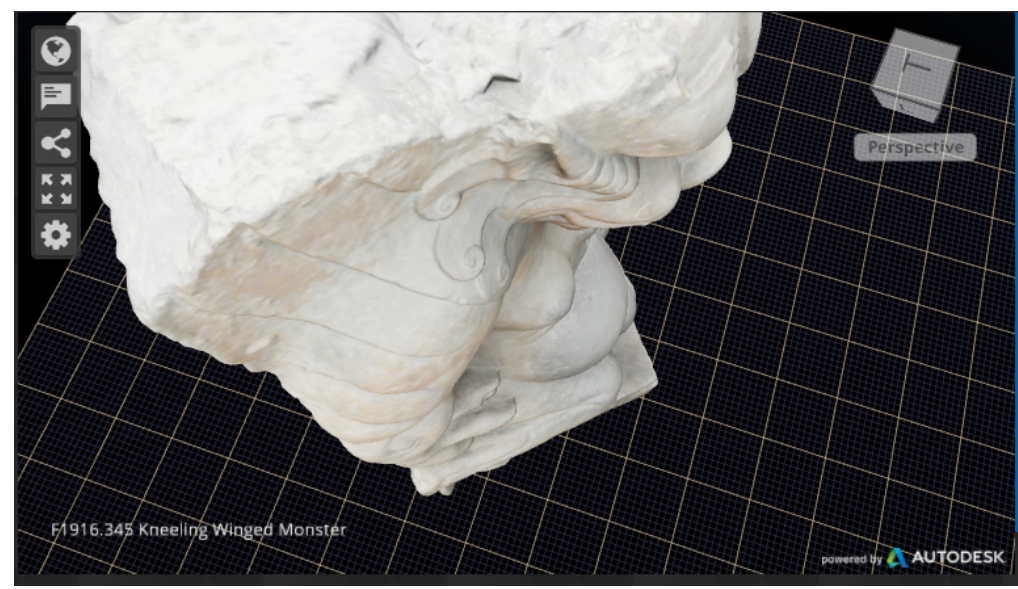

Figure 2.2 Smithsonian 3D scan, Kneeling Winged Monster. 
Today, the digital has the capacity to extract, sort and reveal so much more information. Threedimensional technologies can instantly record the spatial configuration of 3-D interior spaces and objects in spaces with use of $x-y-z$ coordinates. [a] Such as the ability to document incremental movement or changes of earthquake damage with GIS (geometric information systems) mapping with intention: using photometric surveys from aerial/elevated vantage points to interpret further movement [b] The Smithsonian Museum published a series of 3D scanned museum artifacts that allow users to pan and zoom each object on their screen. The digital artifacts scans reveal detail that is not possible to perceive from behind encased glass displays.

\section{ENDNOTES}

1 Carpo, Mario. The Second Digital Turn Design beyond Intelligence. The MIT Press, 2017, p101

2 Edgerton, Samuel Y. The Mirror, the Window, and the Telescope: How Renaissance Linear Perspective Changed Our Vision of the Universe, Cornell University Press, 2009.

3 ibid.

4 In the 15th century (1425), architect and engineer, Filippo Brunelleschi conducted his first optical/perspectival experiment at the Cathedral of Florence demonstrating that linear perspective could produce a realistic illusion of 3D space. Brunelleschi compared the perspective of his painting of the eastern façade of the Florentine
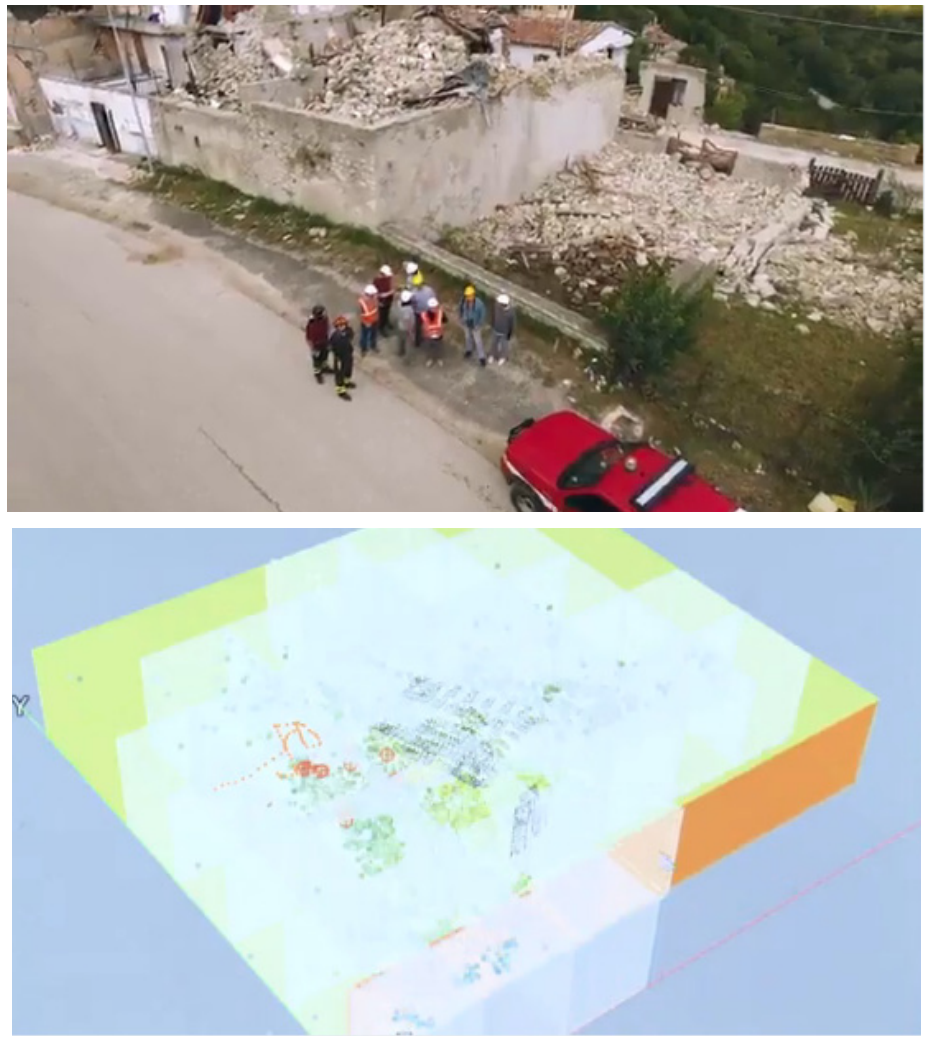

Figure 2.3 [ABOVE] Drones used to assess earthquake damage in Italy (2016) [Below] Digital scans are compared to detect movement
Baptistery with the built form with his constructed mirror in Florence. He translated the volumetric world onto a 2D surface by creating a one-point perspective of the baptistery with a small hole in the center. By looking through the hole (with the painting facing the building) to see the baptistery, and then by holding a mirror at arm's length in front of the painting, he sees it reflected back to him, making it easy to compare the baptistery and the painting's reflection. "Linear Perspective: Brunelleschi's Experiment.” Khan Academy.

$$
5
$$$$
\text { Carpo } 111
$$$$
6 \text { ibid, Carpo quotes Castiglione's }
$$

conclusions

7 Hugo, Victor, "Chapter Il: This Will Kill That."
The Hunchback of Notre Dame. 2015, http://www. online-literature.com/victor_hugo/hunchback_ notre_dame/24/

$8 \quad$ Carpo 111

9 Carpo 113-4

10 ibid, Carpo quotes Wheatstone 123, see Wheatstone, “Contributions. Part the First," p380.

11 Pérez-Gómez Alberto, and Louise Pelletier, Architectural Representation and the Perspective Hinge (MIT Press, 2000) p3.

12 Gomez 9

13 Carpo 114 
"Perhaps the essence of VR ultimately lies not in technology but in art, perhaps art of the highest order. Rather than control or escape or entertain or communicate, the ultimate promise of VR may be to transform, to redeem our awareness of reality-something that the highest art has attempted to do and something hinted at in the very label virtual reality, a label that has stuck, despite all objections, and that sums up a century of technological innovation. VR promises not a better vacuum cleaner or a more engrossing communications medium or even a friendlier computer interface. It promises the Holy Grail."

-Michael Heim, The Essence of $V R^{1}$

"Rather than [Mark] Weiser's dream of technologies of disappearance, the images propose, through wireless, a technology of many appearances."

- Luis Hernan and Martyn Dade-Robertson² 


\section{Virtual Realities: Immersion, Application, Virtual Prototyping}

All realities are virtual. We simultaneously create and perceive our world. - Jason Silva ${ }^{3}$

\section{From reality to virtuality}

Reality is a construction. As Joseph Campbell said, 'reality' is just a word and it has quotations marks around it. ${ }^{4}$ There is no such thing as objective reality. Reality includes the representation. Whereas, representation constructs reality, while perception and sensation show the kind of construction that happens.

VR is another kind of construct. We do not perceive reality as representation. Yet, we represent in order to participate in reality, and this applies to art and architecture throughout history. At the same time, new models of visualization technologies can almost instantly alter spatial perception in practice by varying the conditions of perception. It is worth remembering that reality is coupled to perception. "The conditions of perception can be varied within a broad range by a variety of technologies" $"$ by flattening our spatial awareness with screens.

The varying ranges of mixed, augmented, and virtual realities in current digital technologies have an overarching common philosophy driven to enrich the experiences of perception of the artificial-through interaction with the physical reality of human activity. ${ }^{?}$ Examining a qualitative approach in these new realities aligns with the human fundamental needs for visual information, that leads to question their integration and for which purposes they are best suited. It is valuable to note that we can also use these tools to enrich the experiences of newly designed spaces.

The new realities that have emerged in the field of architecture, such as augmented reality, mixed reality, augmented virtuality and virtual reality are all entering the architectural discipline promising (and rendering) possibilities for visualization and imagination, "likely to change the way we see almost everything, and represent and know the world around us." ${ }^{8}$ Human perception is in constant search for sensory stimulation, and we design with this in mind. 


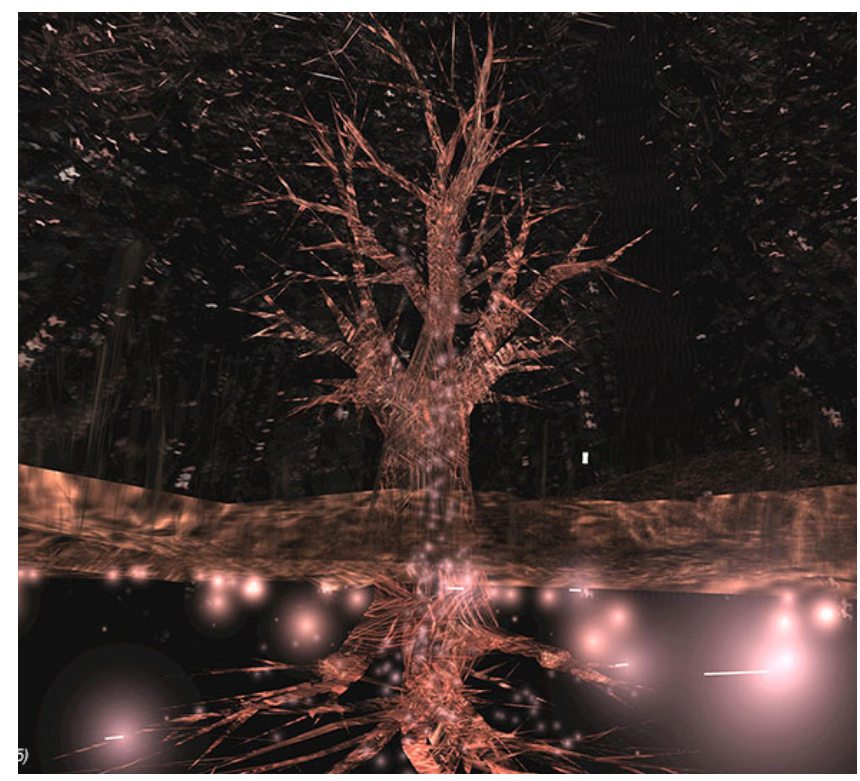

Figure 3.1 Osmose (1995) and Ephémère (1998) Virtual reality experiences by Char Davies which use the forest as a metaphor for human experience. "It's got nothing to do with representing nature. It's to help people awaken an aspect of themselves - another sensibility, a different quality of being." - Char Davies ${ }^{9}$

\subsection{The power of immersion}

"We do not see things 'as they are'-they are as we see them." - Schaik ${ }^{10}$

"Virtual space-as opposed to natural bodily space-contains the informational equivalent of things. Virtual space makes us feel as if we were dealing directly with physical or natural realities."-Michael Heim ${ }^{11}$

The term 'mimesis', is a writer's ability to pull the reader into a story, indicating how real or at least consistent with itself the story world is. ${ }^{12}$ Writers that choose to eternalize architecture teach us how to see through language and narrative. Within media, the action of immersion or 'being immersed', refers to an emotional or mental state, or 'a feeling of being involved in [an] experience' similar to the way a novel immerses a reader into an alternate 'story' world. It is the suspension of disbelief that makes the content seem real. The power of immersion can translate into the representation or imitation of the real world in art and literature. An example of experiential immersion is Char Davies's famous virtual reality piece Osmose (1995) and Ephémère (1998) premised on personal experience in the context of a simulated virtual forest. ${ }^{14}$ The virtual connection to nature idealized spiritual search and reflection using 'the artificial [to] regain a lost sense of open harmony with the natural.'13

Immersion is a metaphorical term derived from the physical experience of being submerged in water, which is comparable to the sensation of being surrounded by another reality as different as water is from air. The sensation takes over our attention and perception operations. ${ }^{15}$ In the architectural context, a diegetic level of immersion is the ability to immerse one in the spatial environment as part of the narrative, without breaking the fourth wall.

In virtual reality, successful absorption into the media content is called "presence", allowing the user to be 'in it' like a good book. Presence is related to the phenomenon of distal attribution or externalization, which references our perceptions beyond the limits of our sensory organs, and beyond our immediate physical surroundings. When perception is mediated by technology, we simultaneously perceive two environments, the environment actually present, and the environment via the medium. The experience of an environment through a communication medium is defined as telepresence. ${ }^{16}$ Virtual reality uses the perception of depth and binocular vision to create stereoscopic display. The creation of perceived virtual environments are created by two slightly different angles simulating depth. ${ }^{17}$ The simulation of physicality in the form of 
texture mapping and surface rendering is of high priority in VR.

Virtual reality is a tool for increased immersion and an insight to Experience - as the participatory nature of ritual. When you can feel the weight of the present moment with the viewer at the deictic center. ${ }^{18}$ The user's perception is no longer fragmented, but instead can be examined in an immersive wholepicture render to experience the sensory qualities of the space. VR allows us the application of the phenomenon, enabling designers and architecture storytellers not to change their process, but implement a new language of scale to experience in design.

\subsection{Emergence of VR applications}

The shift to digital technology created an apprehension of the collapse of the world into cyberspace. Just like the translation of infinite space to a physical plane, digital technologies suggest infinity. On the contrary, cyberspace is often treated as a physical location. ${ }^{19}$ This may explain why we use language such as, "He is here with us," to denote the virtual presence of a person, suggests that the words here and there are suggestive of presence. Webster's New Universal Unabridged Dictionary (1989) defines virtual as "being in essence or effect, but not in fact", more specifically, "being on or simulated on a computer or computer network". ${ }^{20}$ Virtual derives from the Latin, virtus, 'virtue' or in the sense possessing certain virtues. ${ }^{21}$ Nevertheless, the real interest lies in what it can do.

The focus of VR was originally technological, rather than experiential. The term, virtual reality, coined by Jaron Lanier (chief executive officer of VPL Research Inc., manufacturer of VR products and equipment) in $1989^{22}$, is premised on the perception of depth and binocular vision to simulate a stereoscopic field of view in what could be better understood as a 'visual', rather than 'virtual', reality simulation. In the early 1990s, researchers at MIT used the term 'virtual environments', to avoid the phrase 'virtual reality' that was believed promised the concept of artificial intelligence. Today, virtual environment is used interchangeably with virtual world and virtual reality, as an ambiguous term used to define an interface for a participant to experience a computer generated first person perspective. However to inhabit cyberspace is not uncharted territory. Virtual reality [VR] is not a new phenomenon, what is new, is its commercialization. Since its inception prior to the Second World War, virtual reality has become increasingly affordable, and therefore largely accessible within the last decade. ${ }^{23}$

German philosopher Thomas Metzinger's technological metaphor of conciseness places our physical 'reality' as a virtual inner reality that simulates us in the world, embedded in a 'scene':23

Nature's virtual reality is a conscious experience - a real-time world-model that can be viewed as a permanently running online simulation, allowing organisms to act and interact.

He contends that nature's virtual reality has achieved what we are striving for: "the phenomenal properties of presence and full immersion". The ideal virtual reality will interact and react to its user. Metzinger defines this medium as virtual interface that will continuously learn from its envrionment:

A [virtual interface] creates an interactive medium that conveys information to the user's senses while constantly monitoring the user's behavior and implying it to update and manipulate the virtual environment. (104) 


\subsection{Representing and reproducing the three dimensional}

Similar to the way Alberti approached paintings as windows to the world, modern-day ]virtual windows' give us access to the world of fantasy. Augmented reality computing platforms by bringing augmented reality to phones. One example is Project Tango by Google, which is a motion sensing and depth sensing 3-D model of interior space through the natural movements of someone waking through it. The intention is to give mobile devices a human-scale understanding of space and motion. The project began from the problem of interior navigation, localization and mapping, developing navigation for the visually impaired at a prototype stage. Alas, a new project development called ARCore replaced Project Tango as its less powerful but more accessible (aligned with the technological push for the 'visual search' option), with the added advantage of portability and accessibility. ${ }^{24}$

In 2012, the first commercial light field camera came to market. The picture of the portable camera isn't projected onto a screen, but creates a 3-D model in space that can be later revisited by, 'rotating the central ray and changing the point of view.. ${ }^{25}$ The vantage point of the user may be fixed or movable with a wide angle of head rotation, with full control of focus after the pciture has been taken.
Translational Movement in Three Perpendicular Axes

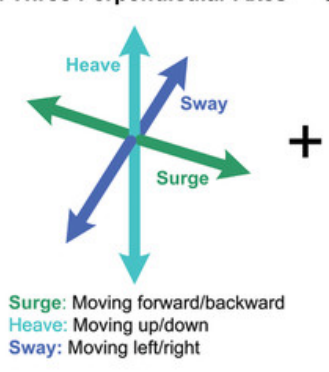

Rotational Movement about Three Perpendicular Axes

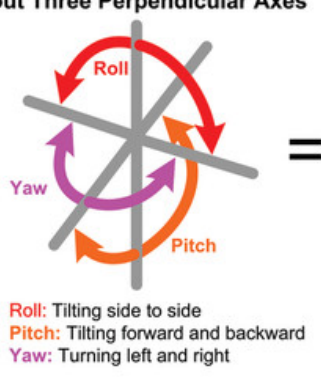

Six Degrees of Freedom

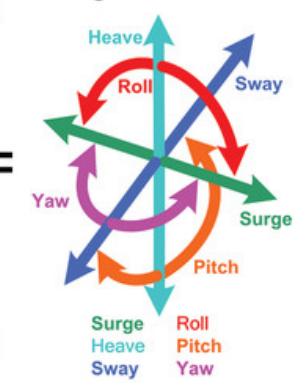

Figure 3.2 Degrees of freedom 


\subsubsection{Degrees of Freedom}

The limitations of the technology either render the content as a 360 video limited to 3 degrees of freedom or enable 6 degrees of freedom but be limited by the graphic capabilities of a real time engine.

The Lytro Volume Tracer ${ }^{26}$ promised the highest sense of immersion in VR content using light fields as the basis to take a 3-D model and create 2-D renders that 'contain complete light field volume for playback". ${ }^{27}$ This enables 6 degrees of freedom and retains the quality of a fully rendered, retraced environment, said to create the highest sense of immersion, and the highest fidelity content. By creating a new reality or another level for quality for entertainment and media, the designer can tell the story while feeling the dimensions and scale of a space before it has been build. This is an example of visual conception that allows one to perceive new products at a high level of fidelity.

There are head-mounted displays for augmented and mixed reality. Common problems in 6 degree display are the screen door effect (appearance of grid over display), latency (delay in action and reaction), and motion sickness (caused by visual stimuli). The competitive edge is physical lightness (over data lightness) of images over models. Images are physically lighter than 3-D models and are able to compress a lot of information. Now data is endlessly available, accessible and easily affordable to copy. ${ }^{28}$

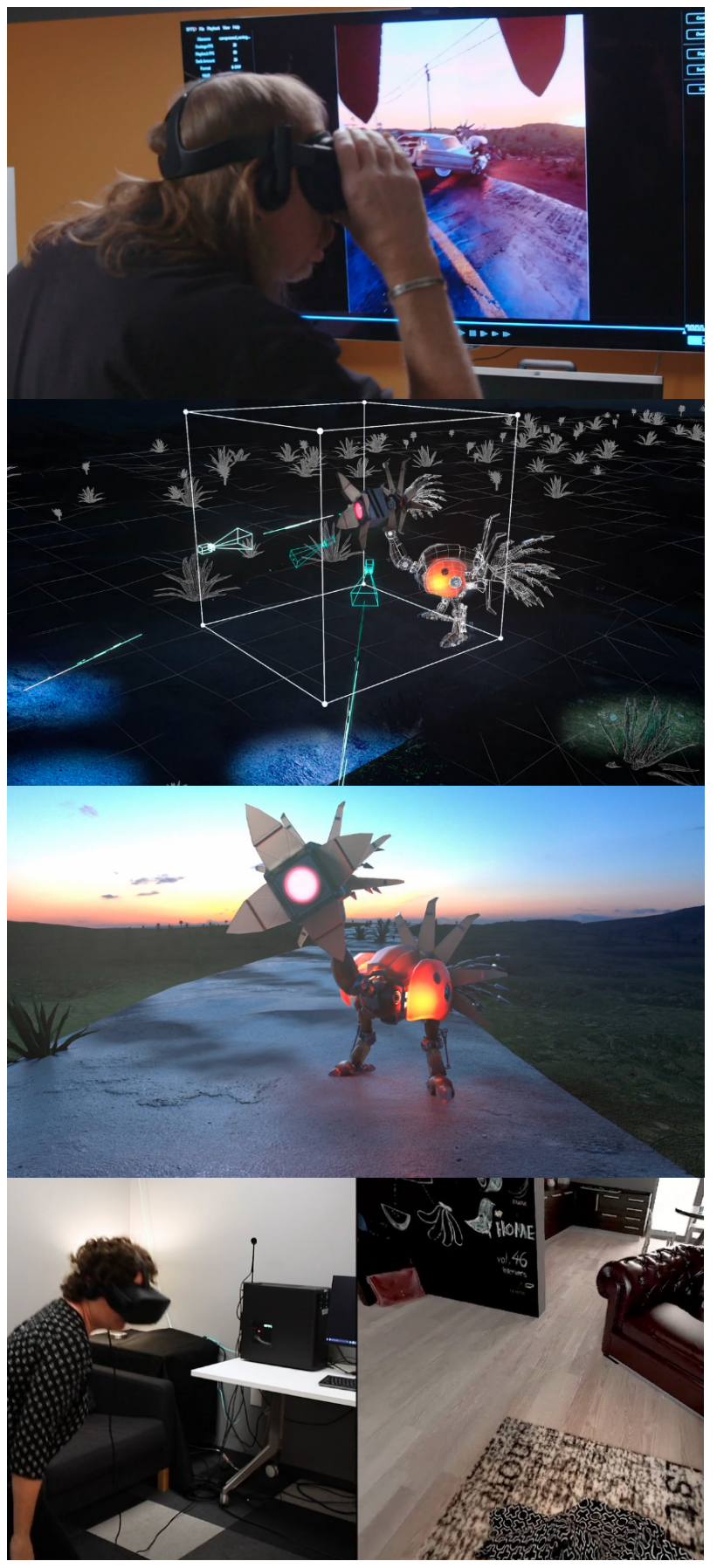

Figure 3.3 Lytro Volume Tracer 


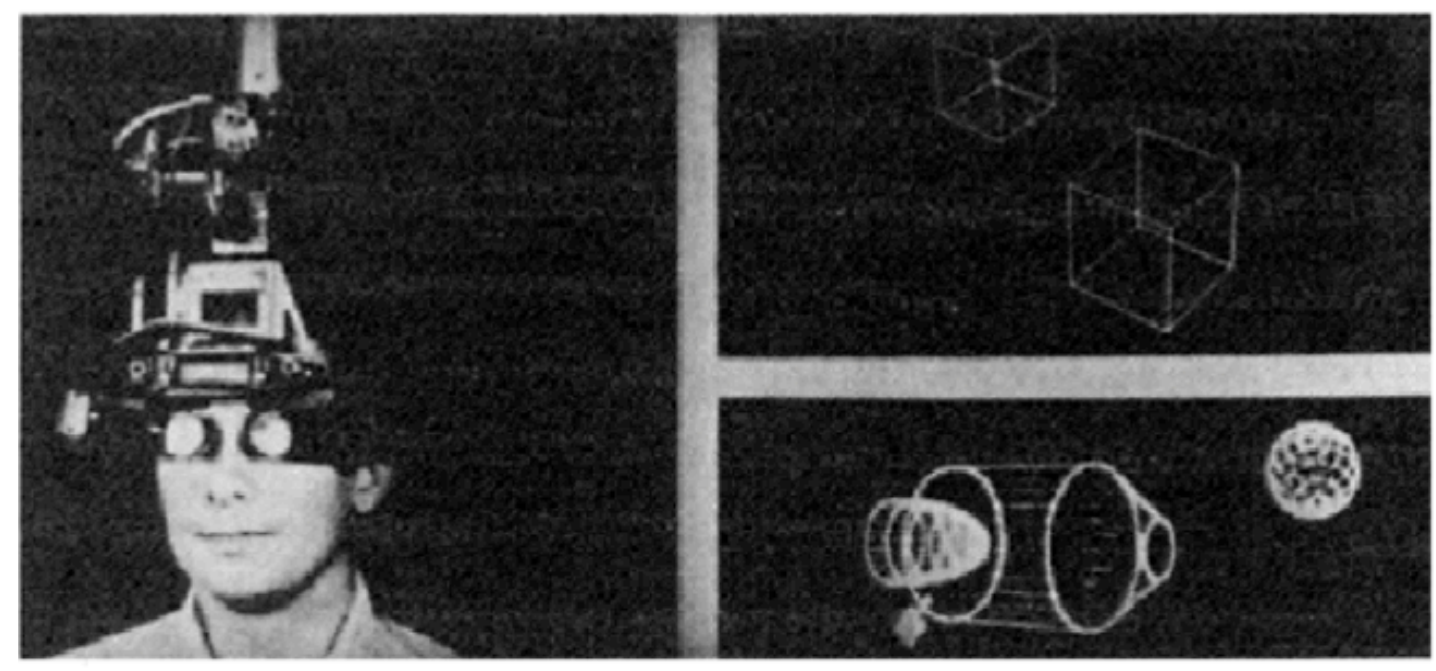

Figure 3.4 First computer-driven, head-mounted viewing device developed by Lvan Sutherland to give the viewer the illusion of actually being in the syntheic world defined by the computer. 
Figure 3.5 Virtual Reality in industrial applications

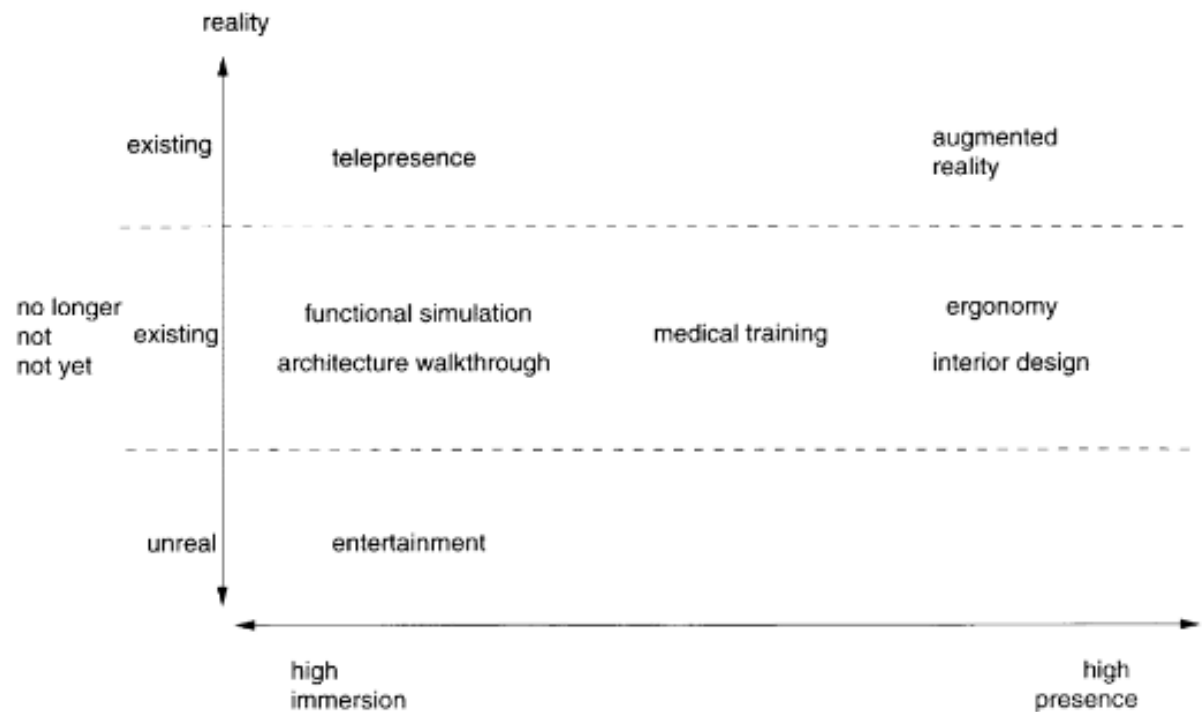

\subsection{Virtual Prototyping}

An artificial reality perceives a participants' action in terms of the body's relationship to a graphic world and generates responses that maintain the illusion that his or her actions are taking place within a world. ${ }^{29}$

\section{VR bridges the gap between the incomplete} (fragmented) image and the projected reading of the ideas, desires and personality of the perceiver. The 'push to complete the image' or the 'what is left to the imagined' is reduced. Physical or computed models become visually tangible, with the ability to navigate the view by head movement and push the boundaries of the medium to play with immersive scale. Architectural walk through(s) are currently used as part show-part evaluation-to elicit constructive criticism from clients and users not familiar with orthographic language. "The power of VR lies in its direct appeal to the senses." ${ }^{30}$

Common aversions of virtual reality are cyber sickness or motion sickness that is caused by not meeting an acceptable frame rate, FOV, or latency (i.e. anything over 20 milliseconds not fast enough). Human FOV (field of view) is 180 degree-270 degrees with eye movement. In comparison, Vive and Rift have 110-degree view, Cardboard has 90-degrees, GearVR has 96, and Daydream is rumored to have 120 degree display. ${ }^{31}$

Simulated environments can be disappointing by appearing 'dead and empty' or "devoid of life that architects readily suggest in conventional media". 32 However, immersion provides impressive sensations, especially when rendered in high quality. A poor render will appear poor in immersion. A sensory stimulated render will appear to capture the viewer.

The participatory quality of VR provides a new layer of information, as it is now able to collect immediate responses on an array of parameters including acoustics, lighting, materials, and reactions through 360-degree whole-picture immersion mockups. The data interpretation enables a new level of testing and altering virtual prototypes. Architects can 'check their design' and walk 


\begin{tabular}{|c|c|}
\hline & Most Common \\
\hline Concept & $\begin{array}{l}\text { VR: Used as a design tool } \\
\text { RP: Used for sketch design communication }\end{array}$ \\
\hline Established Design & PR: Client visualization \\
\hline & VR: Client visualization $\quad R P$ and 3D scanning: Design verification \\
\hline Detailing & \multirow{3}{*}{$\begin{array}{l}\text { VR: Coordination and design resolution } \\
\text { RP: Coordination and design resolution }\end{array}$} \\
\hline Production & \\
\hline Assembly & \\
\hline
\end{tabular}

Figure 3.6 Uses of Prototyping in construction design

clients through their projects, but the real benefit is the ability to 'self-curate' and navigate an experience in space. Architect can determine how the 360-degree experience is different from what will be reality.

Head mounted display range from mobile-dependent to movement tracking systems such as Oculus rift. Virtual prototypes can simulate products, buildings and urban settings as well as digital representations of design proposals for 'explorations, analysis and evaluation' with the intention to be physically realized.

A prototype is "a partial, approximate, or abstracted realization of a component or system that is constructed before the real thing in order to advance the design process".33 The goal is to "provide information for further refinement and development of a design; to identify design errors or potential failure points; to provide a basis for choosing among options or deciding whether to proceed to the next stage; and to persuade decision makers." ${ }^{.34}$
Experimental prototype that can be 'tested', are rendered 'successful' at the minimum expense of time and resources. Architects can check their design and walk clients through their projects by connecting virtual worlds to design prototypes. A quick maquette may provide rough guidelines, versus a detailed and costly prototype during construction stages. The information gained is of high value for design failure, safety, and unforeseen or unnoticed conditions.

Virtual prototyping is the "exploitation of simulation processes for the test, evaluation and modification of prototypes in virtual design environments." ${ }^{35}$ Advantages are that it is less expensive than the physical equivalent. Digital models that serve as design and later construction documentation can also serve as a visualization tool that is easy to edit and make design changes, as it is built with variation, exploration and testing in mind, such as performance simulation tools for lighting, structural, material, etc. Virtual and physical prototypes provide different information as to what can be interpreted for feedback: 


\section{Information Conveyed ${ }^{36}$}

Rapid/Physical Prototyping (RP): Geometry and Form Virtual Prototypingv (VR): Look and Feel

For design visualization, VR has the ability to covey the environment that will be created within various perspectives and different levels of abstraction, creating the opportunity to: make extensive decisions regarding the functionality of room design; use VR mock-ups for design review due the 'enhanced capacity for an immersive experience'; substitute full-scale physical mock-ups that are known as the truest representation of the design (PMUs are a common practice in the construction phase of healthcare facilities) limited to minor space modifications to "Implement their own "physical" and "biological" laws". Communications between designers, constructors and owners become easier and ultimately, virtual prototype testing exercises the ability to:

[1] Expand the range of design processes to 'exercise design judgment'37

[2] Economically simulate conditions ${ }^{38}$

[3] Observe the impressions created by newly designed space

The attraction of virtual prototyping is tied to the already existing digital, design documentation and 3-D modeling used to represent architectural ideas. The idea of integrated computer-aided design that has been around since the 70 s is to 'build digital models that support documentation and visualization, engineering analysis, and various forms of performance simulation." ${ }^{39}$ Using immersion to make design decisions is anchored in qualitative research, that allows edits and changes, exploration of various options (i.e parametric modeling variations), and development of analysis and simulation software to test lighting or acoustic simulation.

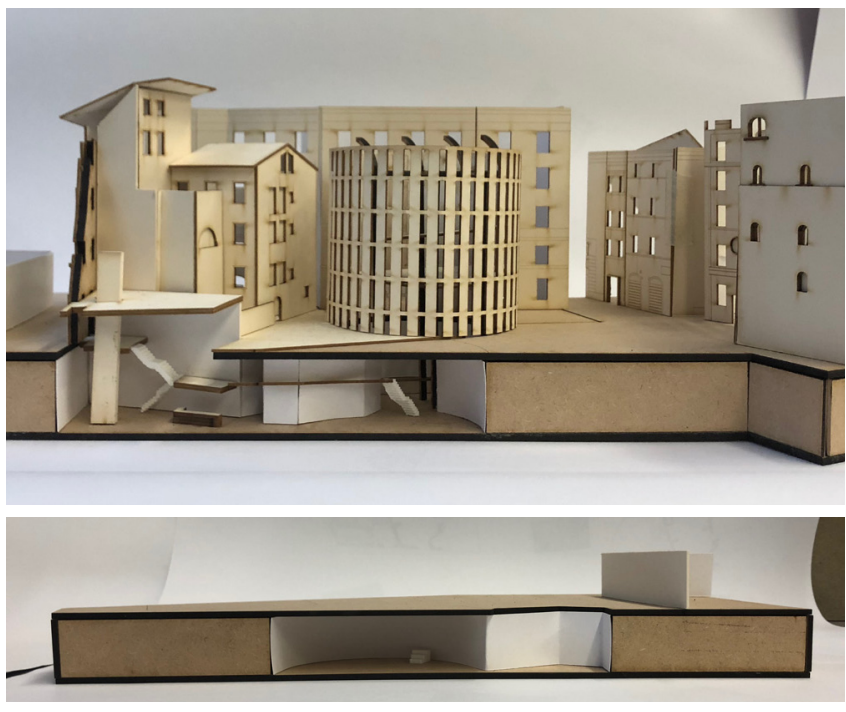

Figure 3.7 Physical model at 1:200

Virtual reality's self-referential system creates a sense of spatial reality within virtual environments. Relevant in-situ context impacts a design decision when seen in VR versus when looking at orthographic drawings or renderings in how the concept compliment 'reality'. Ultimaelty, the model can be adapted and the process repeated and evaluated again.

On the principles of cyberspace, Michael Benedikt examined the laws of nature that should be retained in cyberspace as fixed conditions of all phenomena. ${ }^{40}$ These principles help retain the qualities of physical world as the laws for virtual objects and subjects mirror the 'physical, social, economic and ideological world of the 'real'. Examining architectural metaphors, Benedikt defines the laws for objects and humans in the virtual world: "the rhetoric of virtually, is it denies the gap between representation and reality, insinuating a "presence" and naturalness where in fact only mediation exists." ${ }^{41}$

That gap is further underlined by Michael Heim, as known as the 'philosopher of cyberspace', who claims that no image can convey the $3 \mathrm{~d}$ optical experience of the space. ${ }^{42}$ 


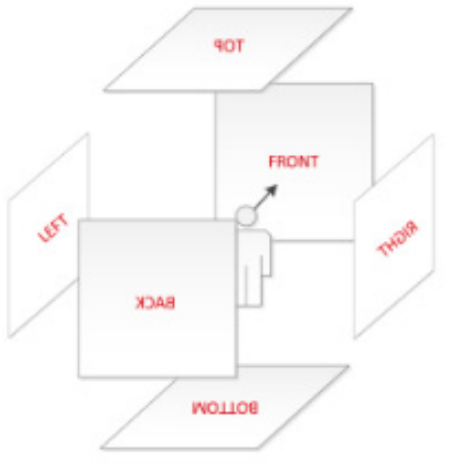

Figure 3.8 Cubemap of 12 view renders (stereo display)

"Associating seeing with being has significantly shaped the development of Western thinking... With the eye an extension of the mind, and the mind divorced from the body, the actuality of sight has been transformed into an ideology: the individual becomes the center of its world, always looking outward with its gaze-god like-scanning, naming, and colonizing the universe." (33)

However, a 360-streoscophic image can create a sensation of being elsewhere, providing the ability to observe an impression of space and place.

The design research in the following chapters will explore perception of designed space from various stationary positions where the user is physically at rest with a vantage point of $4 \mathrm{ft}$ when seated and 5 and a half when walking, creating 18 to 24 inch difference as, "basic viewing orientation is almost gyroscopically aligned to the horizon." ${ }^{\prime 3}$ We unconsciously search for the horizon.

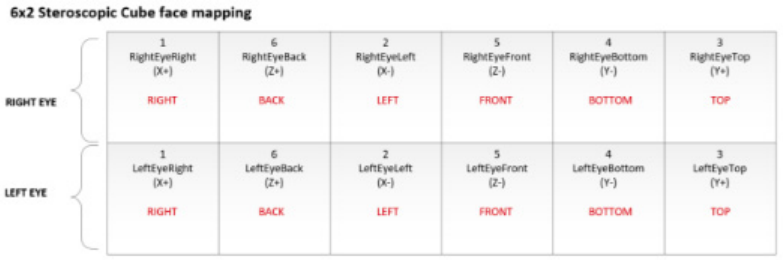

Figure 3.9 Cubemap order of 12 view renders (stereo display)

Our 'horizon seeing nature' moves with us, when we ascend or descend, orientated partly due to the constraints of gravity.

The design research will use Fixed Point Renders to perceive digital place through [1] perception from one station point, [2] exploration made by eye movement. FPR, or fixed point render, is a term used by Yulio ${ }^{44}$ to describe a stereoscopic cube map generated from a fixed camera position in an environment made up of 12 images. ${ }^{45}$ Combining multiple FPRs together can create a VRE: a virtual reality experience, ${ }^{46}$ allowing the user to teleport from on scene to another.

\subsection{Proprioceptive feedback vs. visual information}

Proprioception is a term that defines the sense of the body and its parts spatially; within peripheral sensation, creating a fundamental sense of self. While the promise of immersive virtual environments 


\begin{tabular}{|l|l|}
\hline Domain & Example Applications \\
\hline $\begin{array}{l}\text { "Being There", } \\
\text { experience for the sake } \\
\text { of experience }\end{array}$ & $\begin{array}{l}\text { Phobia therapy: [Rothbaum 1995] } \\
\text { Aesthetics: [Davies 1996] } \\
\text { Entertainment: [Pausch 1996] }\end{array}$ \\
\hline $\begin{array}{l}\text { Training and practice of } \\
\text { different skills }\end{array}$ & $\begin{array}{l}\text { Surgery: [Hunter 1993] } \\
\text { Military : [Macedonia 1994] } \\
\end{array}$ \\
$\begin{array}{l}\text { Maintenance: [Wilson 1995] } \\
\text { Wayfinding: [Witmer 1995] }\end{array}$ \\
\hline $\begin{array}{l}\text { Visualization of } \\
\text { unrealized or unseeable } \\
\text { objects }\end{array}$ & $\begin{array}{l}\text { Architecture: [Brooks 1986] } \\
\text { Fluid Flow: [Bryson 1992] } \\
\text { Nano-surfaces: [Taylor 1993] }\end{array}$ \\
\hline Design & $\begin{array}{l}\text { 3D models: [Butterworth 1992] } \\
\text { Cityscapes: [Mapes 1995] }\end{array}$ \\
\hline
\end{tabular}

Figure 3.10 Virtual Application Domain, 2001

suggests that we can perceive and interact with three-dimensional virtual objects, the underlying belief is to streamline a more natural and effective interactive interface between humans and machines. Defining the current key successful virtual-world application domains are: experience for the sake of experience, training, visualization of the unrealized, and design purposes (Table 1, Mine, r. M., page 1). Three-dimensional simulation in virtual environments has a natural application in the architectural disciple, as it involves the design of buildings prior to their realization. The profession has a long-standing tradition of two-dimensional plans, sections, and renders. Increasing complexity and brainstorming, requiring a multi-dimensional medium/media of representation introduces a need for physical modeling and three-dimensional computer modeling. Tools currently used include rendering, animation, panoramic views, interactivity (between user and architectural representation/architectural communication of space) and sense of immersion are still not readily integrated.

Researchers from the Universities of North Carolina and Berkeley have presented a framework for interaction and manipulation in virtual reality based on proprioception. ${ }^{47}$ The framework is structured around a desire for proprioceptive feedback over primary visual information, to provide further effective bodyrelative interaction (as concluded based on user observations). They observed that users routinely altered between framing closeup, detailed views (local) and pulled-back, full picture (global) views in the interactive system, resulting in the development of a 'headbutt zoom' (for CAD, molecular map tracing, technical illustration and preparation). Similar to conventional window displays, the user selects the view by positioning the hands like a frame, and switching between the two views by leaning forward and back. A step forward secures the chosen view. 


\subsection{The gap}

VR bridges the gap between the incomplete (fragmented) image and the projected reading of the ideas, desires and personality of the perceiver. The 'push to complete the image' or the 'what is left to the imagined' is reduced.

"Vision revels what the touch already knows. We could think of the sense of touch as the unconscious of vision. Our eyes stroke different surfaces, contours and edges, and the unconscious tactile sensation determines the agreeableness or unpleasantness of the experience. The distant and the near are experienced with the same intensity, and they merge into one coherent experience..." (Pallasma 46)

The representation of reality is virtually tied to capturing 'real-life appeal'. Great care and devotion is placed towards portraying the virtual world as close as possible to physical 'reality'. Additionally, there is an increased, immersive pleasure of enhanced perception through high fidelity and resolution. The advantages of VR include the following:

\section{- $\quad$ Fidelity, as mentioned above}

- $\quad$ Portability and availability

- $\quad$ Primary purpose of virtual reality is its mediating role in the participatory, which enhances our ability to participate in phenomenal reality

- A self-referential system: A stationary/movable vantage point allows the end user to choose what to look at. There is no layer of subjectivity as is seen in perspectival/projected imagery. As psychologist Willaim James claimed, your experience becomes what you attend to.

- We search for experience - that kind of human curiosity in form of traveling, seeking and exploring. We search for it in our imagination and through storytelling. Attention has become the daily currency, and VR allows the viwer to engage with what they chose

- $\quad$ From a commercial standpoint, to see something in use sells, because a great experience or a great narrative connects with people

- It is necessary to experience VR to understand

The relationship between information and its representation in a post-digital era exemplifies "...accounts of digital technologies which propose material-less disembodied interactions with computer-based information." (Dade-Robertson xi) In order to translate a more accessible user identity to the built realm, an intuitive mode of thinking and organization is required. The tools that inform architecture analysis are form, function and space. ${ }^{48}$ By experimenting with modeling, prototypes and simulations not to define the parameters of operation, but to enrich current methodology to experience, evaluate and comprehend the repercussions of design decisions.

The virtual and the physical can operate in parallel, to present a reconstruction of something no longer in existence or that has been demolished, such as Rome at the time of Julius Caesar or something proposed but never made physical, such as the work of Piranesi and archigram. It can serve as a utopia-an 'improved' or 'promise' version of the physical, or it can present a fictional, imagined space. The value of the mediated experience is seen by the qualities that emerge in our perception. 


\section{ENDNOTES}

1 Heim, Michael, The Essence of VR. (New York: Oxford Press) 1993, p 109-128.

2 Hernan and Dade-Roberstson reference Mark Weiser, also known as the father of ubiquitous computing. (Hernan, Luis, and Dade-Robertson, Martyn, Atmospheres of digital technology: wireless spectres and ghosts outside the machine, 2016, p.214-233.)

3 Silva, Jason.

4 Slattery, R. Diana. "Technology Mergers" Exploring the Edge Realms of Consciousness: Liminal Zones, Psychic Science, and the Hidden Dimensions of the Mind. Evolver Editions, 2012. P.328

5 ibid

6 Wang, Xiangyu, and Marc Aurel, Schnabel, Mixed Reality in Architecture, Design and Construction, Springer, 2010.

\section{$7 \quad$ Carpo 122 \\ $8 \quad$ Schaik 176}

$9 \quad$ Heim 33

10 Sherman, William R. Understanding Virtual Reality: Interface, Application, and Design. (Morgan Kaufmann, 2017), p8

11 Sherman 8

12 Heim, 1993

13 Janet Murray defines immersion

14 Steuer defines presence, quoted by Alison McMahan, see Steuer, Jonathan. Defining Virtual Reality: Dimensions Determining Telepresence. Journal of Communication; ABI/INFORM Global, vol. 42, no. 4, 1992, p73

15 Contains parallax: farther objects seem to move slower.

16 Michael Heim presents seven concepts of guiding VR by pioneers: Simulation, Interaction, Artificiality, Immersion, Telepresence, Full Body Immersion, Networked communications. Warns of VR plagues: Activity/passivity, manipulation/ receptivity, remote presence, augmented reality. "Heim: Essence of VR." The United States War on Drugs, web.stanford.edu/class/history34q/readings/ Michael_Heim/HeimEssenceVR.html.

17 Sherman and Craig 6

18 ibid, 8, Webster's New Universal

Unabridged Dictionary (1989)

19 ibid

$20 \quad$ Krueger, 1991

21 Palmer Luckey developed oculus rift; Facebook bought Oculus in 2014; Start up costs are low; Oculus Rift is about $600 \$$ and HTC Vive is at $800 \$$ (plus VR setup; which should be more than $1000 \$)$

22 Metzinger, Thomas. The Ego Tunnel. Basic Books, 2009. p104

$23 \quad$ Heim 33

24 Kastrenakes, Jacob. "Google's Project

Tango Is Shutting down Because ARCore Is Already Here." The Verge, The Verge, 15 Dec. 2017, www. theverge.com/2017/12/15/16782556/project-tangogoogle-shutting-down-arcore-augmented-reality.

25 Carpo 103

26 Accessed February 17, 2019 https://www.

lytro.com/volumetracer\#video

27 ibid

28 Lytro Support, www.lytro.com/

volumetracer\#video.

29 Carpo 103

30 Sherman and Craig 16

31 Delft 2001

32 Mullis, Alex. "How Does Virtual Reality

Work?" Android Authority, 15 July 2016, www. androidauthority.com/virtual-reality-work-702049/. 33 Mine R.M., Brooks, F., Sequin, H.C., Moving Objects in Space: Exploiting Proprioception in In Virtual Environment Interaction, University of North Carolina at Chapel Hill, University of Berkeley, 2001. 34 Mitchell, J. William. "Foreword- Virtual world, virtual prototypes and design" Virtual Futures for Design, Construction and Procurement. 2018. p xv

35 ibid, xvi

36 Oxman, Rivka. "Emerging paradigms and models in digital design- Performance-based architectural design.” Virtual Futures for Design, Construction and Procurement. 2018. p 3

37 Brandon, S. Peter, Kocaturk, Tuba. “Design, Engineering and manufacturing challenges", Virtual Futures for Design, Construction and Procurement, 2008, p. 36

38 ibid

39 ibid, p.36

$40 \quad$ Michell xxvi

41 Steinicke, Frank. Being Really Virtual Immersive Natives and the Future of Virtual Reality. Springer Berlin Heidelberg, 2016. P146

42 Dyson, Frances. "'Space', 'Being' and other Fiction in the Domain of the Virtual." The Virtual Dimension (1998) 27-45 (p.35-36)

43 ibid

44 Orr, Frank. Scale in Architecture. Chapman \& Hall, 1985. p23

$45 \quad$ Virtual Reality rendering platform

46 "Fixed Point Render Requirements." Yulio Support Centre, yulio.zendesk.com/hc/en-us/ articles/219131167; "Cubemap and Stereoscopic Images.” Yulio Support Centre, yulio.zendesk. com/hc/en-us/articles/218813758-Cubemap-andStereoscopic-Images.

47 "What Is an FPR?" Yulio Support Centre, yulio.zendesk.com/hc/en-us/articles/217173258What-is-an-FPR-.

48 Boff 1986

49 Dade-Robertson xi

$50 \quad$ Markus 12 


\section{PART II: PERCEPTION}

03 Sensory and Information-based theories of perception

04 The architectonic, the narrative, and the poetic

05 Functions across scale 


\section{Sensory and Information- based theories of perception}

"We plunge forward into the field of fresh experience with the beliefs our ancestors and we have made already; these determine what we notice; what we notice determines what we do; what we do again determines what we experience; so from one thing to another, altho the stubborn fact remains that there is a sensible flux, what is true of it seems from first to last to be largely a matter of our own creation." -William James 1907:112 1

\section{Why do we examine perception?}

In the fields of philosophy, psychology and cognitive sciences, "perception is the process of attainting awareness or understanding of sensory information". ${ }^{2}$ From the latin perceptio, perception translates to receiving, collecting, taking possession, or apprehension with the mind or the senses. Our perceptions emerge within a larger framework by which we view the world, facilitating understanding of situations. Human beings have a biological need to understand the nature and structure of the immediate environment, rooted in a Darwinian drive for survival and security. Early pioneers in the field of perception, William James and Ewald Hering, "address[ed] the real problems of man in relation to his total environment" and examined the how and the why we perceive the world around us. "...Perceiving is an act, not a response, an act of attention, not a triggered impression, an achievement, not a reflex."

William Ittelson, author of Environment and Cognition, produced three contributions to recent perceptual theory. First, action greatly impacts the information we obtain from the environment. Second, we seek information for a purpose. And third, there is a difference between object and environmental perception, which highlight several architectural issues. ${ }^{4} \mathrm{v}$ 
There are a several factors to how we perceive: ${ }^{5}$

[1] What we perceive to be real, what we see, is a reconstruction in our minds, a simplified model of the world, limited by our biology and physiology.

[2] Perception, including vision, is an active process. It requires action and integration
[3] The actions that we take, affects the reality and the meaning that we construct in our mind

[4] Perhaps most importantly, even when presented with the same information, the same images, everybody will experience-will see-something unique and personal, which nobody else can see or maybe even understand.
Figure 4.1 Spotlight model. In the spotlight model, the focus is the central area of attention, pertaining to highly detailed information. The fringe contains the less detailed information, and the margin is the cutoff for any information perception.

Figure 4.2 (1) Theme: data at the focus of attention organized according to Gestalt law. (2) Thematic field: unattended data relevant to the theme. (3) Marginal consciousness: unattended data not relevant to the theme.

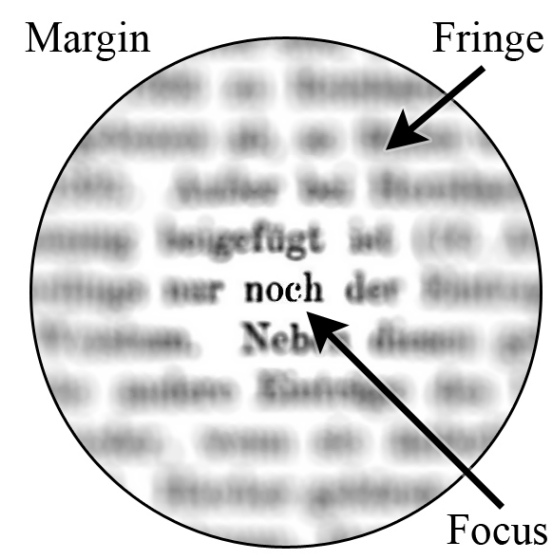

Margin

Inner thoughts Theme Physical World

Thematic Field

Bodily

Self 


\section{Phenomenology of Attention}

When it is raining in Oxford Street the architecture is no more important than the rain, in fact the weather has probably more to do with the pulsation of the Living City at that given moment. -Peter Cook, (1963) Living City: Introduction ${ }^{6}$

Psychologist William James claims, "Everyone knows what attention is. It is the taking possession by the mind, in clear and vivid form, of one out of what seem several simultaneously possible objects or trains of thought." There is a complexity and adaptability of human perception. Perception can be defined as active and information seeking within the conscious and subconscious levels, while maintaining a selective nature or interest of perception. ${ }^{8}$ James explains the self-referential foundation of consciousness:

"My experience is what I agree to attend to. Only those items, which I notice, shape my mind - without selective interest experience is an utter chaos. Interest alone give it accent and emphasis, light and shade, back ground and foregroundintelligible perspective in a word." (404)

A common assumption is that perception is determined by characteristics of external stimuli, however different people attend to different aspects. Through experience we begin to identify the variables that give us more information about our world. James describes the field of consciousness as the focus, a selected subject out of the perceptual flux; and the margin, everything that falls outside of the subject's interest. James stresses temporality over any organizational features of the field of vision. ${ }^{9}$ [See Figure 3.1].

Phenomenologist and psychologist Aron Gurwitsch's model of the field of consciousness is organized by three domains: the theme as the center, the thematic field as relevant edge and the margin whose features are irrelevant to the other two domains, while the data in the theme and the thematic field remain relevant to each other. He claims that the data concerning the physical world, inner thoughts and the body, is always present in the field. For Gurwitsch, the continuity of consciousness is overshadowed in experience by the continuity of context by the difference in degree or intensity, such as the qualities of near versus remote or intense versus faint. ${ }^{10}$

The ability to be aware of multiple domains in the field of view suggests a rich view of consciousness. This reference to a "totality of data" combines the presence of all three domains in a single conscious state. American psychologist, Michael Posner, and his experiments of the spotlight model propose the theory of spatial attention. He states that our attention moves around the visual field, often independent of our actual gaze direction. We are just as likely to notice something if it appears on the edge of our vision. ${ }^{11}$

"For a perception to be conscious does not mean you deliberately access it with the help of your attentional mechanisms. On the contrary, most things we're aware of are on the fringe of our consciousness and not in its focus. "Conscious experience generates a sense of self by placing you in the center of a behavioral space, of your consciously experienced world-model, your inner virtual reality"12

....But whatever is available for deliberately directed attention is what is consciously experienced. Nevertheless, if we carefully direct our visual attention at an object, we are constitutionally unable to apprehend the earlier processing stages. ${ }^{13}$ 

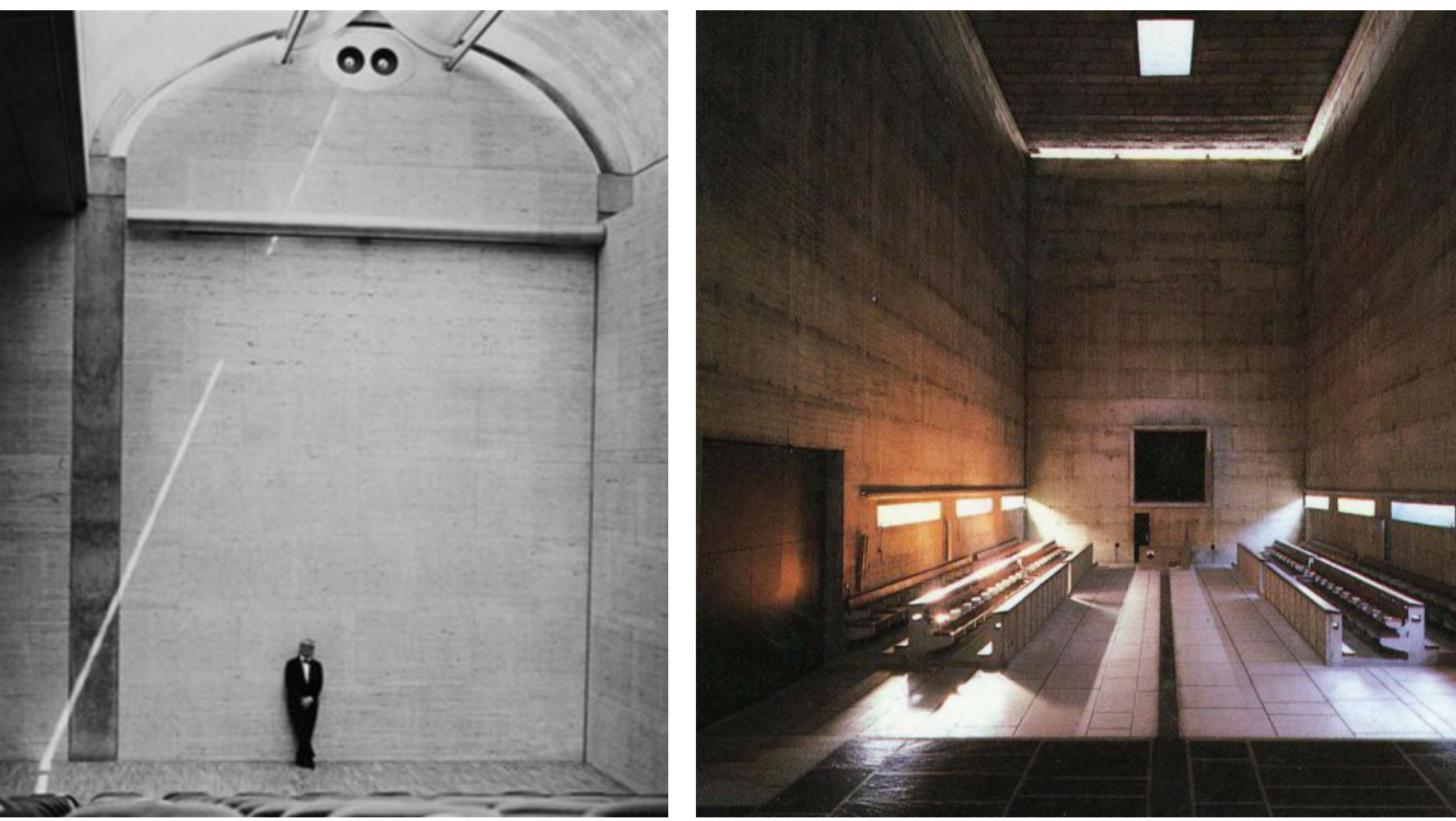

Figure 4.3 [LEFT] Kimbell Art Musuem, Louis Kahn Figure 4.4 [CENTER] Sainte Marie de la Tourette, Le Corbusier Figure 4.5 [RIGHT] Yuliio eye movement heatmap in Chapel interior

\section{Quality over quantity The quest for (sensory) experience}

The simplest nuances of sensory awareness, in the form of sight, touch, smell and taste, are indescribable because they can never be built out of mental constructs or put into words. Philosopher and author of The Ego Tunnel, Thomas Metzinger, contends that, "the contents of consciousness can be ineffable in many different ways," and as a result calls this state ineffability. ${ }^{14}$ He illustrates that we discriminate sensory values by comparison, as we cannot create concepts to describe or retranslate sensory experiences to others. Vague categorizes are not sufficient, as they are not able to capture "the concrete qualitative suchness of the experience." ${ }^{15}$ [Figure] For example, we are not able to differentiate among different hues of the same color individually. When we can see both colours at the same, we are able to differentiate between them with clear preference.

We are much better at discriminating perceptual values (i.e making the same/difference judgments) than we are at identifying and recognizing them. ${ }^{16}$

'Qualia', a term coined by Clarence Irving Lewis, is the philosophical term for simple sensory experiences that form 'recognizable inner essences'-the properties that make up the atoms of experience-deducible to instances such as the 'redness of red', or the 'sweetness of peach.' ${ }^{17}$ Metzinger further claims that we do 


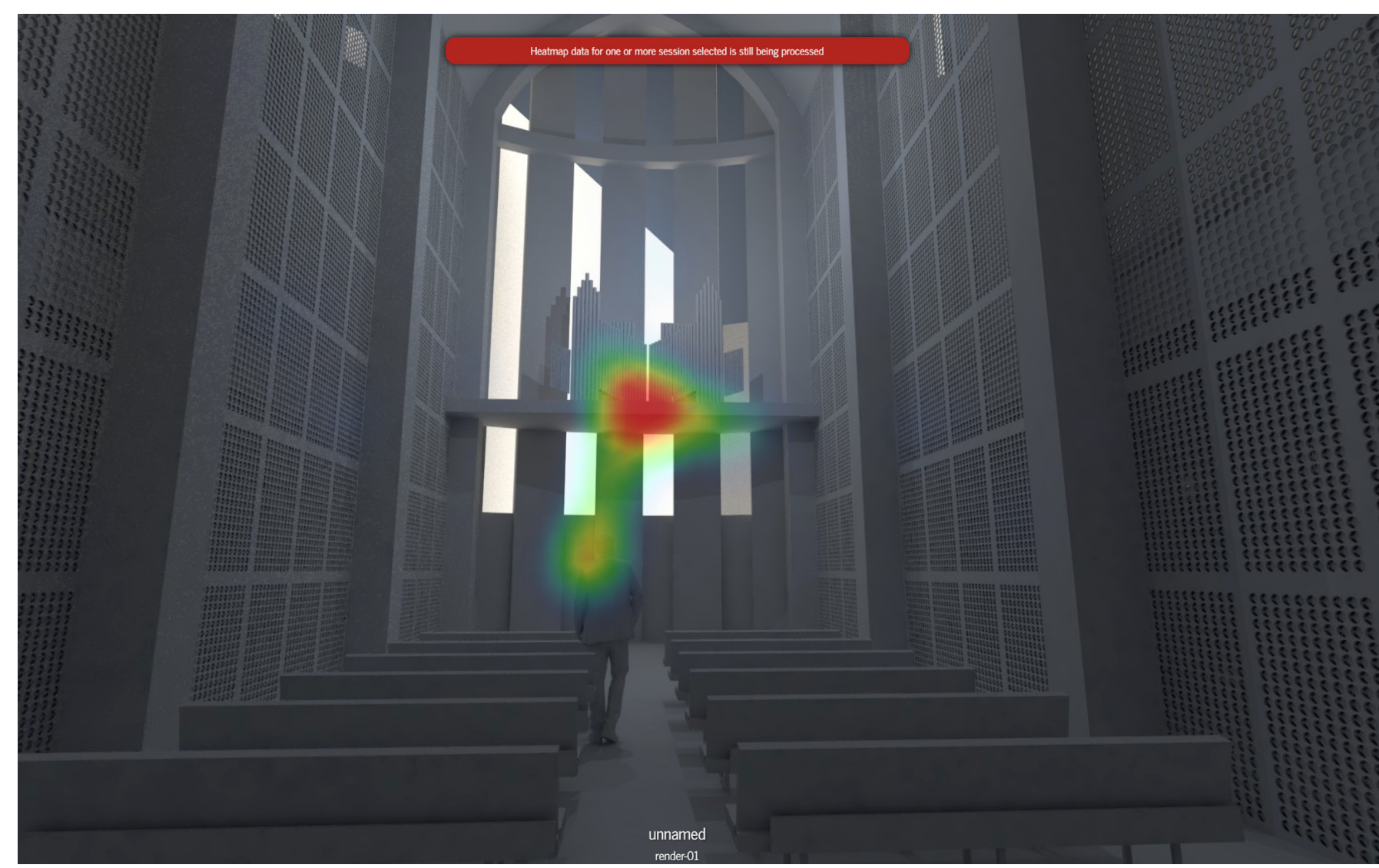

not possess 'introspective identity criteria' for the simple states of consciousness. Thus, we are capable of experiences without identifying them. His findings, "...demonstrate how subtle is the flow of conscious experience...there are innumerable things in life you can fathom only by experiencing them; there is a depth in pure perception that cannot be grasped or invaded by thought or language." ${ }^{18}$ At the same time, architecture has the capacity to recall eidetic memories of past experiences and relate to familiar places, "even unconscious archetypal places, places that we recognize like friends not seen in decades, interesting, critical of local norms." ${ }^{19}$

The 'multimodal'20 nature of perception cannot be ignored; "To get to know [these] spaces, one explores, and builds a mental map of them". ${ }^{21}$ We use a qualitative approach to evaluate our experiences and surroundings, such as good or bad, by "the information content of the incoming stimuli, and not their absolute intensity".22 In other words, in our perception of the physical world, quality dominates quantity. We evaluate the qualities of our environments based on their structure, organization and how they satisfy our needs for visual information. We seek information for a purpose (related to our expectations, senses and various qualities). The richness of environmental experience is due to the varieties of information specific to various perceptual systems. Sensory redundancy heightens our experience. For example the experience of fire is heighten by a combination of smell, sight and touch.

In VR simulations, the use of audio narratives or music can heighten spatial qualities. 


\section{Processes of perception}

\section{Perception, Cognition and Spatial Behavior}

"If we can understand the nature of what we see and the way we perceive it, then we will know more about the potential influence of manmade design on human feelings and thinking". -Walter Gropius ${ }^{23}$

Jon Lang, along with several other authors of Designing for Human Behavior (1974) theorize that there are three psychological processes that enable man to 'adjust or achieve mastery over' one's environment. They are perception, cognition, and spatial behavior. Lang notes, "the physiological abilities of an individual affect not only the way he perceives the environment but also how he thinks about it, and how he can use it." ${ }^{24}$ We unconsciously use spatial intelligence to navigate our world.

Perception of space is paramount in the parameters of human-centered design-it is a natural extension. Architects have been interested in visual perception, since current theories of "basic design" borrowed from psychology of perception looking to meet visual objectives. In practice, however, architectural theorists find greater interest in building ideological positions than engaging processes of perception, due to contradicting theories in both psychology and philosophy. Perception theories attempt to explain phenomena, without evaluating what is good or bad. In understanding the processes of perception of sensory and information based processes we can draw upon how space and place are conceived and defined, and how we can access what will be emergent when integrating virtual reality in our awareness of the totality of data.
The theories of perception can be classified into four main categories:

[1] Empiricism is defined by the work of Helmholtz,

Tichner, Carr (American functional psychologist), and the transactionalists: Adelbert Ames, Hadley Contril, Willam Ittelson

[2] Rationalism and nativism are defined by the work of Emanuel Kant, Jean Piaget, and Noam Chomsky

[3] Gestalt Theory is developed by Kurt Koffka, Wolfgang Kohler, and Max Wertheimer

[4] Information-based perception theory is developed by psychologists such as James J. Gibson and Eleanor J. Gibson. 


\section{DIRECT [ACTIVE PERCEPTION]}

(Information based)

"What is perceived?"

SPATIAL AWARENESS (perception)

Immersive scale/Sensibility to

environment (i.e context)

'Description' (in the brain)

$\downarrow$

Senses

$\downarrow$

Surrounding Enviornment
INDIRECT [PASSIVE PERCEPTION]

(Sensory)

"What is felt?"

TEMPORAL AWARENESS

Immersion/Bodily awareness

(proprioception)/Embodied action

(awareness of one's movement) ${ }^{`}$ 


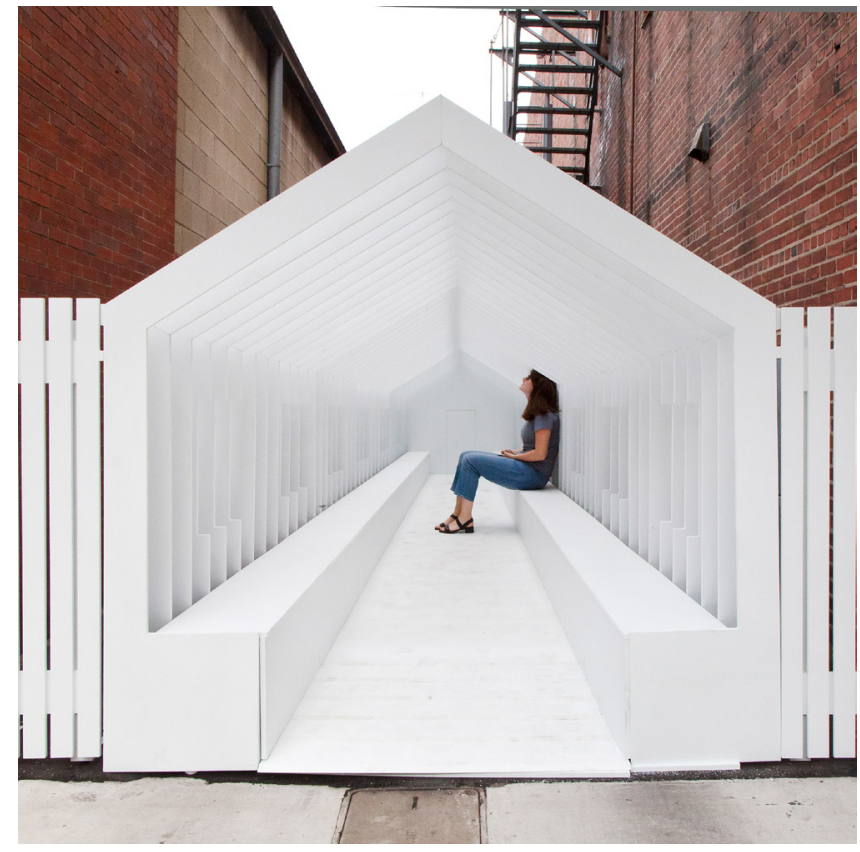

\section{Sensation-based theories}

Empiricism, Rationalism and Gestalt theories suggest that perception is based on sensation - directly affected by environmental stimuli. While each theory explains a different model of the process, each model examines the various sensations one can experience. Sensation" 25 is defined as 'the bottom-up process by which our senses, like vision, hearing and smell, receive and relay outside stimuli'. While perception is the 'top-down way our brains organize and interpret that information and put it into context'26

"Colours, sounds, temperatures, pressures, spaces, times, and so forth, are connected with one another in manifold ways; and with them are associated dispositions of mind, feelings, and volitions. Out of this fabric, that which is relatively more fixed and permanent stands prominently forth, engraves itself on the memory, and expresses itself in language. ${ }^{.27}$

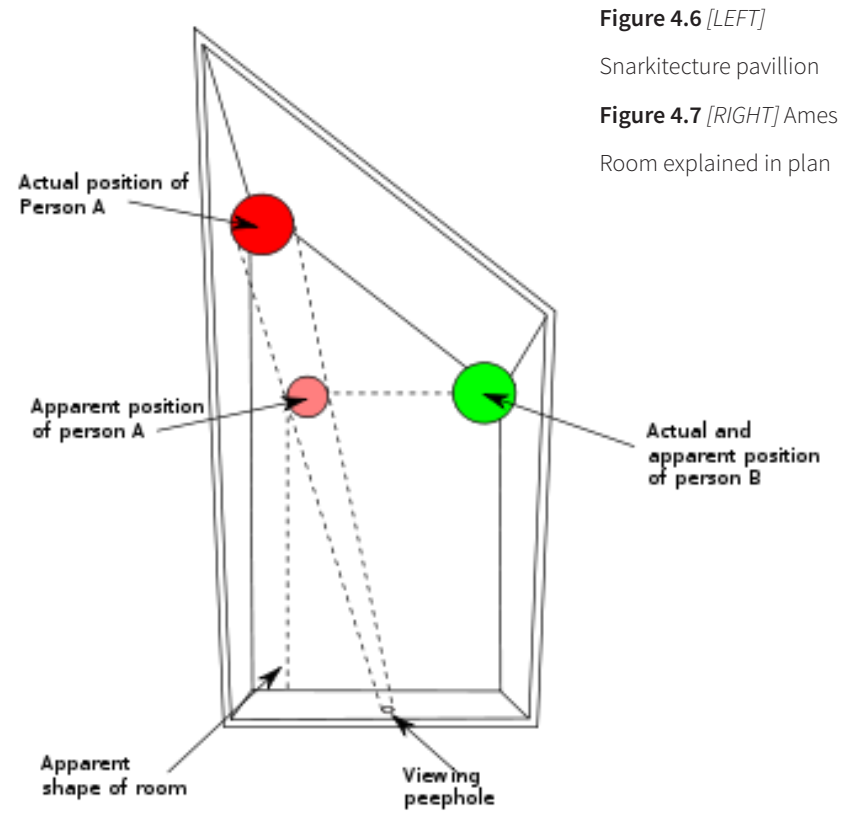

\section{Empiricism}

Empiricism uses previous sense experience. Transactionalists emphasize the privacy of an individual's perceptual world, claiming that the individual creates his/her own world based on history, motivations and values. In practice, we can see this effect in Albert Ames's experiment of the Ames room which demonstrates that 'sensation' does not come from the material world, but rather from prior experience. The experiement is also further emphasis that people attend to different things in an environment based on experience.

\section{The Rationalism and nativism Theory}

This thoery is premised on the idea that actions are based on reason and knowledge, independent from sense experience (religious belief or emotional response). The theory claims that certain elements of cognition are innate knowledge. Kant asserted that, "the mind is not merely a passive recipient in perception but an active originator in experience."28 


\section{Gestalt Theory}

We are wired to create order in the things we see. The gestalt laws explain how we perceive patterns, relying on the brain to organize data from our senses. One of the main features of the Gestalt approach is the organization of our experiences. The Gelstalt's self-organizing tendencies had psychologists concerned with direct and immediate experience.

Perceptual psychologist, Rudolph Arnheim saw that 'expressive qualities' are tied to specific configuration within experience through visual organization and architectural form. ${ }^{29}$ The fundamentals of gestalt design view the visual world as a set of points, lines, and planes of various lengths and widths, forming a variety of tensions with suggested directions and energies extending into the third dimension. Gestalt "laws of organization" (which are learned procedures of perceiving relationships) apply to contours, textures, and surfaces, rather than geometry. 9extract shapes and object recpgntion) The optical characteristics used spatial configurations include:
- $\quad$ Principle of similarity and proximity

- $\quad$ Proximity; objects that are closer together or share similarities, are 'visually' grouped together;

- Law of good continuance; the arrangement of figure-ground is most easily seen;

- Theory of closedness; areas with clearly defined contours can be seen as figures more easily

- Law of closure (Kohler); We visually group figures that are almost complete/whole, due to force's of organization

- Law of Pragnaz;

- $\quad$ Conceptual clarity: (open unenclosed space versus enclosed space): Heinrich Wolflin outlines two contrasting attitudes in architectural history: The first is that well-defined enclosing elements are defined as "classic", and the second are more loosely defined are called 'baroque., 30

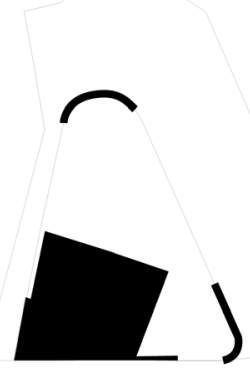

Principle of

similarity and

proximity

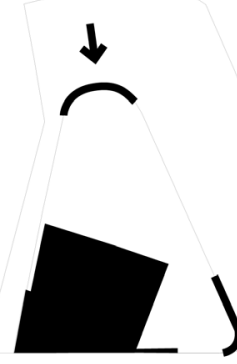

Law of closure

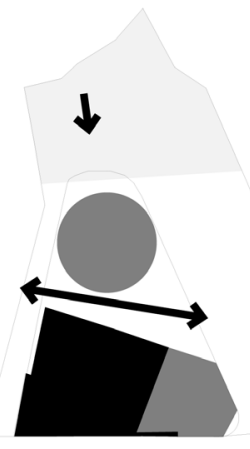

Law of good

continuance

Figure 4.8 Site Parti 


\section{Perceptual learning: Vision:}

Vision is not an independent body function. -Maurice

Merleau Ponty ${ }^{31}$

Undoubtedly, the length of a man's nose determines how he sees himself. - James Gibson ${ }^{32}$

\section{Information-based}

Cognitive theories of perception suggest a lack of stimulus, claiming that sensations alone cannot provide a description of the world, but requires 'enriching' through visual motivation. Closing the gap between sensation and perception, J.J Gibson offered an alternate theory of perception grounded in pragmatism. His cognition and ecological psychologies based on information theory is what Benedikt referred to as "the science of place". ${ }^{33}$ Information-based perception theory examines how one processes phenomenal information and the relationships that are formed based on visual perception. While the presence of sensations is not denied, the basis for visual perception in Gibson's model is registered by ambient light, rather than sensation. ${ }^{34}$ Similar to the model of perspectival geometry, Gibson's basis to perception of the visual world posits that we perceive optic arrays (a set of light rays) through ambient light structured by the difference in light intensity depending on edges, corners and other boundaries of reflecting surfaces.

Gibson defines our role in the environment as both perceiver and behaver of direct perception- both sentient and animate. Gibson removes the dependency on the eye altogether, when defining and explaining vision. There are multiple types of vision used to observe the environment, beyond pictorial depth perception; while a 'single, frozen field of view provides only impoverished information about the world." ${ }^{35}$ He claims that "the visual system did not evolve for this. ${ }^{36}$ Instead of explaining vision as a sense or tool that is dependent on the eye, Gibson defines our most commonly used perspective when there are no constraints on the visual system, as natural vision. Natural vision is connected to the head and the body able to move around; look around; and see objects from all sides.

Natural vision is not a series of compounded snapshots taken in rapid succession. The evidence suggests that visual awareness is panoramic and persists during long acts of locomotion:

"There are no sharp transitions in a medium, no boundaries between one volume and another, that is to say, no surfaces. This homogeneity is crucial. It is what permits light waves to travel outward from a source in spherical wave fronts. Indeed, it is what makes a chemical emanation form a source foreign to the medium itself, and this capable of being smelled."

(Gibson 18)

Gibson suggests that vision should extend beyond 'pictorial depth perception'. "We need to see all the way around at a given point of observation and to take different points of observation." ${ }^{37}$

Awareness on the part of the architect: It is through movement that we perceive works of architecture. Our movement is purposive and depends on our motivations. ${ }^{38}$ It is important to consider "how the formal relationships which appear in an architectural drawing will appear as we move through the environment." ${ }^{39}$ According to Gibson, the environment is what we directly perceive at any given moment. He argued that when we perceive an object, we observe its affordance, not its particular qualities the object posses. Affordances provide opportunity for action. Thus, perceptual learning is a 'process of differentiation', by attending to variables of stimulation not responded to before in the many complexities and perceptions in the environment that can be picked up by the observer..$^{40}$ 


\section{SNAPSHOT VISION}

Subject fixates on a point and stimuli is exposed around the fixation point

\section{AMBULATORY (AMBIENT) VISION}

Looking at the environment through

a knothole in a fence. Vision is

constricted as observer cannot turn

their head or get up to look around in ambulatory vision.

$\therefore$...the flux of optical stimulation contain all the information that is necessary for visual perception. Thus the primary learning experience in visual perception is not that of making associations with elemental forms but of differentiating smaller and smaller differences in qualities, features, and dimensions of variation." ${ }^{41}$

Development of an experience: Building components are not meant to be seen individually. Those that are meant to be seen as components create the environment in the user's field of perception. Intuitive design is an inherit aspect to modeling program (interface) with intuitive and clear building organization to craft an environment where people will know where to go.

\section{APERTURE VISION}

Longer period of exposure - eye

scans pattern to which it is exposed

VR aims to mimic NATURAL VISION

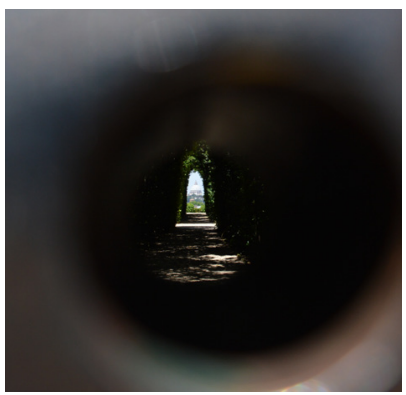

The ecological level is defined by the human respect to "behave with respect to things we can look at and feel, or smell and taste, and events we can listen to."

Figure 4.9 The Aventine Keyhole 


\section{ENDNOTES}

$\begin{array}{ll}1 & \text { William, James. } \\ 2 & \text { Lang } \\ 3 & \text { Gibson, James in “Theories of Visual } \\ \text { Perception”, Gordon, E. Ian, } 1979 \\ 4 & \text { OED } \\ 5 & \text { Lang, Jon (ed), Burnette, Charles, Moleski, }\end{array}$

Walter. "Part Two: Fundamental Processes of

Environmental Behavior," Designing for Human

Behavior: Architecture and the Behavioral Sciences.

Dowden Hutchingson \& Ross Inc. Stroudsburg,

Pennsylvania, 1974

$6 \quad$ Akten, Memo. "FIGHT! Virtual Reality

Binocular Rivalry (VRBR). - Memo Akten - Medium."

Medium, Augmenting Humanity, 26 Mar. 2017,

medium.com/@memoakten/fight-virtual-reality-

binocular-rivalry-89a0a91c2274.

7 (Cook 1963).

8 James, William. The Principles of

Psychology. Vol. 1, H. Holt, 1890. P.403-4

9 Lam, William M.C., and Christopher Hugh.

Ripman. Perception and Lighting as Formgivers for

Architecture. Van Nostrand Reinhold, 1992.

10 Arvidson, P. Sven. The Field of

Consciousness: James and Gurwitsch. Transactions

of the Charles S. Peirce Society, Vol. 28, No.

4 (Fall, 1992), pp. 833-856 Indiana University

Press. Accessed online: http://www.jstor.org/

stable/40320393

11 Vinson, W. David. Yoshimi, Jeff. (2013)
Consciousness and Cognition: Extending Gurwitsch's field theory of consciousness. Cognitive and Information Sciences, University of California, Merced, CA. Accessed online, Elsevier.

\section{2}

13 https://yulio.zendesk.com/hc/en-us/ articles/115015404287-Heatmaps-Understandyour-client-s-behaviors-and-interests

14 Metzinger 49

15 ibid.

16 Metzinger, 50 (Daniel C. Dennett,

philosopher of consciousness)

17 ibid.

18 Benedikt, Michael, On Bachelard's The

Poetics of Space Now. Centre 17: Space + Psyche, (2012) P.8

19 (Ittelson's term, Lang, 87)

20 ibid

21 Lam,

22 [Gropius, Walter. (1943) Scope of Total

Architecture, NY: Harper \& Row; See Walter Gropius, 'Design Topics'; Magazine and Art, Vol. 40 (1947).]

23 Lang, Jon. Theories of Perception and “Formal” Design. University of Pennsylvania, 84 24 Sensation, is defined as "a mental process, such as seeing, hearing or smelling, resulting from the immediate external stimulation of a sense organ often as distinguished from a conscious awareness of the sensory process." It can also be a physical feeling or perception resulting from something that happens to or comes into contact with the body (https://www.merriam-webster.com/dictionary/ sensation)

25 Youtube link: Sensation and PerceptionCrash Course Psychology \#5

26 Mach, Ernst. (1879) The Analysis of Sensations) Dover Edition (1959). Translated by CM Williams and Sydney Waterlow. Chapter One. Acccessed online: https://www.marxists.org/ reference/subject/philosophy/works/ge/mach.htm 27 (Temple, 5)

28 Levi, David. The Gestalt Psychology of Expression in Architecture. New School for Social Research, p. 111-119

$\begin{array}{ll}29 & \text { mmp } \\ 30 & \text { Gibson 208 } \\ 31 & \text { Benedikt } \\ 32 & \text { Ibid, Lang, Jon. } \\ 33 & \text { Gibson } \\ 34 & \text { ibid } \\ 35 & \text { gibson } \\ 36 & \text { (Lang, 87) } \\ 37 & \text { Gibson, J.J. The ecological. As Gibson's }\end{array}$
title expresses, the 'ecological' level is defined by the human respect to "behave with respect to things we can look at and feel, or smell and taste, and events we can listen to." (p. 22) 


\section{The architectonic, the narrative, and the poetic}

Being-in-the-world

\section{A Phenomenological Approach}

There is a phenomenological consideration to our relationships with buildings. We are fundamentally connected with the world through our senses. Architecture is perceived habitually by perception, as well as, by its use. ${ }^{3}$ This intuitive approach for users to engage with built artifacts occurs primarily through sight and touch, while our intuitive understanding is shaped by our cultural, educational and ethnic experiences. The phenomenological qualities of a built environment mediate the interface between the human body and our world/reality. Dalibour Vesely explains phenomenology as,
"Phenomenologically, the greatest loss is that the 'sense of place' cannot be experienced. Thus, any technic (in the above case, the telescope) in amplifying an isolated sense, or senses brings with it the concomitant loss of reduction in total experience' - Peter McLearly ${ }^{1}$

"Anyone who has first seen a place in a picture and then visited it knows how different reality is. You sense the atmosphere all around you and are no longer dependent on the angle from which the picture was made."

-Steen Eiler Rasmussen²

\section{...an attempt to understand from the inside-and not to dismiss or criticize from the outside-the whole spectrum of the current experience which we generally call 'reality.'} Dalibour Vesely addresses the term 'reality' in much broader context than the practice, extending to culture, frame of reference and questions in the area of culture. For Dalibor Vesely the primary purpose of representation "is the mediating role, which can be described as participatory because it enhances our ability to participate in phenomenal reality." 
Our experience of the given reality is never direct, but only mediate, and that the most important role of representation can bring us closer to the depth and the plenitude of phenomenal reality, which would otherwise remain inaccessible. ${ }^{6}$

The emergence and development of phenomenology drew influence from 'a crisis in perspective' in reaction 'against the insufficiencies of psychology.'? In the late 19th and early 20th centuries, the proliferation of architectural styles and artificial settings of international expositions called for a 'common communicative domain' in search for a larger organizing system, philosophers took it upon themselves to 'rethink the nature and meaning of measure through a phenomenological approach to the world". ${ }^{8}$ Phenomenology developed in tandem with modern culture, conceptual direction, culture, symbolism, and meaning; where the 'second level of phenomenology is everyday culture.' The construct studies the structure of various experiences of perception, thought, memory, imagination, emotion, desire, and volition to bodily awareness, embodied action, and social activity.

Phenomenology is rooted in the imaginative praxis leading in two directions: ${ }^{10}$

[1] Outward, fantasy trajectory

(Husserl Elevated imagination over perception)

[2] "Material" trajectory

(existential and hermeneutic phenomenologists MerleauPonty and Heiddger)

Husserl founded phenomenology, struck by the incomparable measure between essence and fact, in that, by beginning research with facts, one will never attain to its essence. ${ }^{11}$ The principle of phenomenology is founded upon eidetic intuition. Sartre claims

"...room must be made for the experiences of essences and values.
We must even recognize that essences alone enable us to classify and examine facts." ${ }^{11}$

Husserl questioned what happens when something appears to someone. In Husserl's view, to discover the phenomenon of the house, we must ask what makes a house a home. By exploring the mechanism that helps understand the existence of phenomena, the human consciousness is no longer magnetized by mere facts. Conversely, Heidegger believes that the experience of the world (praxis) precedes, rather than follows, perception. ${ }^{12}$

For Merleau-Ponty, the body is the primary site of knowing the world. "indirect ontology" the body and what is perceived cannot be separated. Merleau-Ponty believes that vision is 'an act of two facets': "the inner horizon of an object cannot become an object without the surrounding objects' becoming a horizon."13 This view of perception can be best understood in the example of film. In film, when a camera zooms into a close-up view of an object, we can remember what were shown, even if "we do not actually identify it". ${ }^{14}$ This occurs because the screen has no horizon. The horizon guarantees the identity of the object throughout perception.

The movie is for the absent-minded examiner, not for the participator. As eyes 'grasp' the scene, thought is occupied with perceiving moving images. "The spectators process of association in view of these images is indeed interrupted by their constant, sudden change." Whereas, art demands concentration, "A man who concentrates before a work of art is absorbed by it". ${ }^{15}$

VR for architects is like film for directors: self-referential system of perception that allows one to think and feel, activating a mode of participation. It allows the perciever to experience the product with the ability to implement selective, as well as periphery attention. Thoughts are not replaced by moving images such as those provided by film and there is no need to relate 


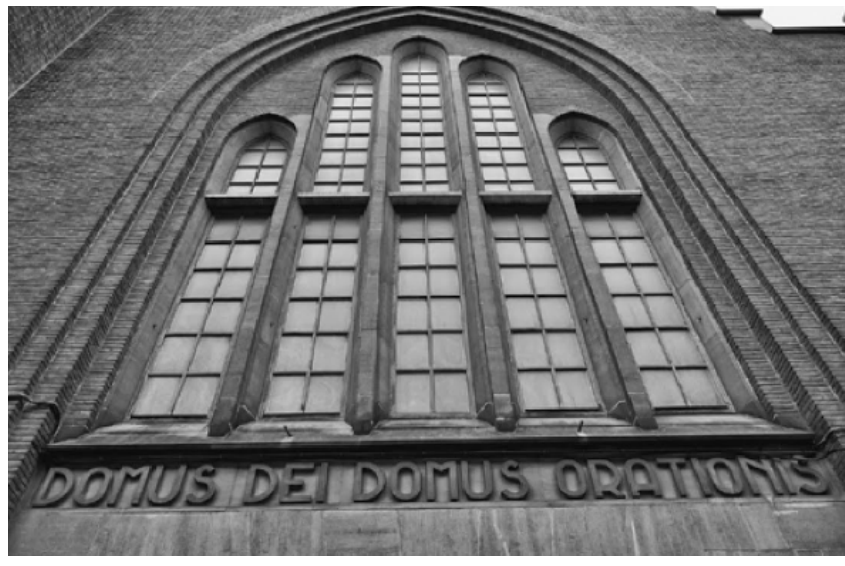

Figure 5.1 Domus Dei, Holy Heart Church (Brussels, Belgium)

separate images to combine an impression. Changing the role of the viewer to the visitor-and then to the navigator, allows one to not only to see the [virtual] environment, but also to feel it. The interaction with space at the level of the city, the building, and the body (human scale) mediates the digital and physical world, rendering digital space as digital place.

\section{Being-in-space -Places of worship}

In the Middle Ages the greatest building in the city was the church or cathedral which was seen as the Domus Dei, the House of God. In the 1950 the 'House of God' was used as identification of a public space where everyone including the rich, the poor, the king, the serf, the bishop and the laymen were welcome: "It was a beginning of a consciousness that there must be at least one communal space that everybody can call home." ${ }^{16}$

Today, the need for 'spiritual' space remains relevant, as we seek spaces to self-reflect, meditate or be mindful. Spirituality is, 'the quality of being concerned with the human spirit or soul as opposed to material or physical things"17 The concept of spirituality has many meanings and is used across multiple contexts, such as religion, architecture, music, painting, literature, philosophy, and alchemy (spiritualism, astrology, esoteric knowledge). Spirituality can be defined as your awareness that your life is animated by 'unseen realities', such as love, joy, and gratitude. Spirituality is about engagement of life. Author of $A$ Design Manual for Sacred Buildings, Rudolf Stegers claims,

Even today, spaces of worship with architectural aspirations must fulfill not only functional but also atmospheric expectations. They should help the 'user' to reach what the protestant theologian Paul Tillich described as 'the state of being grasped by ultimate concern.18

How we chose to represent spirituality and how we choose to engage in spirituality is reflected in one's perception and beliefs. Russian movie director Andrei Tarkovsky known for creating spiritual cinema, found a great interest in matter and detail. To portray spirituality, his heroes don't pray looking up; instead, they immerse their faces in mud, praying to the ground. ${ }^{19}$

There are, indeed, things that cannot be put into words. They make themselves manifest. They are what is mystical. ${ }^{20}$ Sensory experiences contain qualities that "bloom up from the universe to greet the close attention we give to any object, mechanism, or activity". ${ }^{21}$ Presence and mindfulness in grounded in feeling the weight of the present moment. There is space for us to lean into. The interest in the places of worship and spiritual space is linked to the sensory qualities expected to manifest in the physical and material in its presence. Among the occasion search for meaning and thirst for wanderlust, we are continuously worshipping technology, ${ }^{22}$ as "undivided attention is a form of prayer" 23

\footnotetext{
In cinema we do not perceive reality-but inteasd, we perceive representations, constructions, and fabricatiosn (copies and reproductions) Cinema synthesizes reality. It demonstrates a superior perception. Cinema consciousness is 'the camera, now human, now inhuman, or super-human' ${ }^{24}$
} 


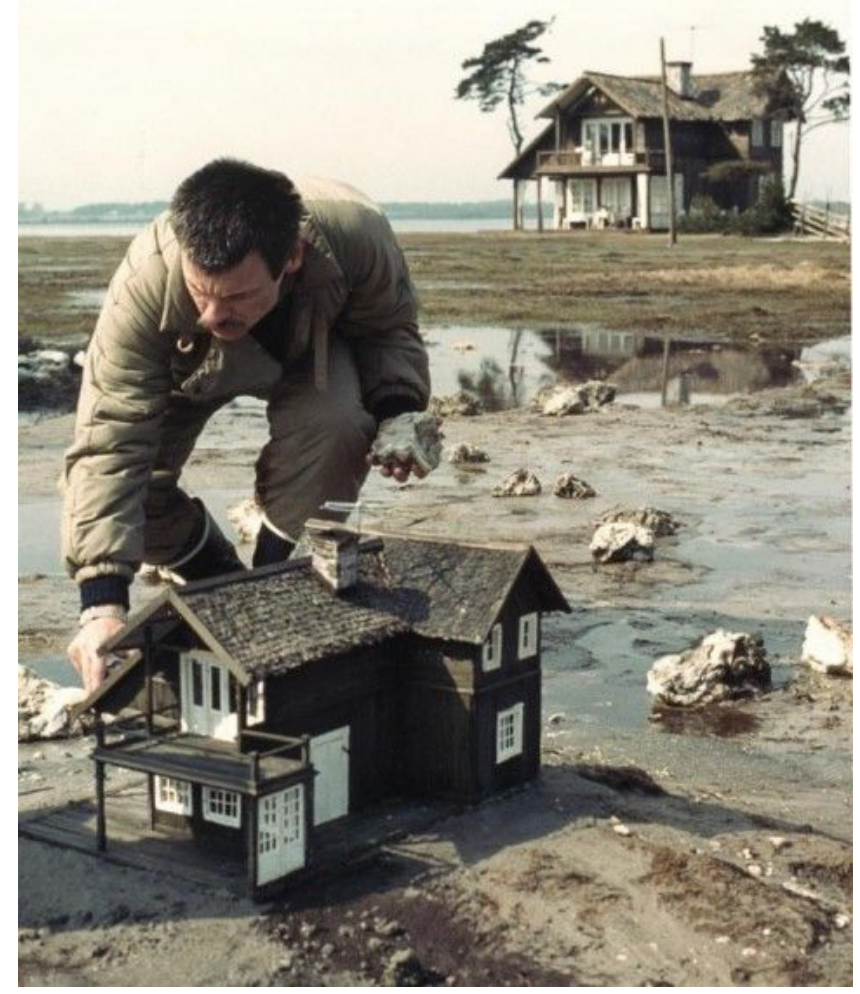

Figure 5.2 The Spiritual Cinema of Andrei Tarkovsky. Tarkovsky on the set of Nostalgia with model.

On the subject of the world of appearances and assumptions of reality behind those appearances, Mark Foster Gage architects work within an aesthetic philosophy, questioning how one relates to appearances and the realities behind them, by proposing: [Architecture that] make[s] you question your assumptions of how you occupy the world; [architecture that] makes you want to learn more; [architecture that] wants you to de-stabilize your assumptions of reality... to take apart their assumptions of what architecture is. ${ }^{25}$

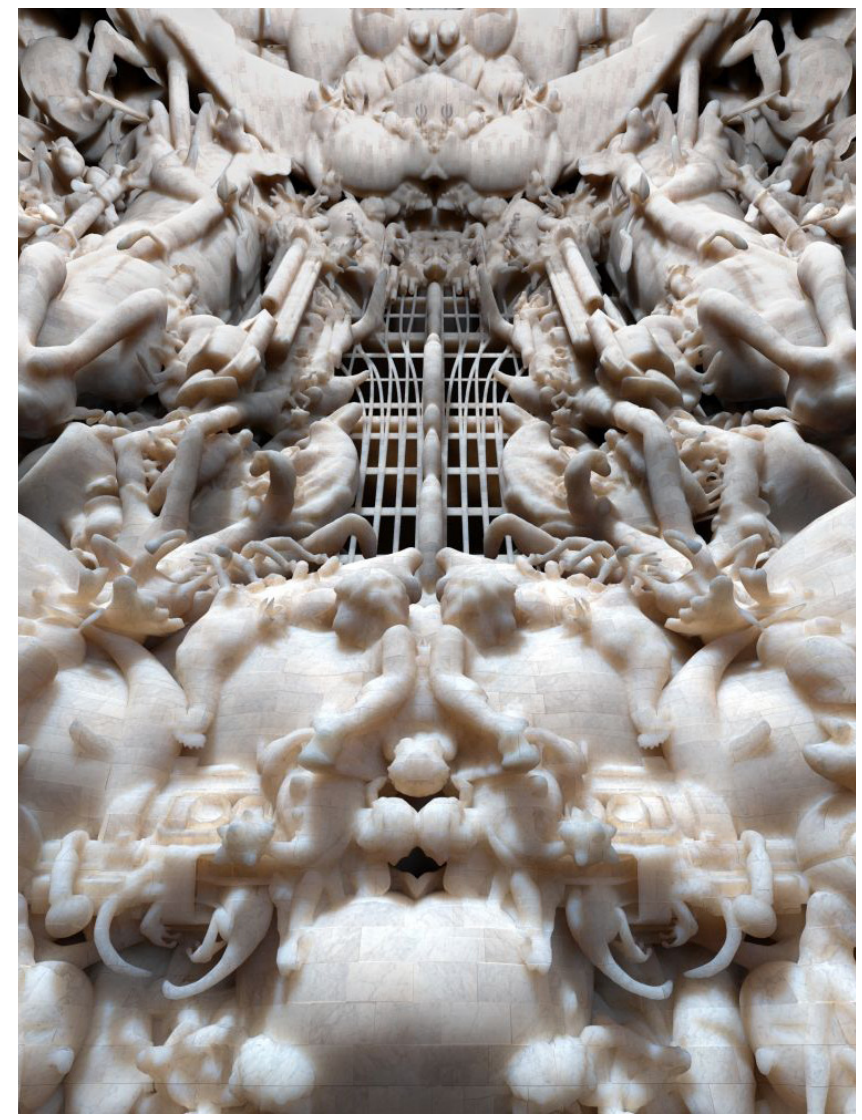

Figure 5.3 Mark Foster Gage architects, competition entry, 2016

At a talk in TEDxMidAtlantic, through his work, Gage demonstrate that instead of following the historical "form follows function", form should follow "vastness" in an effort to expose people to a much larger reality. He claims, "Architecture should be an ethical reminder of the things which are greater than us; and in doing so, make our differences seem less apparent."26 A major theme of the work is working at various scales. Gage architects play with details providing sensory stimulus that we unconsciously all crave. 


\section{Design project}

The chapel is a place of prayer and worship. Derived from the Latin word, cappella (chaplain), a chapel is defined as "a small building for Christian worship, typically one attached to an institution or private house."27 It can be part of a larger nonreligious institution, or an extension of a primary religious institution, or an extension of a larger complex such as a hospital, prison, educational facility, church, synagogue or mosque. THe design project engages a completely free-standing structure. The chapel used as a vechile in the design research aims to address the sensory qualities that are engaged in buildings programmed for awareness of presence and mindfullness. 


\section{Architectonic qualities}

'Artshould not be explained, it should be experienced"

-Steen Eiler Rasmussen ${ }^{28}$

Spaces that create an emotional, sensory impact have a continuum of spatial experience between the intimate and the immense with celebrated spots at varying scales. Powerful spaces affect us through three levels of expression: the architectonic, the poetic and the narrative. ${ }^{30}$ "Narration can simulate experience whereas text can merely represent it." ${ }^{11}$ The construction of the virtual world is that added layer of experience within vr, which acts beyond representation and communication.

"An understanding of good design comes not only from one's professional experience of architecture as an abstract, individual pursuit, but also from one's shared, everyday experience of architecture in real time-its particular use of light, color, shape, scale, texture, rhythm and sound." (Rasmussen 9)

In 1958, in a lecture at RIBA, Steen Eiler Rasmussen shared his research and thinking on the fundamental architectonic qualities that help to kinesthetically build our architectural intelligence:

[1] Our appreciation of whether a space has been carved, moulded or surfaced.

[2] Scenographic procession through colour planes,

[3] Scale and proportion,

[4] Rhythm,

[5] Texture,

[6] Light and shade,

[7] Colour,

[8] Sound

All of these qualities help us to kinesthetically build our architectural intelligence using our sense and perception. ${ }^{32}$ 


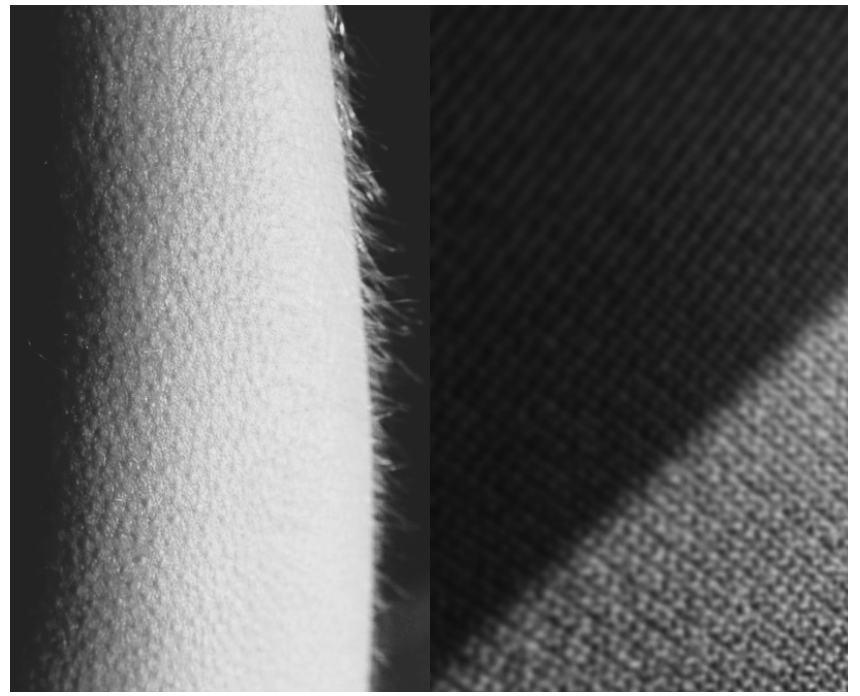

\section{Atmospheric expectations 'The Poetic'}

In an analysis of Bachelard's writing, Benedikt traces the Bachelard's homology between our perception of space, our way of being-inspace, and poetic thought:

"A poem, for Bachelard, is not a record of "impressions." Impressions can be numbered and catalogued. So too can images be, like slides. But a poem, like a building, is meant to be lived in time, in Bergsonian durée. The durée that a poem makes is much longer than the time it takes to read; the space a poem makes is larger than the space it takes to print it. (What could be more "economical"?) The poem demonstrates how inner vastness can issue from the most intimate and solitary of activities: reading." (Benedikt 2)

Benedikt claims, "humans have a penchant for experiencing immensity. These experiences are humbling, we like to say they make us feel small and finite, which is a Good Thing in the religious view." 33 Bachelard introduces the concept of "intimate immensity", ${ }^{34}$ referencing the idea that we not need a large number of trees to

\section{Figure Skin}

feel immensity. An important component of "intimate immensity" is the immensity's immediacy. The juxtaposition and continuity of narrative in perception engage our sensory experience in different ways. The speed with which we 'read' the images is influential. 1/10 the speed creates a dream-like quality. Movement or blur causes an illusion of movement.

We come away from experiences of immensity, ourselves enlarged, as though we knew that the world is neither small nor large without us to say so, that immensity is a sensation generated within ourselves. Motor activity keeps us knowing our size. Stop to gaze, meditate, or daydream, and the mind naturally expands: "(w)e return to the natural activity of... magnifying being." (Bachelard 184).

Visual experiences are common triggers for the sensations that Bachelard proposes. He states,

Self-expansion is our purpose, not self-diminution, and a certain pride in seeing, seeing at all (Bachelard 190). 


\section{Implementation in VR}

The design research will investigate the parameters introduced in the previous chapter to enhance design qualities relative to formal perception. Through the design of a contemporary chapel in Rome, Italy, the sensory qualities evoked in spiritual spaces will be simulated within the digital/virtual, adding a rudimentary layer of phenomenological experience. Informed by formal perception and Rasmuseen's fundamental architectonic qualities, the project will examine the five main principles for spiritual spaces that fuel sensory/emotional experiences visually through the architectonic, the poetic and the narrative.
1. Scale:

"Images, objects, and actions have different meanings at different scales, especially relative to the body." A dozen axioms of interaction design practices, Digital Ground

2. Boundary

Looking at spatial intelligence with virtual space as can transalte to physical space. Such as restricted space; enclosure/openness, proximity, privacy, spatial qualities (gestalt)

\section{Lighting conditions}

The presence and absence of light and shadow and all the scales in between. There is no form without light, as it reveal it. Examining the material filtering of light (permeability)

4. Materiality

Examining "perceived space" in object and background, how surfaces of gestalt reflect light and how different materials and colours choices produce different spatial qualitites.

\section{Sound}

Audio can be added to virtual reality experiences to heighten immersion. However, sound can also be a visual quality. ${ }^{34}$

French-Canadian architect, Roger D’Astou, know for designing the Notre dame des Champs, Catholic Church of Montreal, explained the feeling of a church architecture:

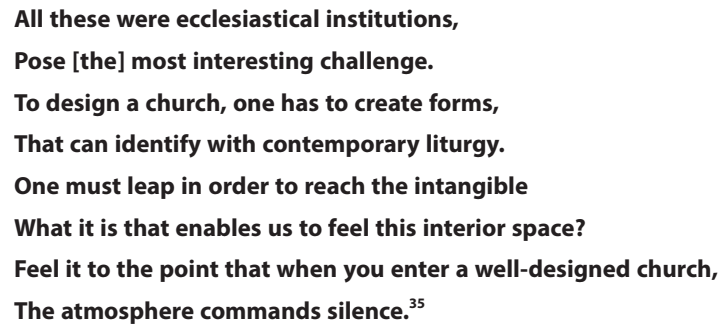




\section{ENDNOTES}

1 McLeary, Peter. "Some Characteristics of a New Concept of Technology" Rethinking Technology: a Reader in Architectural Theory. Routledge, 2007. p330

2 Rasmussen, Steen Eiler. Experiencing Architecture. MIT Press, 1959.p 40 3 Benjamin, Walter. The Work of Art in the Age of Mechanical Reproduction. Prism Key Press, 2010. p38

4 Vesely, Dalibor. Architecture in the Age of Divided Representation: the Question of Creativity in the Shadow of Production. The MIT Press, 2006. p19.

\section{$5 \quad$ Vesely 18}

6 Sartre, Jean-Paul, and Philip Mairet. Sketch for a Theory of the Emotions. Routledge, 2014, p21. 7 Temple, Nicholas. Disclosing Horizons: Architecture, Perspective and Redemptive Space. Routledge, 2007, p15.

8 Ibid.

$9 \quad$ "Phenomenology." Stanford Encyclopedia of Philosophy, Stanford University, plato.stanford. edu/.

10 Ihde, Don. Phenomenology and Arch, Albany: SUNY Press, 1986.
11 12

13 14

16 Bleeckere, Sylvain de, and Sebastiaan

Gerards. Narrative Architecture: a Designers Story. Routledge, 2017.

\section{Addington, Softspace 40}

18 Stegers, Rudolf, A Design Manual: Sacred Buildings. Birkhauser. Basel, Switzerland, 2008.

19 "Slavoj Zizek - The Reality of the Virtual

-Part 1." YouTube, 2010.

20 Wittgenstein ends his work on the realtionship of languge to the world with this inspired statment written in the midst of WWI, rooted in spiritual and moral reasoning. See Wittgenstein, Ludwig. Tractatus LogicoPhilosophicus. Routledge, 1988.

\section{Benedikt 2}

22 Technological Touch

23 Jason Silva quotes Simone Weil in interview; see Weil, Simone, and Siân Miles.

"Attention and Will” An Anthology. Penguin Books, 2005.
24 Deleuze in Rushton, Richard. The reality of film: Theories of filmic reality, 2011.

25 Gage, Mark Foster, "Architecture that challenges your concept of reality," Tedtalk, 2016

26 ibid

27 "Chapel." Dictionary.com.

28 Rasmuseen 9

29 Bachelard qoutes Valles, see Valles, Julès, L'enfant, p238.

30 Schaik 85, See Aaron Betsky, Violated Perfection, Rizzoli NY, 1990).

31 Brott, Simone, "Architecture as media and the War of Presence" Architectural Theory Review, 2009.

32 Schaik 85

33 Benedikt 2

34 Bachelard, Gaston, and M. Jolas. The Poetics of Space. Penguin Books, 2014, p183-210

35 Schaik warns that we carry manufactured sound with us everywhere, such that sound has lost its physical dimension-its 'spatiality', while space has lost its "aurality", Schaik 10.

36 Roger D’Astous. Dir. Etienne Desrosiers. Perf. Roger D’Astous. Documentary, 2016. 


\section{Functions across scale}

\section{The impacts of materiality, permeability, and boundary}

\author{
Projection is identified with the space of representation; the \\ site of ontological continuity between universal ideas and \\ specific thing"-Idea and experience, linking time and place. \\ Alberto P-Gomez $6^{1}$
}

Prior to the Renaissance period, architectural drawings were rare. In the Middle Ages, buildings were not conceived as a whole and scale was not implemented. The Gothic architecture period exercised a constructive practice applying rules of geometry and tradition on site. 'The master mason', also known as the 'Architect of the Universe' responsible for the 'model of the city of God on earth', was responsible for completing the project. The Romanesque, the Gothic and the Renaissance architecture in Europe can be dissected and classified by region categorizing the metal spaces of each region that drew inspiration from each other, for example this can be seen in the uniformity of 13th century churches in Romania. ${ }^{2}$ However, there a various expression of Gothic cathedrals in Europe of different generations and methods of traveling masons.

$$
\text { Contemporary practice has been redefined with }
$$

computer-aided design (CAD) and Building Information Modeling. The contemporary process of architectural design is composed of a conventional set of projections-construction documents-used for accurate transcription; designing and representing at the level of the screen interface from site context to detailing of various parts to makeup a whole building project. McLuhan warns that any extension of man is met by an extension of scale:

This is merely to say that personal and social consequences of any medium- that is of any extension of ourselves- result from the new scale that is introduced into our affairs by each extension of ourselves, or by any new technology. (McLuhan7) 


\section{Level of sensitivity and sensibility}

Let's begin with a definition of scale.

Scale relates to measurement. Measurement

instruments that are used by designers to make proportion drawings that are different in size, but not in form from what they represent. Scale can also indicate a change from one physical scale to another. Most notions of scale are based on the size of something as it relates to something else-which may be physical or an idea, such as an expectation of an appropriate size of an object. Orr defines architectural scale as:

...the aspect in architecture that makes buildings intelligible to us [by giving] us a sense of how to relate to the building, and it does so in a way that either attracts us and reinforces our values or repels us and contradicts our values. (Orr, 9) Scale has the capacity to extend our spatial thinking and show us alternatives to our current values by exercising our personal judgment about our values with the information available to us, on what they will be like and how they will be expressed. ${ }^{3}$

"Good scale in architecture, then, may be said to describe a relationship of visual and textural elements to the whole, to each other, and to the human participant/observer's sense of visual satisfaction the wholeness and fitness of the constructed design." (Orr, 11)

The design of architectural relationships has been primarily intuitive in past history. For Orr, the underlying definition of scale in operable form is the test of "fitness", which is not about how things relate, but how they "fit", as individual parts and a completed whole. By recognizing scale in design, designers can become more sensitive to the possibilities scale offers and proficient in their expression. Sensibility is the ability to receive sensations and awareness of and responsiveness toward something, such as an emotion. In 1:1 scale immersion, we can engage the understanding of variables and principles and their real-life (scaled) application, such as hierarchy or sequence, rhythm/repetition, precedence/ emphasis, tension, and time. Recognizing a building on an intuitive or subconscious level reinforces our feelings of security and well-being. We are comforted by a building's 'fitness' and knowing where we fit in that scale makes feel at ease, whereas not knowing can create a condition of the intimately immense. Orr believes that "fitness" of scale should be one of the primary goals of architectural design, even though it is judged on a personal and subjective level. However, designers are after good scale:

"Good scale is achieved when personal insight and the wisdom of the ages are integrated into the design process to produce wholeness, beauty, pleasing visual experience, and satisfaction in using the creation so designed" (Orr, 11) Building design 'that handles scale well' will be perceived as a gestalt: 'a structure whose totality is greater than the sum of its parts-because the functions of scale operate in simultaneously supportive ways'. On the other hand, bad scale equates to 'unfitness' in the absence of gestalt qualities. There are three sources of scale in architecture 4 : [1] in the inherent scale-giving qualities of the materials used, [2] in the natural world, and [3] in the human body

Our perception constantly relates and compares external forms to the dimensions of the human body, founded on our awareness of our own physical size. A well-known example is Le Corbusier systems of measurement based on the human body. Along with the measure of the human body, elements have scalegiving qualities such as a doorway or doorknob [See Figure 5.1]. When we perceive/understand the scale of an object, by extension we understand the scale of the room. Sensitivity to the subtleties of scale allows us to experience a tactile relationship with building surfaces without contact.

Our level of engagement differs at different distances, 
especially when perceiving scalar elements. The closer we are to a building, the more important our personal and intimate levels of human and physical relationships to the building become, such as the relationship of the size of a building component to our anatomy. When we are divorced from the building, our concerns related to non-human factors are increased. It is easier to distinguish and recognize patterns of a system or design in orthographic drawings or from a bird's-eye view of a terrain.

\section{Expectation: Image, text, and object among uncertainty}

\section{Expectation pervades our perception. A key source for}

understanding scale presents itself in our expectations. Gombrich argues that "pure", or neutral observation does not exist in science or art. ${ }^{6}$ Instead, he claims that all observation is predicated by hypotheses, and in their own fashion, molded in expectations.

"Only through testing hypotheses do scientists and artists amend their already perceived picture of reality." (Gombrich6) Our expectations are grounded in our previous experiences that we can either relate to or be challenged by, such as when we encounter distorted or unconventional sized elements, prompting our critical judgment. Experiences and exposure to various experience help to evolve and reshape our thinking.

Gombrich asserts, "One cannot insist enough that the art of perspective aims at a correct equation: it wants the image to appear like the object and the object like the image."' A direct example is Brunelleschi's mirror experiment. He further contends that perspective depends on certain expectations of the beholder, which includes 'the size-distance ambiguity', where a viewer estimates the distance of an object by how large or small it appears. Two dimensioal renderings overlay subjectivity and take advantage of this assumption.

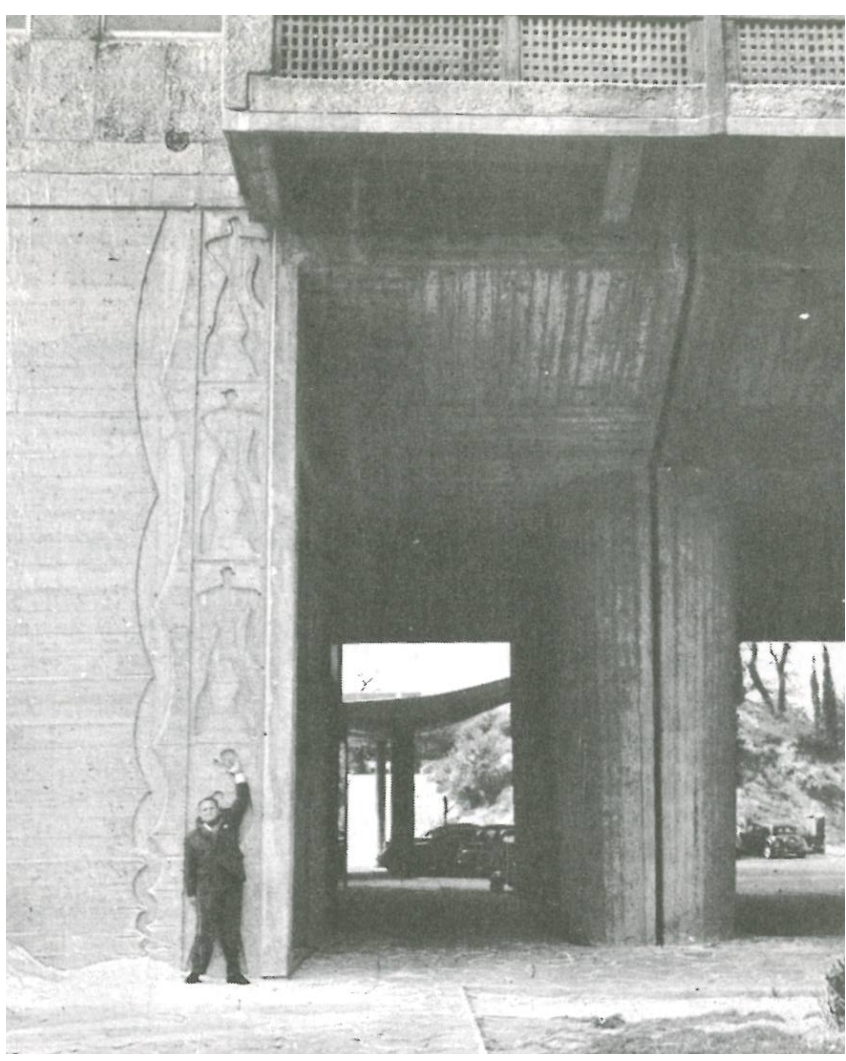

Figure 6.1 Le Corbusier Marseille block is ‘four man' tall.

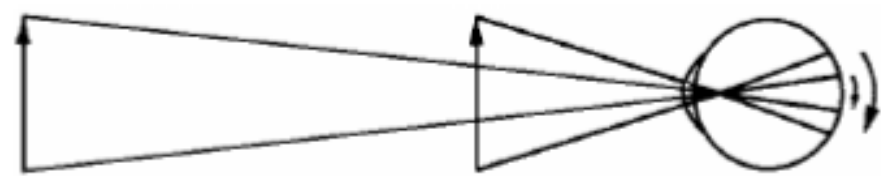

Figure 6.2 The reduction of image size with distance. 


\section{Functions and Qualities of Scale}

The function of scale is to "communicate to the user/observer specific vital facts and notions about the nature of the building that are not fully provided by other aspects of physical form (size and shape)." ${ }^{8}$ When we regard scale as a systematic expression of measurement, size can be characterized as a function or part of scale. However, both size and scale individually offer more than the features of the other, so size and scale are interdependent.

The most general function of scale is to communicate the nature of a building or a space by evidencing the designer's consideration and discretionary use of the architectural variables at hand: materials, technology, form, size, colour, texture - in short, the elements and principles of design. (Orr 26)

Therefore, FPRs and VREs become evidence of the designer's considerations and evaluation of variables at play. Strong and visually stimulating FPRs challenge awareness and attention, whereas poor and discontinuos design is uneasy to perceive when attention is scattered. With simulating FPRs the unexpected is revealed and can be evaluated for poetential redesign.

\section{ENDNOTES}

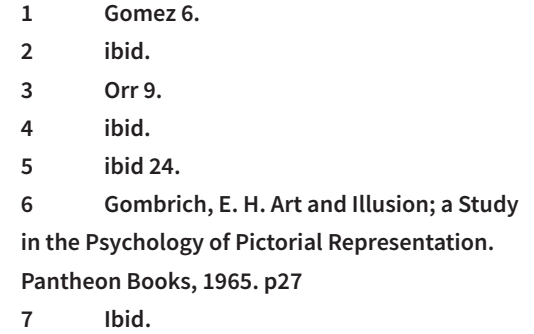
Ibid. 


\section{PART III: DESIGN PROJECT}

06 Approach: The Four Scales

07 Narrative Scale: Creation of experience

08 Immersive Scale: Environment to be perceived

09 Relational Scale: Activity of perception in visual Awareness

10 Building Scale: Information for perception 


\section{Approach:}

\section{The Four Scales}

\section{The impacts of materiality, permeability, and boundary}

From the three levels of architectural expression of the architectonic, the poetic, and the narrative, when experiencing design in VR, it is important to access the function of scales of: how the design fits into the environment; with itself (at the scale of individual component/ parts and the complete/whole building); with those who view it (frame of reference as there is no one guiding your view, and the navigator selects what they give attention to); and as a narrative (i.e. teleportation, threshold, visual navigation between spaces). A phenomenological, qualitative approach includes an intuitive combination of four scales that engage design:

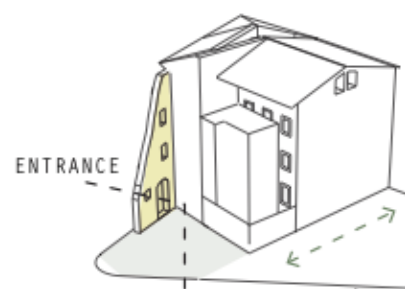

At the level of the city At the level of the body At the level of the building At the level of the sublime

Figure 7.1 Existing context on site 


\section{At the level of the city \\ Looking at the generative power of city and site}

[1] Narrative Scale indulges experienced design in sequence

- Expresses qualities of experience; hierarchy of spaces

or parts or of the sequence of movement within or about a building through teleportation and various fixed points of reference

- Provides consciousness of the flow of space guided by thresholds, doorways and openings. Offers the ability to build a sequential narrative and access their connection and navigation. Develop a sense of narrative, procession/ sequence.

\section{At the level of the body}

We always searching for the horizon. Connecting movement of the head-on-the-body with what is percived in VR from a central POV.

[2] Immersive Scale informs the 'environment to be perceived'

- Expresses qualities that are informative of a building's context and orientation: characterizes a sense of place, orientation, and how the building fits within a contextual environment
FIVE KEY PRINCIPLES FOR DESIGNING SPIRITUAL SPACES EXPRESSED IN EACH SCALE:

\section{1) SCALE IS ADDRESSED AT ALL FOUR DESIGN LEVELS:}

Narrative, Immersive, Building, and Relational

\section{2) SOUND}

Ambient sounds heightens visual immersion. Engaging multiple senses enhances our perception of the experience. 


\section{At the level of the building}

Relating a building's parts to its whole and to each other

[3] Building Scale (Scale of Fitness) brings forth 'information for perception'

- A building's openness or enclosure (evident in light penetration or permeability)

- Evauluating orientation (proximity, privacy, boundary) and spatial intelligence

\section{At the level of the sublime}

Exploring a sense of wonder, a sense of awe, the smallness of what we are, or even Bachelard's 'intimite immensity'

[4] Relational (Sensory) scale reveals the 'activity of perception' when we relate our understanding of scale to what we percieve while immersed

- Expresses qualities that are informative of a building's atmospheric qualities or overall character

- A building's monumentality or diminutiveness, such as the smallness of what we are, a sense of 'awe' or wonder (i.e Bachelard's intimate immensity)

- Allows us to know where we fit at that scale and how the building fits together with those who navigate it in VR.

- Experiential sense of atmosphere created by the qualities of materiality, light, and colour.

\section{5)LIGHTING CONDITIONS}

Sublime qualities of space use light as a material.

\section{1) SOUND}

Sublime qualities of space can support the absence of sound. Sound can also reveal the voluminousness of space. In the context of VR it is accompanied by audio files to enhance immersion for the perciever. 


\section{Design Intentions}

The design intentions are:

[1] To revitalize the public square in order to generate greater interaction between inhabitants and visitors

[2] To repurpose the existing wall ruin, and knit it and new architecture into the existing context

[3] To consider the 360-degree of the visual experience of place beyond what fits into a render frame

[4] To create a narrative experience that questions where we 'fit' at various scales, in attempt to challenge human scale and perception 


\section{Design Project}

The modern chapel is composed of three parts.

[1] The existing wall ruin becomes the new entrance to the café and patio above. The entrance gives access to an introspective multi-floor viewing terrace of the new garden and creates views across the river. The entrance visually navigates the visitor underground to the subterranean exhibition gallery.

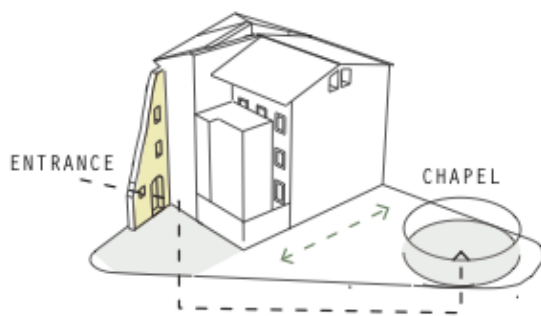

[2] The subterranean exhibition gallery links the entrance café with the chapel. The gallery serves as an underground connection and is flexible for chapel events, exhibitions and gatherings.

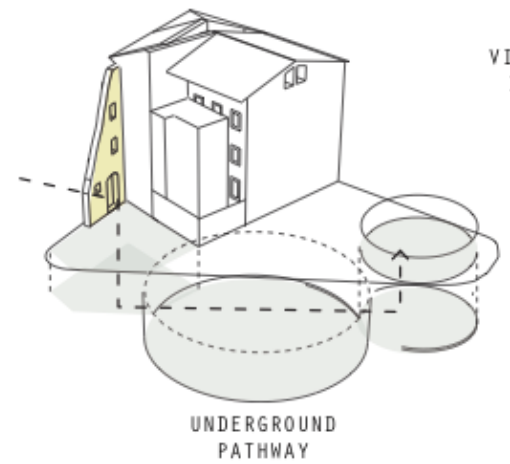

[3] The chapel is organized by programmatically from rooms of private prayer underground to a main oral prayer space at the top. The Chapel opens to the town square, and glows at night, hovering above the city ground-hidden, withdrawn, and asks to maintain its mystery. 


\section{DESIGN LEVEL}

Designing at the level of the city

Looking at the generative

power of city and site

At the level of the body

We always searching for the

horizon. Connecting movement

of the head-on-the-body with

what is percived in VR from a

central POV.
SCALES IN VR

[1] Narrative

[2] Immersive Scale

At the level of the building

Relating a building's parts to

[3] Building scale (Scale of Fitness)

its whole and to each other

At the level of the spiritual

Exploring a sense of wonder, a sense of awe, the smallness of

[4] Relational (Sensory) scale

what we are, or even Bachelard's

'intimite immensity' 
Digital space to digital place

Sense of narrative

procession/sequence

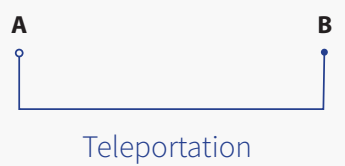

TO TERRACE

Teleportation

Entrance

CAFÉ

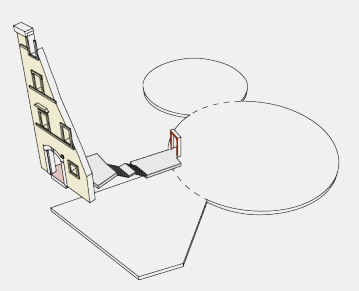

Sense of place

Sense of context

(orientation)

Subterranean Exhibition Gallery

INT. EXHIBITION ROOM

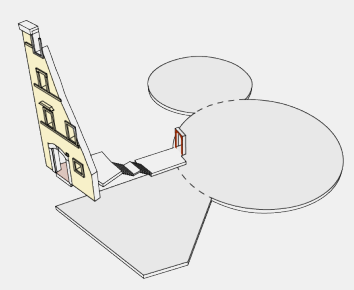

Sense of space

Sense of experience:

MAJOR SPACE

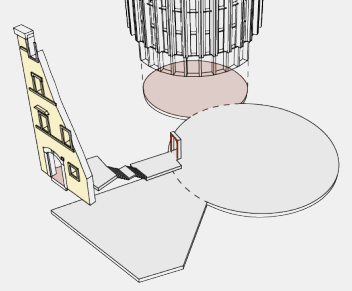

ineffability, awe, wonder,

immensity 


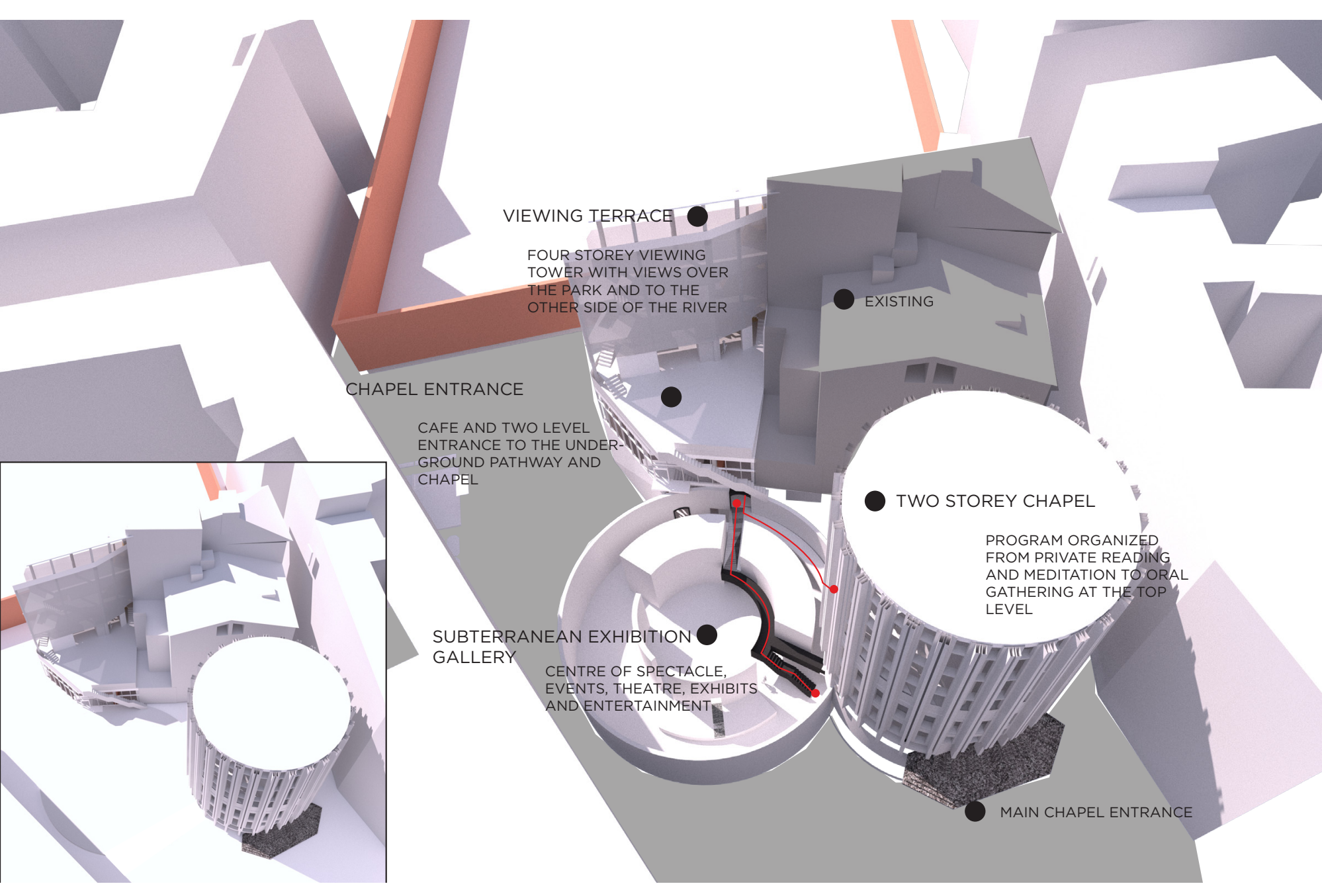

Figure 7.3 Design Axo 


\section{Design Workflow}

A building process is a sequence of decisions. Any design process can be broken up into a methodology that varies from designer to designer. The overall workflow follows a process from thought to evaluation:

Intelligence $\rightarrow$ Design $\rightarrow$ Choice $\rightarrow$ Implementation $\rightarrow$ Evaluation $^{1}$

The "typical product" development cycle can be broken up into a feedback loop from concept to iteration.

\section{Concept $\rightarrow$ Design $\rightarrow$ Evaluation $\rightarrow$ Redesign}

Introduing FPRs into the design stage does not change the process but adds a level of sensitivity and sensibility to the experiential qualities of architectural spaces so that the designer can evaluate not only the geometry and form, but also the look and feel provided by the impression collected in immersion.

$$
\text { Concept } \rightarrow \begin{gathered}
\text { Design and experience in } \\
\text { immersion for evaluation }
\end{gathered} \rightarrow \text { Redesign }
$$

ENDNOTES

1 Lang, Jon T. Designing for Human Behavior Architecture and the Behavioral Sciences. Dowden, Hutchinson \& Ross, 1980, 228 


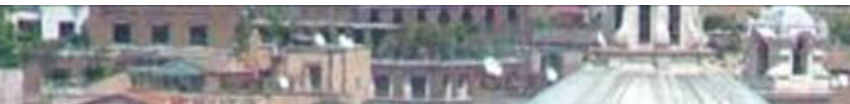

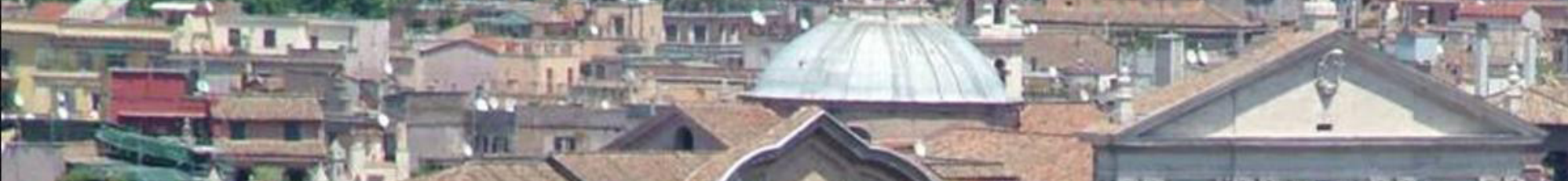

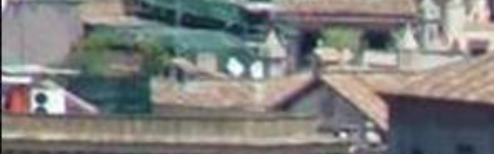

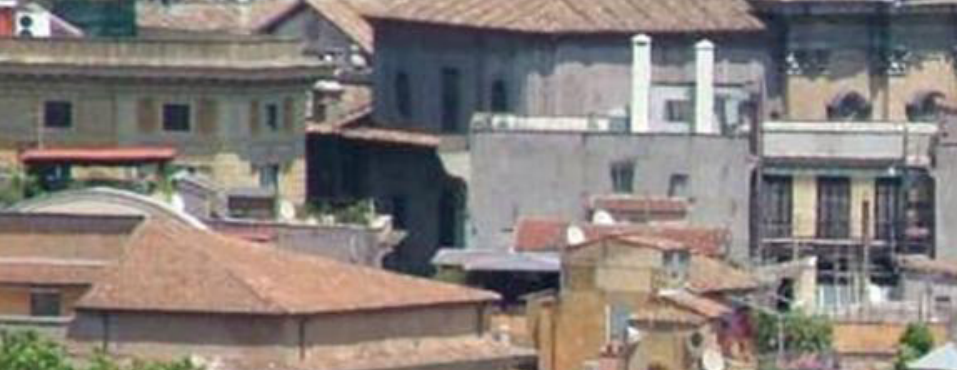

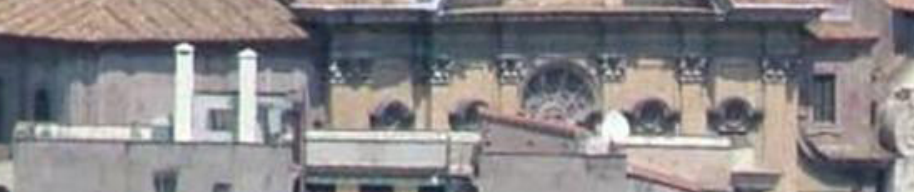

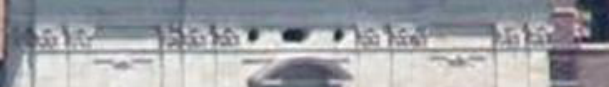
(x)

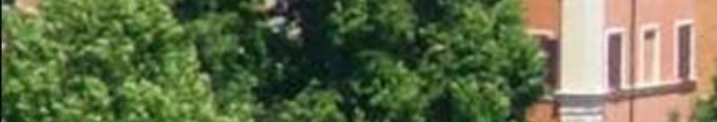

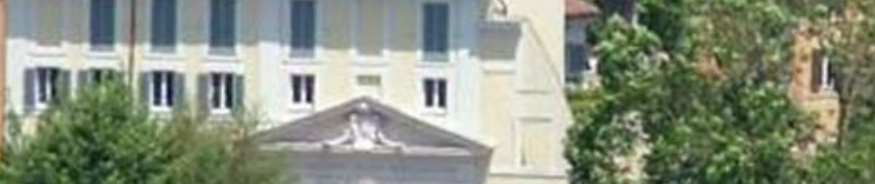

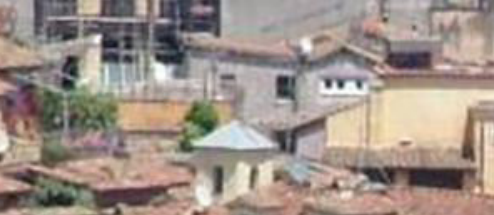
3.

at- -7 . 2. 6it

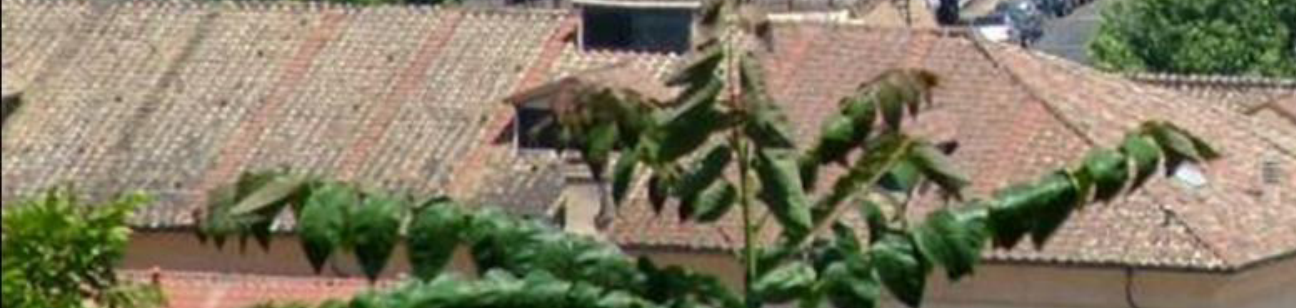

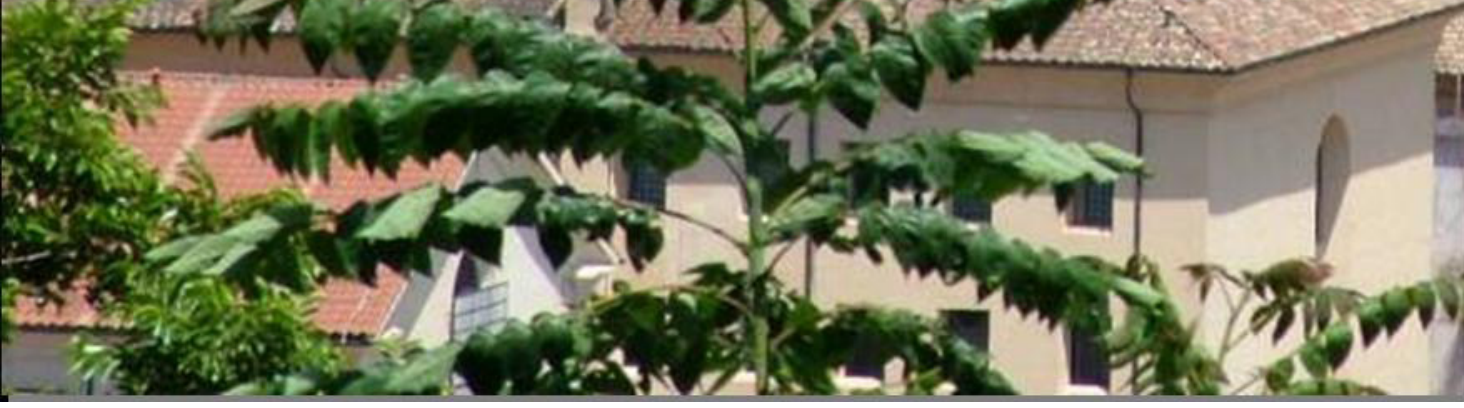
$\frac{10}{10}$

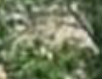




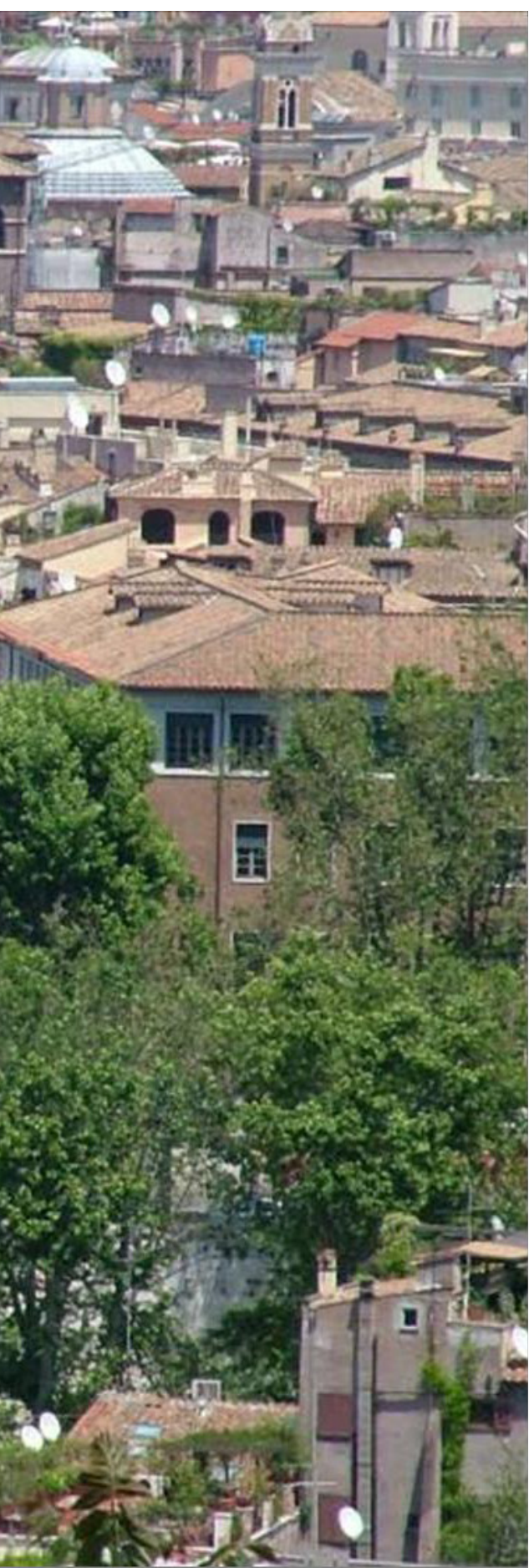

\section{Narrative Scale:} Creation of experience

"...our contemporary environment lacks beauty or the proper human scale and balance of the res publica and res private, but it also lacks the humanizing effects of meaning and empathy, issues that ennoble not only our public life but our public soul." -Spiro Kostoff, The City Assembled

\section{The First Level of Expression}

The first level of expression of impressionable spaces is their narrative. Once we know the story behind something, we understand and connect with it better. VR links our understanding with the ability to know through user experience.

The narrative is fundamental to all four design levels outlined in this section. In VR, the scale acts at the level of the city by engaging all the design intentions in a conscious sequential story. The design intentions are formulated from the site visit, an investigation into the area's historic development. The possibilities provided by VR in design aim:

[1] To revitalize the public square in order to generate greater interaction between inhabitants and visitors

[2] To repurpose the existing wall ruin, and knit it and new architecture into the existing context

[3] To consider the 360-degree of the visual experience of place beyond what fits into a render frame

Figure 8.1 Rione Regalo photographed from above. Centered is the San Filippo Neri church facade and wall ruin on the site of intervention.
[4] To create a narrative experience that questions where we 'fit' at various scales, in attempt to challenge human scale and perception 

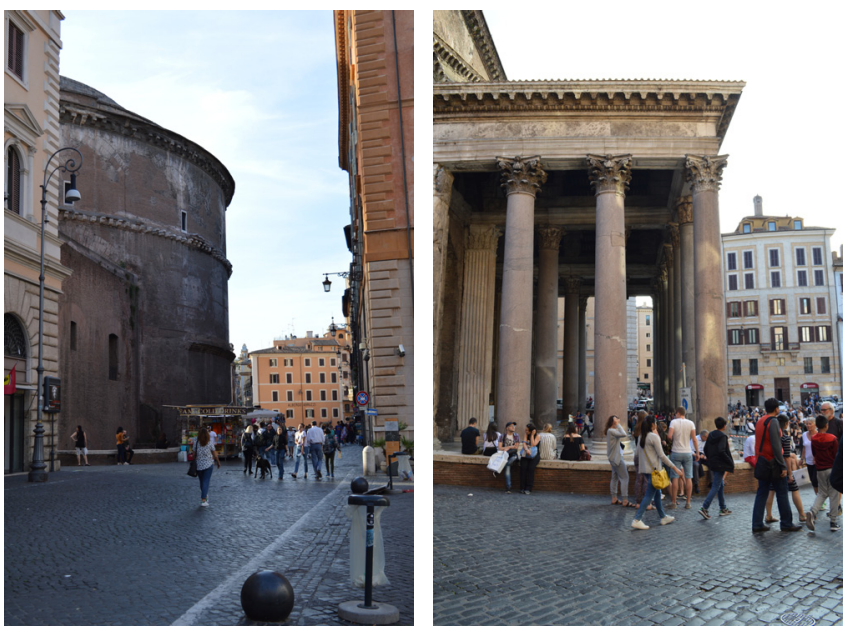

Figure 8.2-8.3 Walking up to the Pantheon entrance.

\section{The Generative Power of City and Site}

Design processes include an interpretive response to site conditions especially when rooted in a pre-existing historical context. There is an inherent value to site and existing environmental conditions that is often neglected in contemporary architecture. Rome is a case in point. It is a city built on layers, and repurposed spoils, designed for the human scale. For Rome, it is the existing mnemonic model that structures the experience and image of the city through its urban fabric. Author of Timeless Cities, David Mayernik examines the experiential links of the city:

To the humanist mind cities needed continuity with their past, between buildings along a street or around a piazza, even across a river, to be harmonious like the universe and memorable like the cities of our imagination. (Mayernik 58)

The ancient conditions of over two thousand years, along with the rise of the Renaissance have created an overarching 'mental bridge' across the city. In Rome, where "the ancient is a contemporary, everyday event" ${ }^{1}$, the city life creates encounters and conditions of experience from which a new design can cultivate. Mayernik explains the evolution of Rome by describing the city as a giant mnemonic device:

Essentially the classic mnemotechnic used visual imagery - figures, buildings, and cities - as cues to help remember words and abstract concepts... Without the printing press, the ancient world put a high value on personal memory skills; one could in effect carry a library in one's head... [E]ssentially one invented a mental architecture in the act of remembering a text that one mentally "revisited" when it was necessary later to recall the text...Conversely, when the Hellenistic architect thought about buildings and cities, he imagined them containing memories of ideas. Thus some of the same rules that governed the making of imaginary memory cities for the rhetorician also informed the architect's process of making real cities memorable. (Mayernik 58)

He calls this process, "mnemotectonics'. Essentially, what linked the city together in a narrative structure were Rome's public processions. In the 16 century, when Rome's city fabric was still a series of fragments, the theme of the procession transformed the city into a 'teatrum mundi'-a grand theatre of the world. The focus of this narrative experience was for the Queen and pilgrims, beginning at the Northern gate for the travellers from northern Europe. The chosen route initiated the building of temporary structures and Rome's grandest masterpieces.

Rome had not always valued perservation. The Renaissance destroyed, lauded and re-used the ruins of ancient Rome for its own needs. And while the city has a 'finished text that is continually interpreted in the reading of those who govern it'2 that is founded in the tourism economy and popular culture, the city embraces its vast historical narrative to reflect in the modern day. The city grows and evolves, affected by the fashion and tastes of every age. The Roman position was to maintain and build upon 
both poetically and physically on what was. The churches built in the centuries between the collapse and the Renaissance, imitated the ancient pagan basilicas and the Constantinian churches, most Roman buildings were used as foundations, armatures or even quarries for raw materials. The Renaissance, as an intellectual rebirth of the ideals and ideas of classical culture, "marked a sea of change in this crudely cannibalistic attitude toward the past". Novelist and journalist, James Howard Kunstler, claims that,

The devices in civic design that had adorned Europe-derived chiefly from the notion that the space between the buildings was as important as the buildings themselves. ${ }^{3}$

Rome has a number of political and religious foci dispersed throughout the city, such as the papacy, sites of Christian martyrdom, ancient monuments, papal family residences, and the Vatican. Michelangelo's Piazza del Campidoglio (Capitoline Hill) opens toward the Palazzo Senatorio, the symbolic political head of the city, to a trapezoidal piazza. Piazzas are essential learning ground for the Roman context. Many piazza open towards their churches, where communal life can foster and spill out from the churches. The open participation of the piazzas converges and unites the many building facades surrounded by it. (See Figure 7.7 Largo dei Fiorentini, the church located north on Via Giulia)

The construction of churches was very important to fulfill the liturgical functions, inspired and motivated to express the architectural style of the period. Rome has more than 900 churches, each one of them contributing something different to Rome's 'mental bridge'. The via Giulia chapel project aims to elevate the human being to connect with the interior and to reflect on one's awareness and existence.

The sixteenth century sought the grandest urban planning ambitions projected onto the edges of inhabited built-up areas, addressed to visitors, such as pilgrims and dignitaries, than to the Roman citizens. ${ }^{4}$ One of the projects was the straightening of two street: The via Giulia and via della Lungara (point towards St.Peter's). The project began in 1508, as part of Julius' urbanistic project to renovation/re-organization of the medieval city of Rome in an effort to strengthen its unrealized assets to match the city as it grew in economic importance. Via Giulia was proposed as an artery to connect all government institutions-the Palazzo della Cancelleria (nearing completion at the time), the papal mint, and the Palazzo dei Tribunali. Also linked the river port of Ripa Grande with Via della Lungara and to Ponte Sisto. This enabled secure and convenient merchandise transport to the heart of the marketing and banking zone. The task of laying out the street was given to Donato Bramante, who was in charge of the Basilica of Saint Peter at the time, where 'all the offices and seats of power of Rome [where] in one place'.5 Julius' project was never fully realized, and Via Giulia developed into an area of modest houses with gardens behind built for private owners, aslso known as the "houses of Raphael" ${ }^{6}$ composed of ground-floor shop fronts.

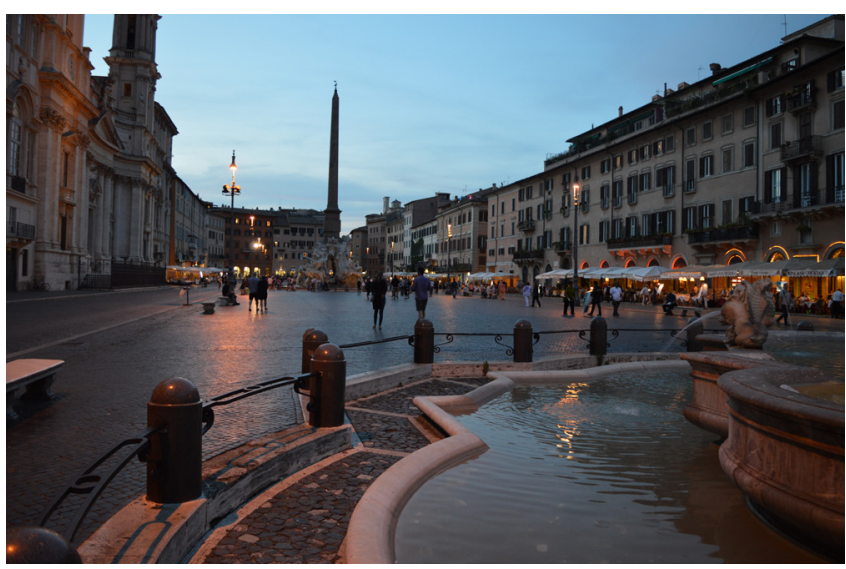

Figure 7.4 Piazza Navona. 


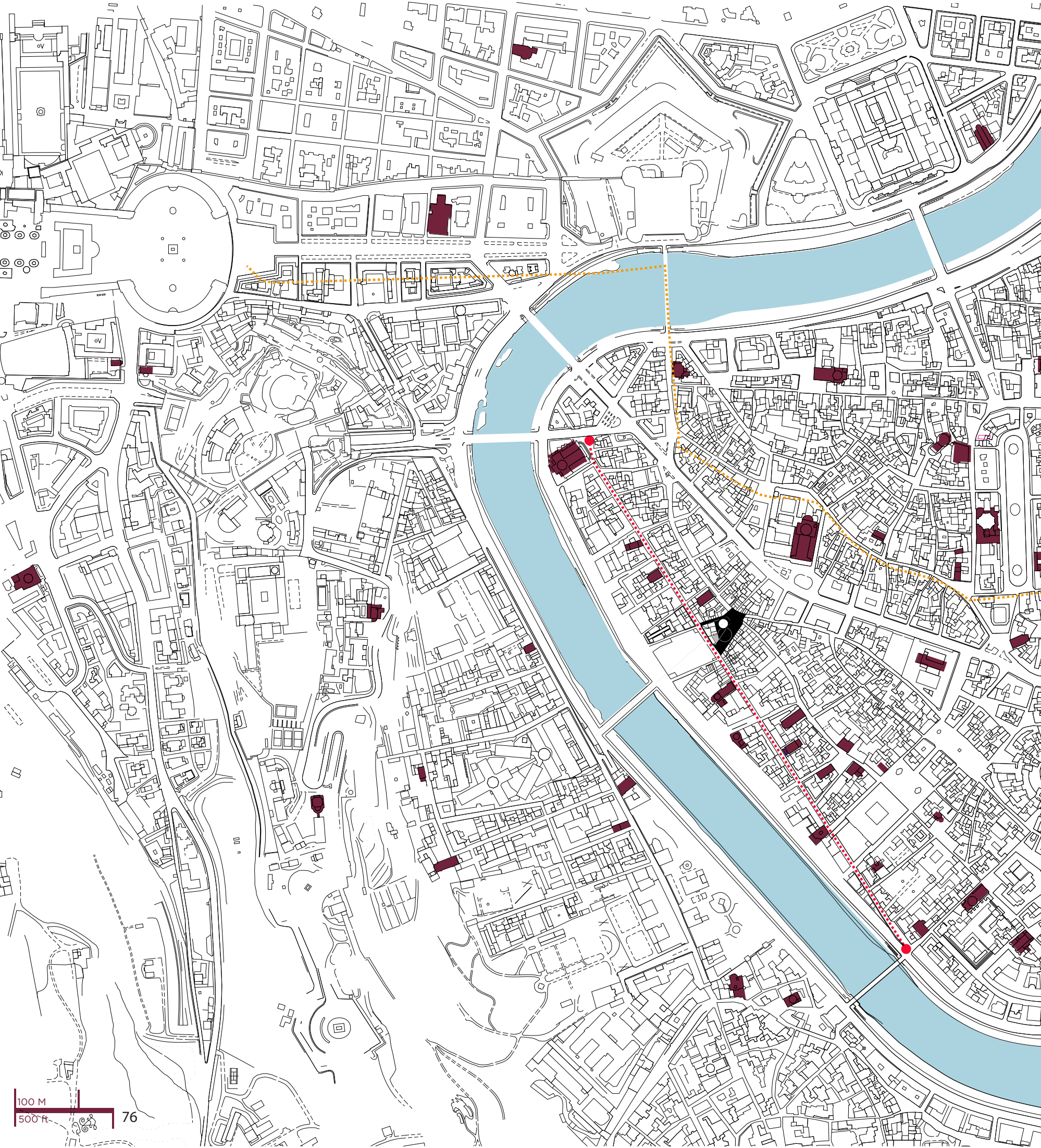


Via Giulia is located in the rione Regola, in the historic center of Rome, originating from one of the first important urban planning projects in Renaissance Rome. Via Giulia is the first modern road in Europe suggestive of the Renaissance urban revolution. ${ }^{6}$ For centuries, via Giulia and via della Lungara have been meeting places for different nationalities. Originally part of Julius II's program for reorganizing Rome, (credited to Bramante's influence), had been only partially carried out. It is the first European example of urban renewal since Antiquity-as project promised to 'pierce through the heart of Rome.7 Via Giulia runs from Ponte Sisto to the church of San Giovanni del Fiorentini following the curve of the Tiber. In the 16th century Via Giulia became one of the most fashionable streets for the borghesi and the Florentine community. Today, it is part of Rome's most elite shopping street and part of a Residential neighborhood forming a straight line, one kilometer long. Via Giulia was named after Pope Julius II (nephew of Sixtus IV) who took it upon himself to continue his uncle's projects, creating a network of radiating streets that link the seven major pilgrimage churches. It was this sequence that created a personal, spiritual narrative for Rome.

\section{Existing}

San Filippo Neri a Via Giulia is a small, 18th century confraternity and conventual church. The building complex was built in 1603, through the patronage of a devout glover from Florence. There was a small oratory next to the church, a hospice for infirm priests and institution for girls who could not afford a dowry, later run by a small religious sisterhood. It was rebuilt in 1768, designed by Giovanni Francesco Fiori, and rededicated to St. Philip (nicknamed Little St Philip). In 1853, the church and orator were damaged when the Tiber flooded. The interior was restored under the patronage of Pope Pius IX. Due to the number of churches in the area, the church fell into disrepair in the early 20th century. The site is a casualty of Mussolini's plans for new road in the city center in 1940 (the road from Ponte Mazzini and the Corso Vittorio Emanule). The church was deconsecrated, partly demolished, and adapted into an apartment block. The complex housed a market warehouse until the 1960s (when the building became derelict). In 1993, the church was commissioned to be renovated, but was never finished due to lack of funding. The façade was restored in 2000, preserving the façade inscription: DEO IN HONOREM'S PHILIPPI NERII DICATUM. ${ }^{7}$ The small oratory is not longer on the site. Part of the wall facade remains on the site with an arched entrance and 5 window openings that lay at an acute angle to the street.

\section{Examining the metaphor of the city as a narrative}

It is important to remember the mnemonic narrative that guides the context, eloquently described by Mayernik:

Reading them [cities and books] was a dynamic and participatory act, and a city's narrative content was continually reborn for the alert citizen. "Building" the city in its fullest sense was therefore a continuous process rather than a onetime act, and the meanings cities embodied were placed there and actively retrieved in a continual exchange. Keeping cities alive is an ongoing operation of building even more than preservation-because the latter is ultimately, tragically, a pessimistic resignation to our inability to equal the past. (Mayernik, 232)

Each design principle establishes conditions for the existing site, 

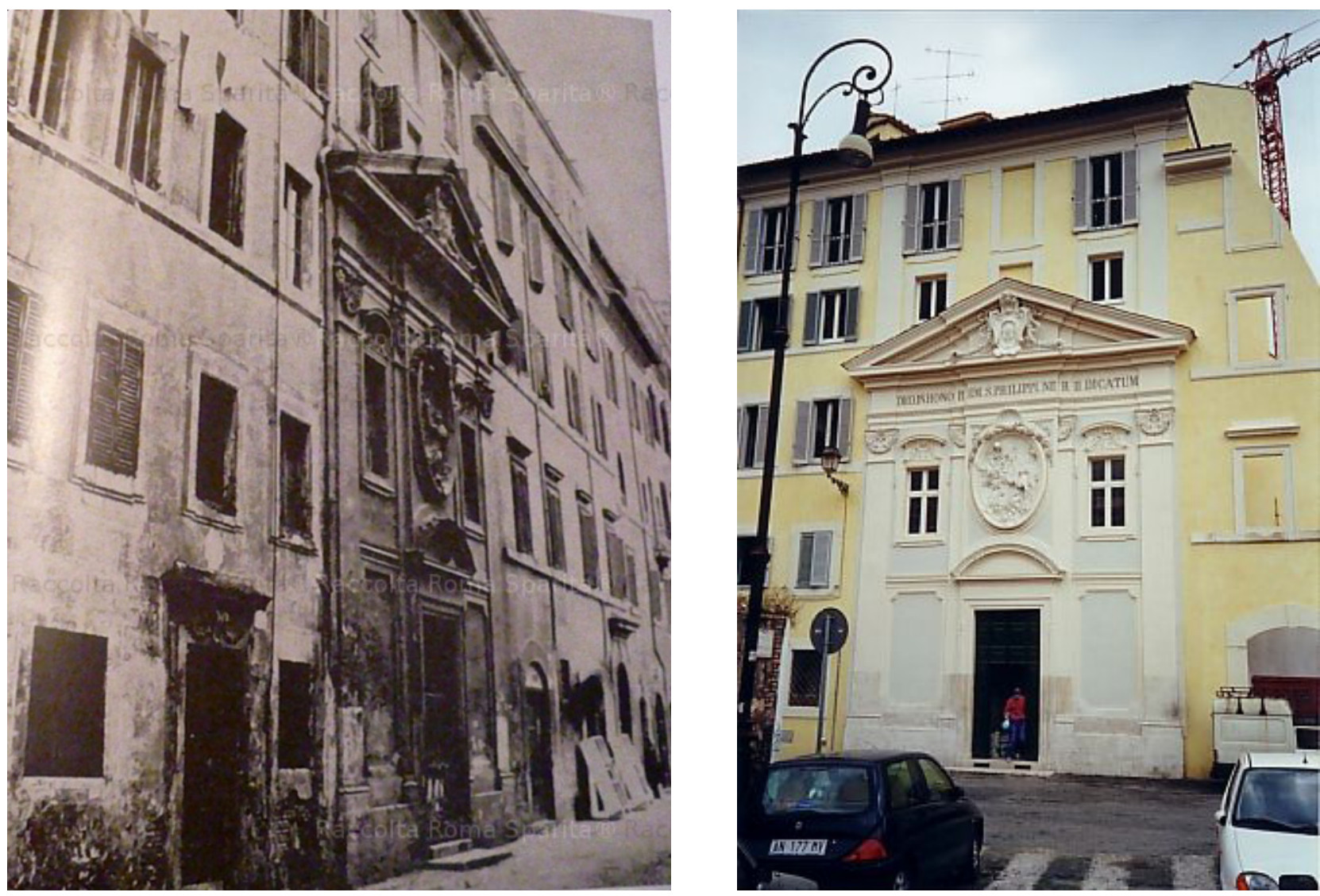

Figure 8.9 [LEFT] Entrance to contiguous

18th century church facade, San Filippo

Neri before demolition Figure 8.10 [RIGHT]

Church facade after restoration in 1993 

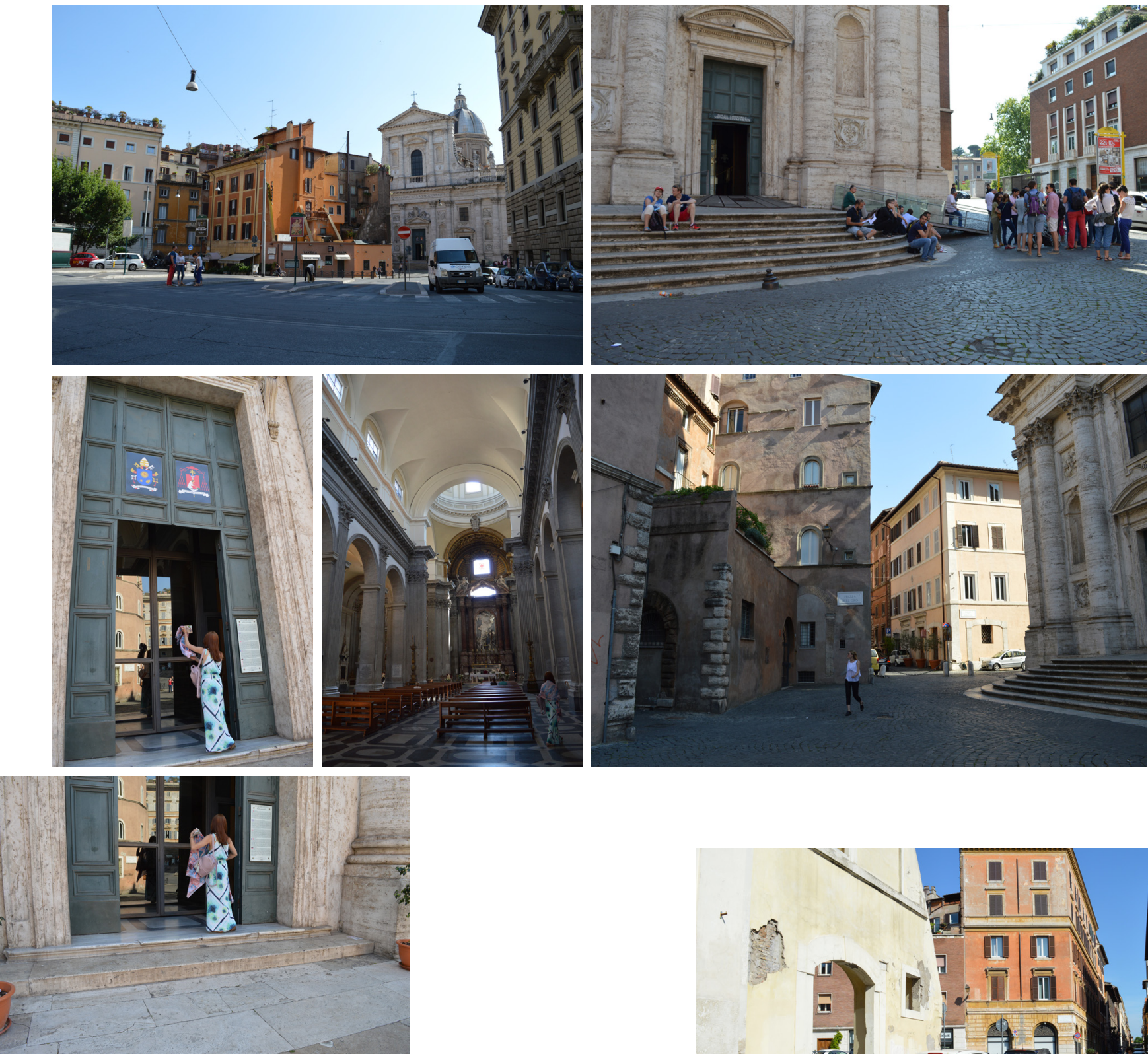

Figure 8.11-8.12 [ABOVE] San Giovanni

dei Florentini Church at the North end of

via Giulia. Figure 8.13-8.14 [LEFT] Woman

covers her head as she enters. Figure $\mathbf{8 . 1 5}$

[CENTER] San Giovanni dei Florentini Church

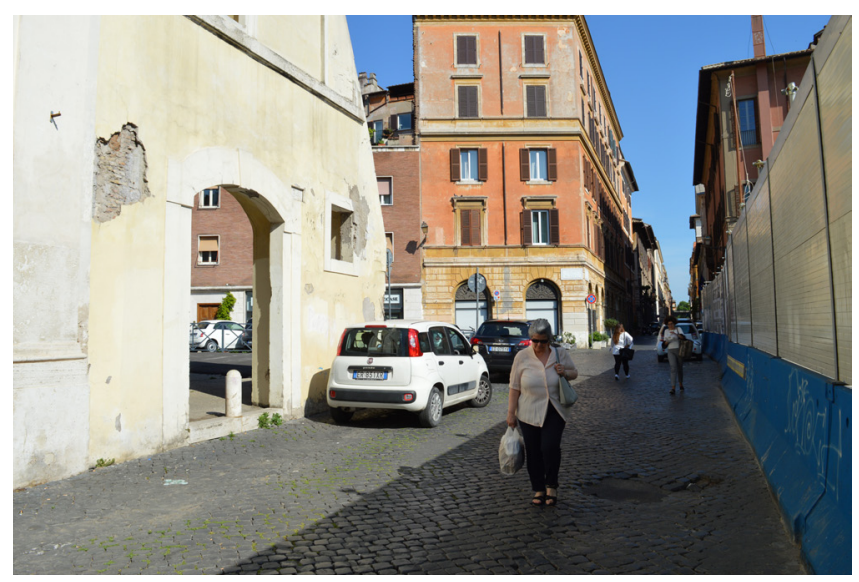

interior 8.16 [RIGHT] San Giovanni dei

Florentini Church steps and entrance to via

Giulia 

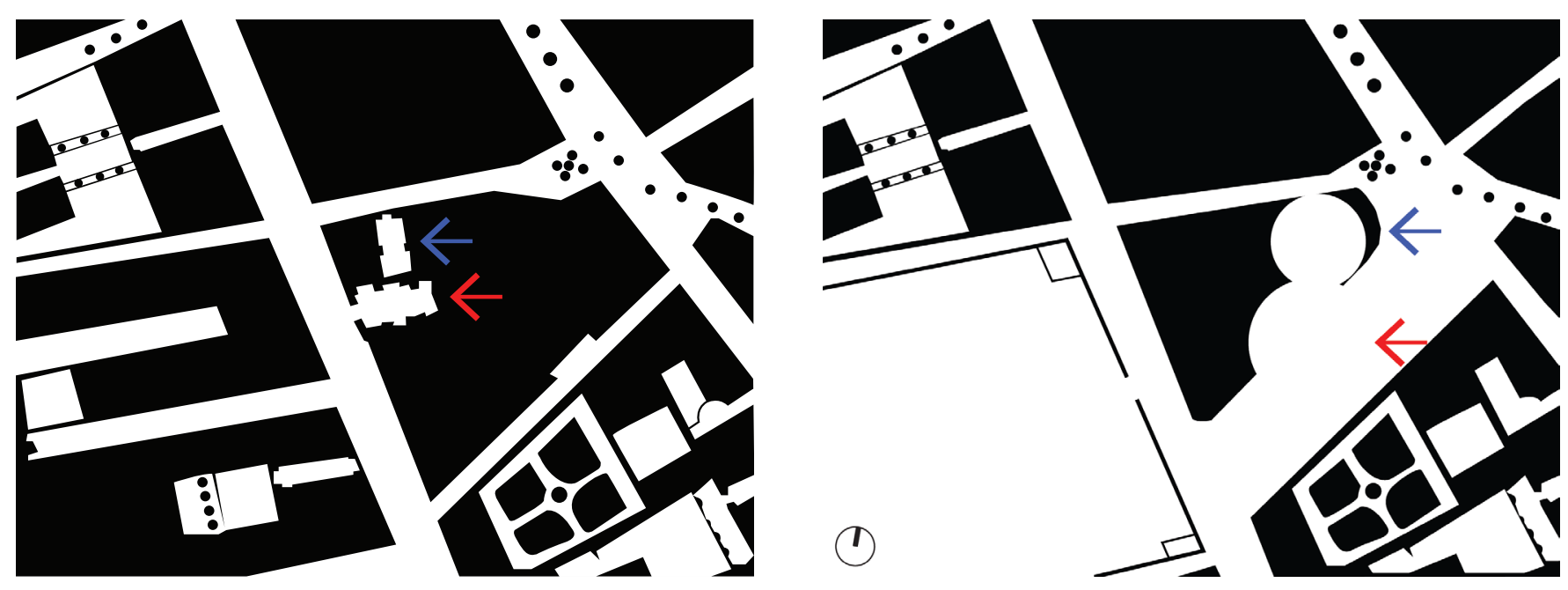

Figure 8.23 [LEFT] Iconic map of Rome produced by Giambattista Nolli in 1748 depicting the figureground condition of Rome rendering the interior of public buildings numbering each building (Roman Ruins and Ruin redevelopment. 1320 sites, 60 labeled as ruins.) San Filippo Neri in Via Giulia (\#666) and Oratorio delle Piaghe di Gesù Cristo (\#667) Figure 8.24 [RIGHT] New design in figure-ground plan.
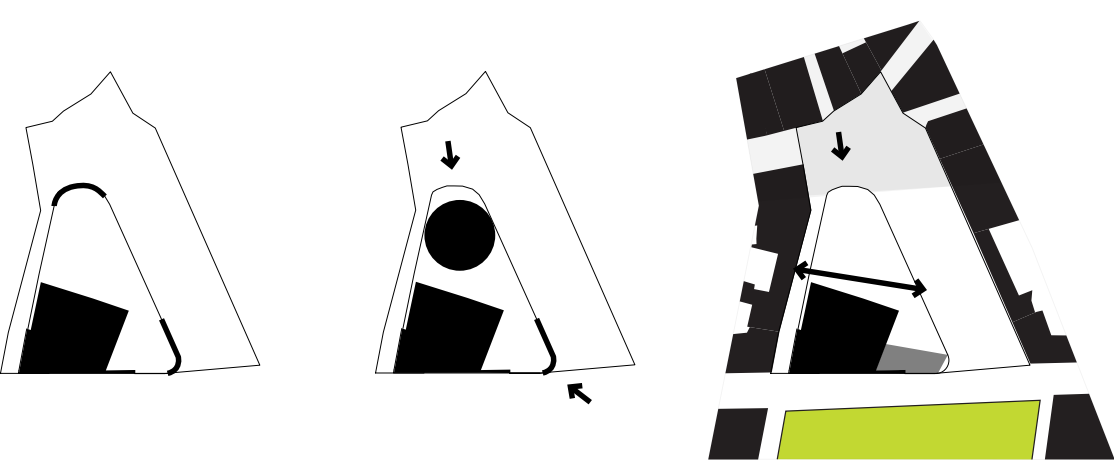

$\bigcirc$

Figure 8.25 Site piazza parti 
informed by the current reality of Rome's ancient context. The narrative, immersive, building and relational scales in VR address the design intentions of the project.

[Design Intension 1] To revitalize the public square in order to generate greater interaction between inhabitants and visitors

The site is one of the largest open-air voids of Rome. In an effort to maximize and revitalize the public square and intervention area, it is important to address the site block as a whole and reassess its boundary conditions to the existing context. By designing a spatial sequence that weaves an experience from the external to the internal, the chapel experience will aim to engage perceiver through their perception and sensations.

Rome's city blocks are most evident in plan, even more notorious for Giambattista Nolli's 1748 figure-ground map [Figure 8.2], and while the eye can perceive patterns of the figure-ground conditions easier in plan, when viewed stereoscopically, the spatial conditions of proximity, distance/depth, and privacy emerge within our perception of figure-ground to occupying volumetric solidvoid.

The mnemonic model of the site's history (the beingthere) is reflected in the modeled site fractal within the VR views. Designing a place for discussion and study, the experience is oriented towards a progression of space from secular to private reading to oral practice. A part of the existing wall left over from demolition is integrated into the new design to allow for an interesting narrative composition for the new intervention. The existing wall is at an acute angle to the street frontage.

\section{VR Narrative}

The role of narration is heavily skewed in VR. We hold the power to observe what we want, but we also give that power away, as we have no control of what another navigator will look at. VR video breaks the fourth wall of narration. Utimately, we are looking at what can and cannot be 'concealed'.

Nevertheless, teleportation allows us to experience design in sequence and navigate through views by providing a conscious flow of space guided by thresholds, doorways and openings. The viewer can develop a sense of narrative, procession and sequence of space, while assessing their interconnection and visual navigation. The engagement of the next three scales will be perceived in a procession that takes one through the design project in order of design conception. The narrative guides the perception of the environment, the building elements, and the viewer's perception of the virtual space. Through teleportation, the viewer can develop a sense of narrative, procession and sequence of space.
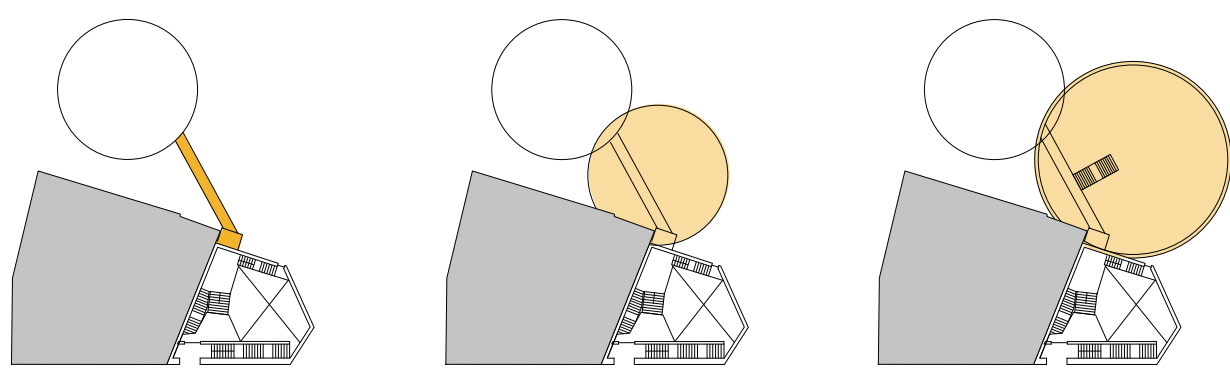

Figure 8.26 [LEFT] Design parti of the threshold between the entrance and the new chapel building. 

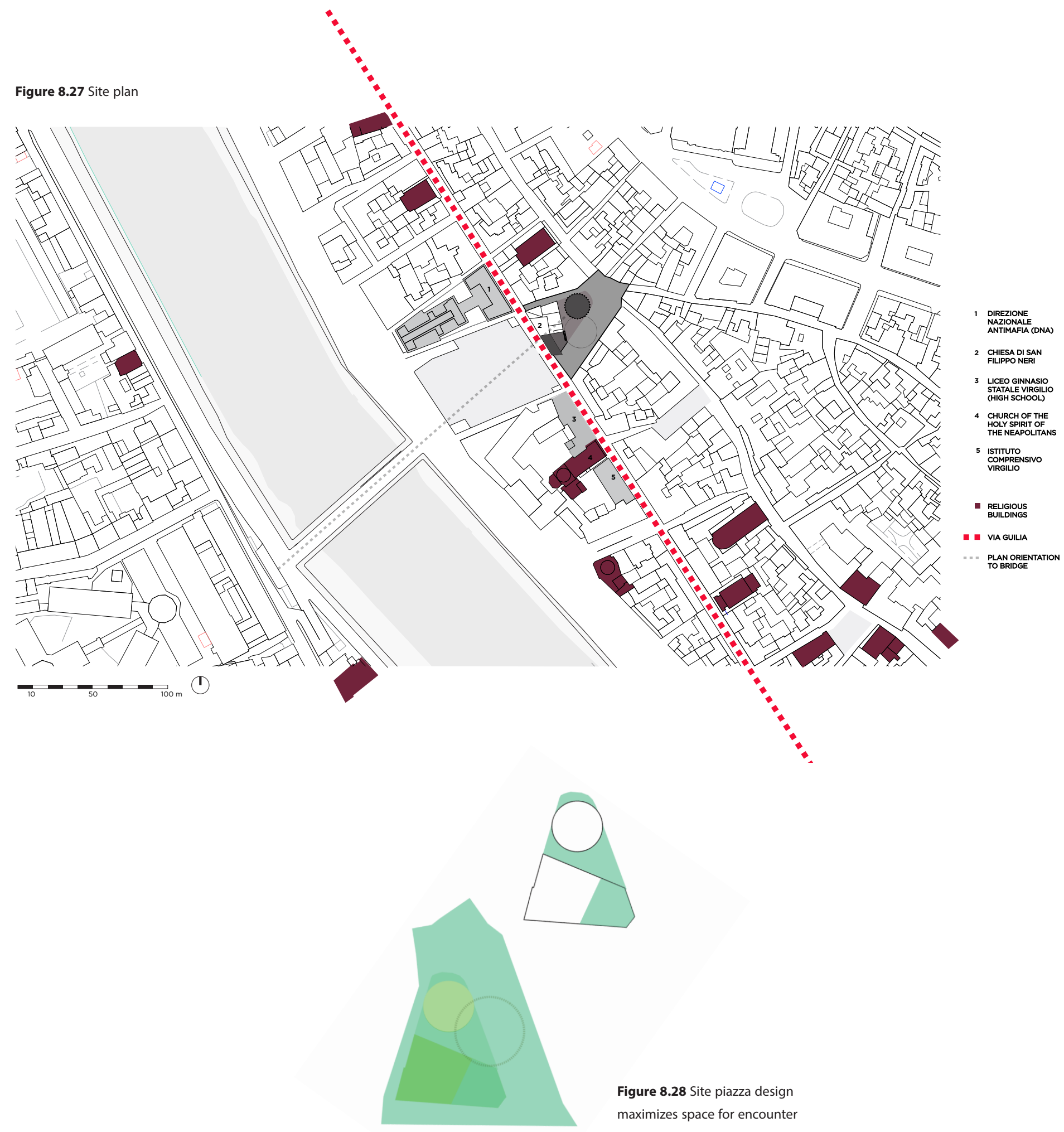

Figure 8.28 Site piazza design

maximizes space for encounter 


\section{VR teleportation: Space A to Space B to Space C}

VR iterative process/procedure: Entrance to threshold to patio; the spatial conditions of proximity, distance/depth, and privacy emerge within our perception of figure-ground and solid-void.

Verbal to visual to spatial operations (translate to program order)

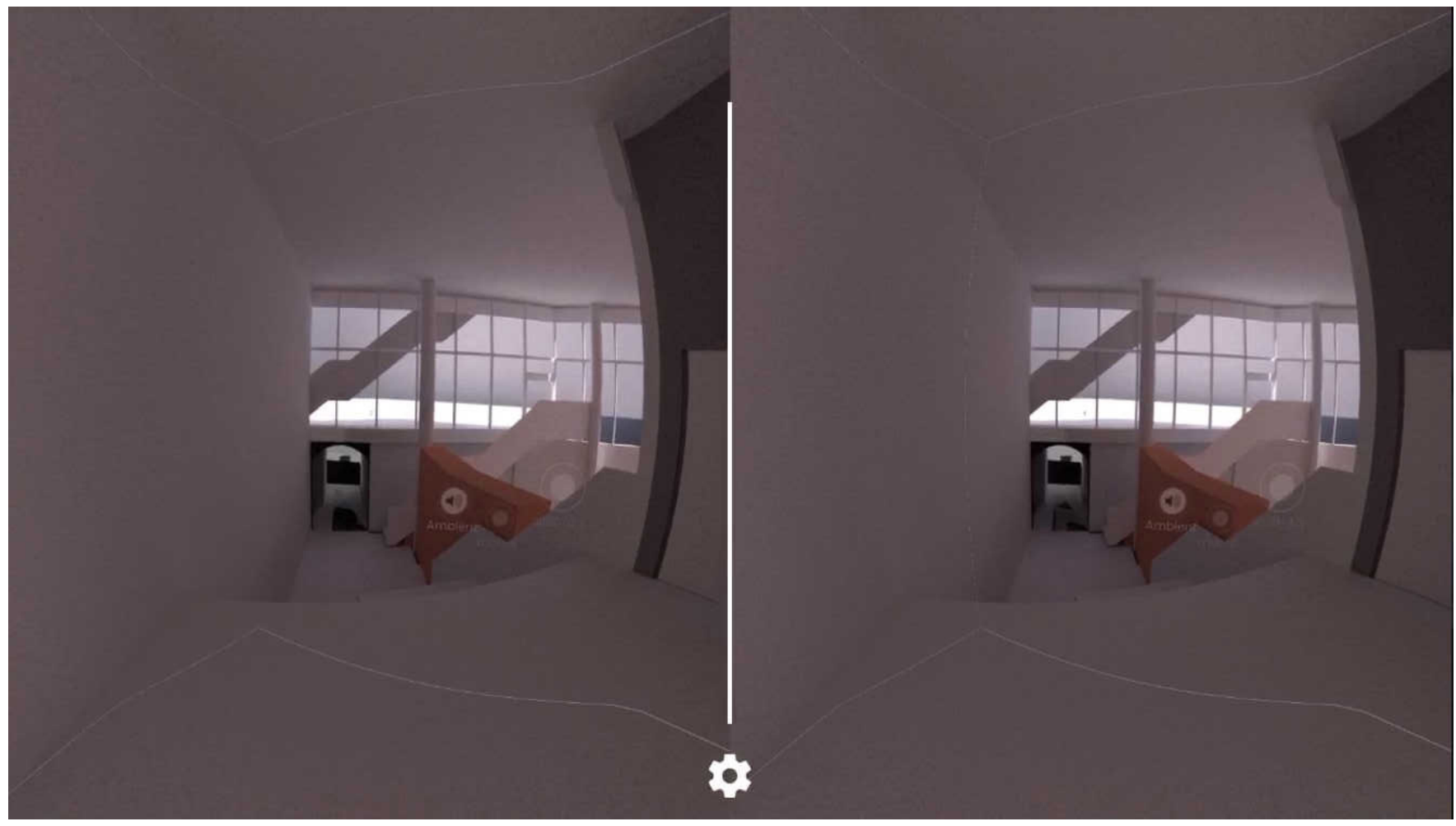

Figure 8.29 Video Capture 1

\section{[ Entrance to Café interior to Terrace]}

Teleportation is used to experience the narrative. 


\section{ENDNOTES}

1 Brandolini, Sebastiano. Rome: New

Architecture. Skira, 2008, p11.

2 ibid.

3 Kunstler, James Howard. The Geography

of Nowhere: The Rise and Decline of America's Man-

Made Landscape, Simon and Schuster, 1994, p39.

$4 \quad$ Mayernik 82-83.

5 ibid

6 ibid

7 San Filippo Neri in Via Giulia, romafelix.com/ sfilineri.htm. 


\section{Immersive Scale: Environment to be perceived}

\section{Immersive Scale examined at the level of the body}

Immersive scale characterizes a sense of place, orientation, and how the building fits within an environment and/or context. In this case, the virtual context is a fractal site of Rome, Italy, where the site specificity of the neighboring buildings and the preserved wall element act as points of reference to the user-navigator for orientation and a sense of place.

The immersive quality of context is removed in 3D modeling. The digital model does not translate scale, leaving the user to experience the model from the 'outside'. The immersive
"Everything a man does is associated with the experience of space. Nothing occurs, real or imagined, without a spatial context, because space (along with time) is one of the principles organizing systems for living organisms. Proxemics deals with man's use and structuring of space, particularly the unconscious patterns that deeply influence life." -Walter Gropius ${ }^{1}$

quality of spatially in VR sets up perceptual engagement with the existing context by building a strong mental map of the inhabited space. For the designer, early exposure to spatial experience aims to create a contextual 'sense of place.' VR allows us to conjecture the sense of scale that is mediated by VR to accomplish an (end) experience, responding to the second design intention:

[Design Intension 2] To repurpose the existing wall ruin, and knit it and new architecture into the existing context 
In this case, the context is a fractal site of Rome, Italy, where the site specificity of the neighboring buildings and the preserved wall element act as points of reference to the user-navigator for orientation

Context is removed in 3D modeling. The digital model does not translate scale, leaving the user to experience the model from the 'outside'. The immersive quality of spatially in VR sets up perceptual engagement with the existing context by building a strong mental map of the inhabited space. For the designer, early exposure to spatial experience aims to create a contextual 'sense of place'.

Architectural historian and theorist Dalibor Vesely expresses two key points for architectural design. The first is to know and understand the real possibilities of a particular design task (based on the hermeneutical articulation of the world) and the second is the moment architecture creates a dialogue with existing conditions. This is where designers can make a contribution to support different experiences, different way of life, even create a different way of thinking about the nature of our experiences.

We begin to understand place as we explore it, and add value to it. Places cannot be displaced, whereas objects can be. Humans and animals orient to habitat or place, such as landmarks or open spaces.

When the vistas have been put in order by exploratory locomotion, the invariant structure of the house, the town, or the whole habitat will be apprehended. The hidden and the unhidden become one environment. It is not so much as having a bird's-eye view of the terrain as it is being everywhere at once. (Gibson 198-199)
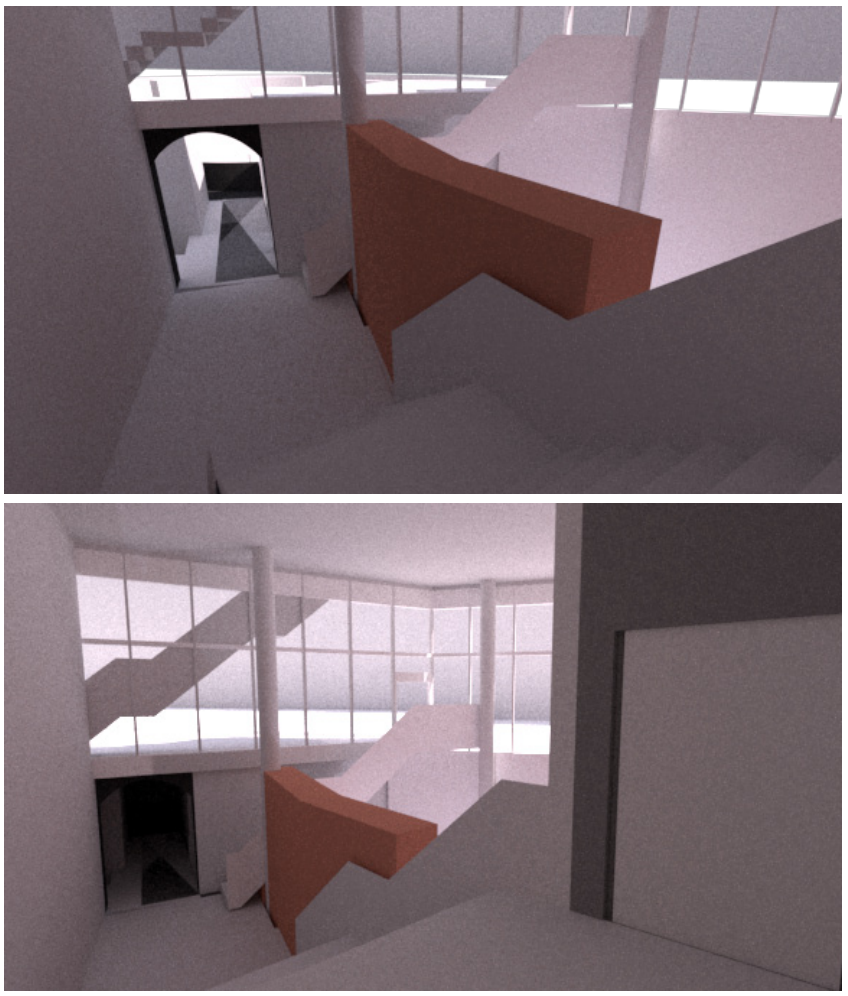

Figure 9.1-9.2 Threshold entrance iterations. Bar indicated in orange.
Figure 9.3-9.8 Stereo Cubemap renderings of the cafe (FPRs) to be viewed as VREs. Thresholds, light and materiality are evaluated and adjusted. Experiemented with materials. 

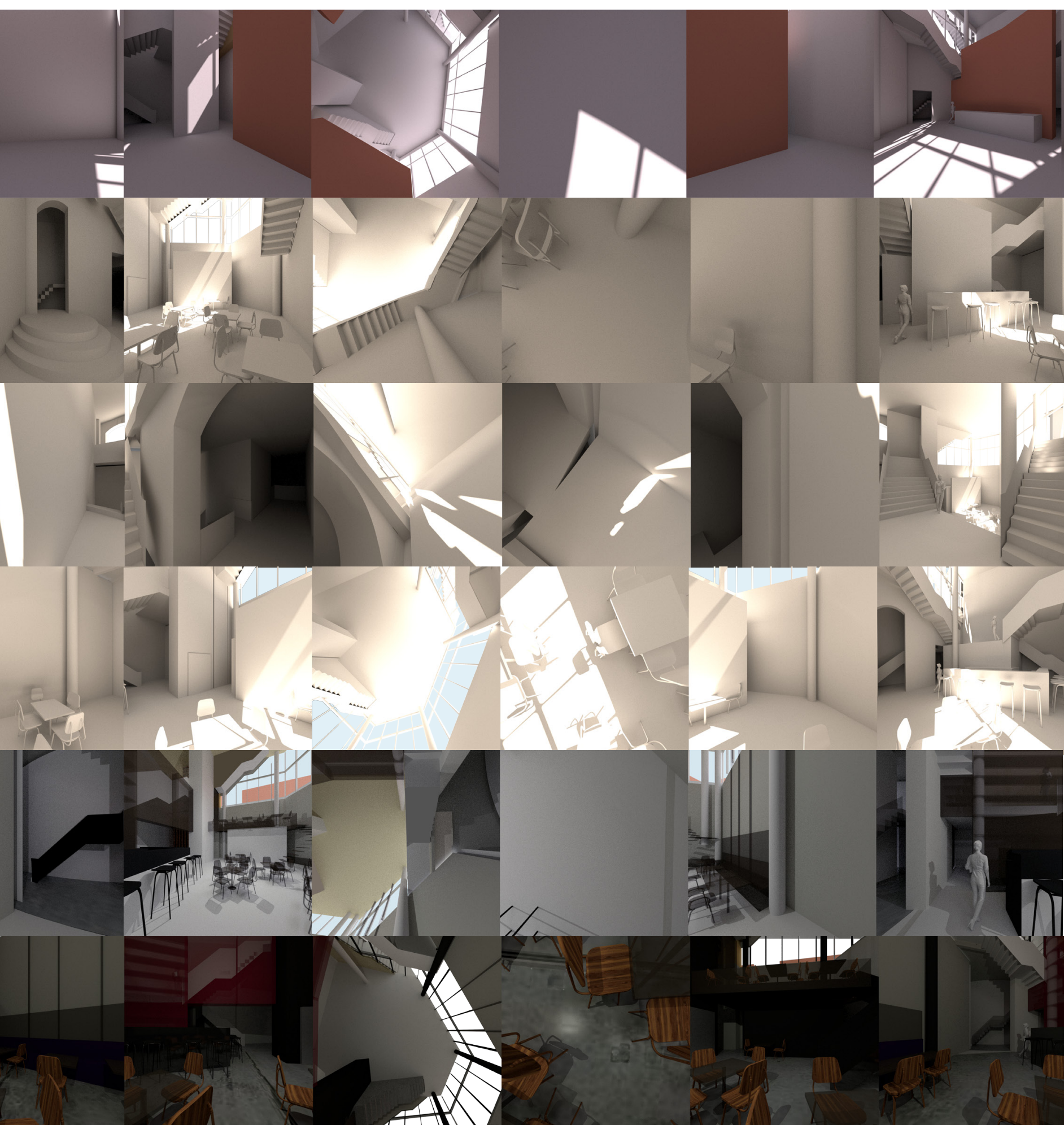
In VR, we can examine appropriately scaled design. This includes: proximity, privacy, and territoriality to be addressed in immersive scale. VR allows us to conjecture the sense of scale in a space to accomplish an (end) experience.

[a] Unrestricted head-on-the-body movement. However, the idea is to stay 'grounded' within the limits/confinements of a human dimension as we continuous search for the horizon.

[b] View selection engages the body at eye level to perceive a 'scalable' concept model in human scale. Activates qualities of apatial intelligence, spatiality, proximity, privacy, spatial boundary, and visual navigation

The topic of immersion cannot be left without mention of fidelity and resolution. There is a great sense of pleasure in experiencing fidelity in the virtual world. For instance, Unity VR achieves impressions of environmental effects such as haze or fog by implementing special distortion material shaping effects into the rendering to enhance fidelity. Given that virtual reality is really 'visual' reality, rather than virtual, Berenson believes, "the work of authentic art stimulates our ideated sensations of touch, and this stimulation is life-enhancing."2 Pallasmaa explains the primacy of vision in the process of experience:

Vision reveals what the touch already knows. We could think of the sense of touch as the unconscious of vision. Our eyes stroke different surfaces, contours and edges, and the unconscious tactile sensation determines the agreeableness or unpleasantness of the experience. The distant and the near are experienced with the same intensity, and they merge 

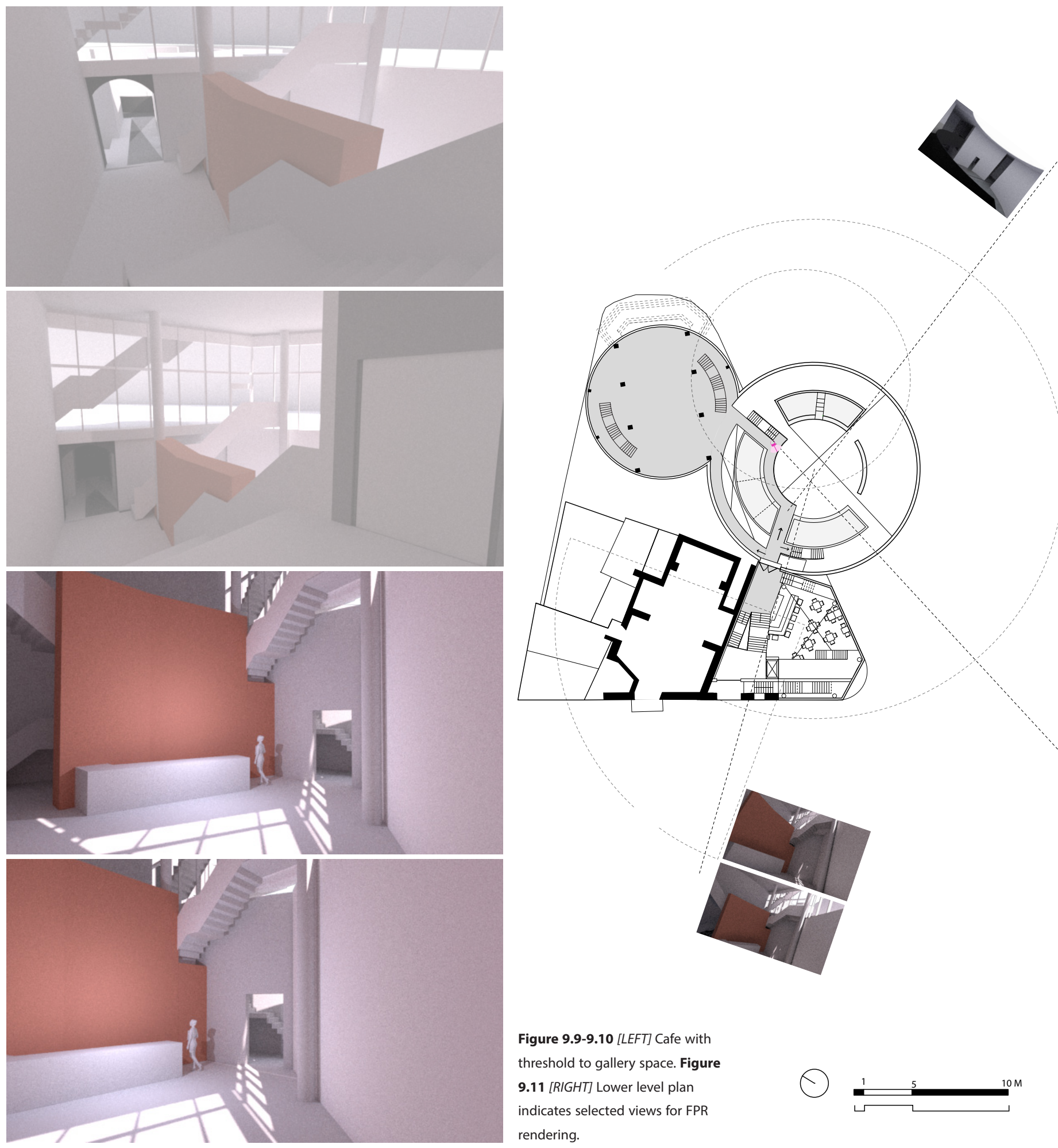

Figure 9.9-9.10 [LEFT] Cafe with threshold to gallery space. Figure 9.11 [RIGHT] Lower level plan indicates selected views for FPR rendering.

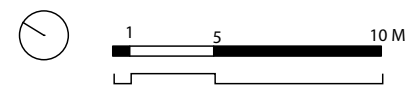


Combining both the sensory and information based perception, there are three factors that we perceive which reflect design thinking in immersive scale:

- Level of abstraction (emphasis on form, massing, and lighting conditions)

- $\quad$ Level of fidelity (modeled fractal context; and high level of colour, texture and materiality that impacts the overall sensory qualities of the environment)

- Level of 'fractal' site/room context (situatedness within exisiting environments and ability to relate to with or within them)

VR provides awareness of designing the 360 of a place, building a strong mental image of the space.

[a] Fractal context model, working with the existing landscape; provides scale

[b] Existing wall fragment provides orientation perceived through scale and colour

The immersive experience of a rendered white massing model in VR, exposes the thinking behind the design process, rendering the project (stereoscopically) present within our view, not as a finished product, but within the process stage. A model with higher level of fidelity and resolution allows the designer to examine and edit finer details in the later stages of the design process. Essentially, the benefit of VR over augmented reality and augmentation is the capability of immersion within the scene as a navigator, rather than the secondary seat of the viewer.
American art historian, Bernard Berenson, suggested that in the process of experiencing art we "imagine a genuine physical encounter through ideated sensations". He states that all art is compounded of sensations, 'of contact, of texture, of weight, of support, of energy, and of union with one's surrounding." In VR, the encounter brings us closer to the understanding of spatial boundaries and cues, such as the gestalt idea of proximity, closedness, as well as privacy and boundless horizon. Most importantly, this occurs at the level of "tactile values". Immersive scale situates the user within the scene of the design and/or its contextual situation. Experience involves sensing weight or mass above and around us.

In the sensation of hearing, touch, sight, and pain we are accustomed to distinguish from among the other elements the element of voluminousness.(134) In the sensation of dizziness or subjective motion, which recent investigation has proved to be connected with stimulation of the semi-circular canals of the ear, the spatial character is very prominent. (James 134)

Pallasmaa argues for a sensory intimacy with spaces for 'sensuous curiosity and pleasure'. He claims that 'to at least some extent every place can be remembered, partly because it is unique, but partly because it has affected our bodies and generated enough associations to hold it in our personal worlds." By engaging in the sensory qualities created by the virtual environment, the designer can become intimately aware of design implications at various scales. 

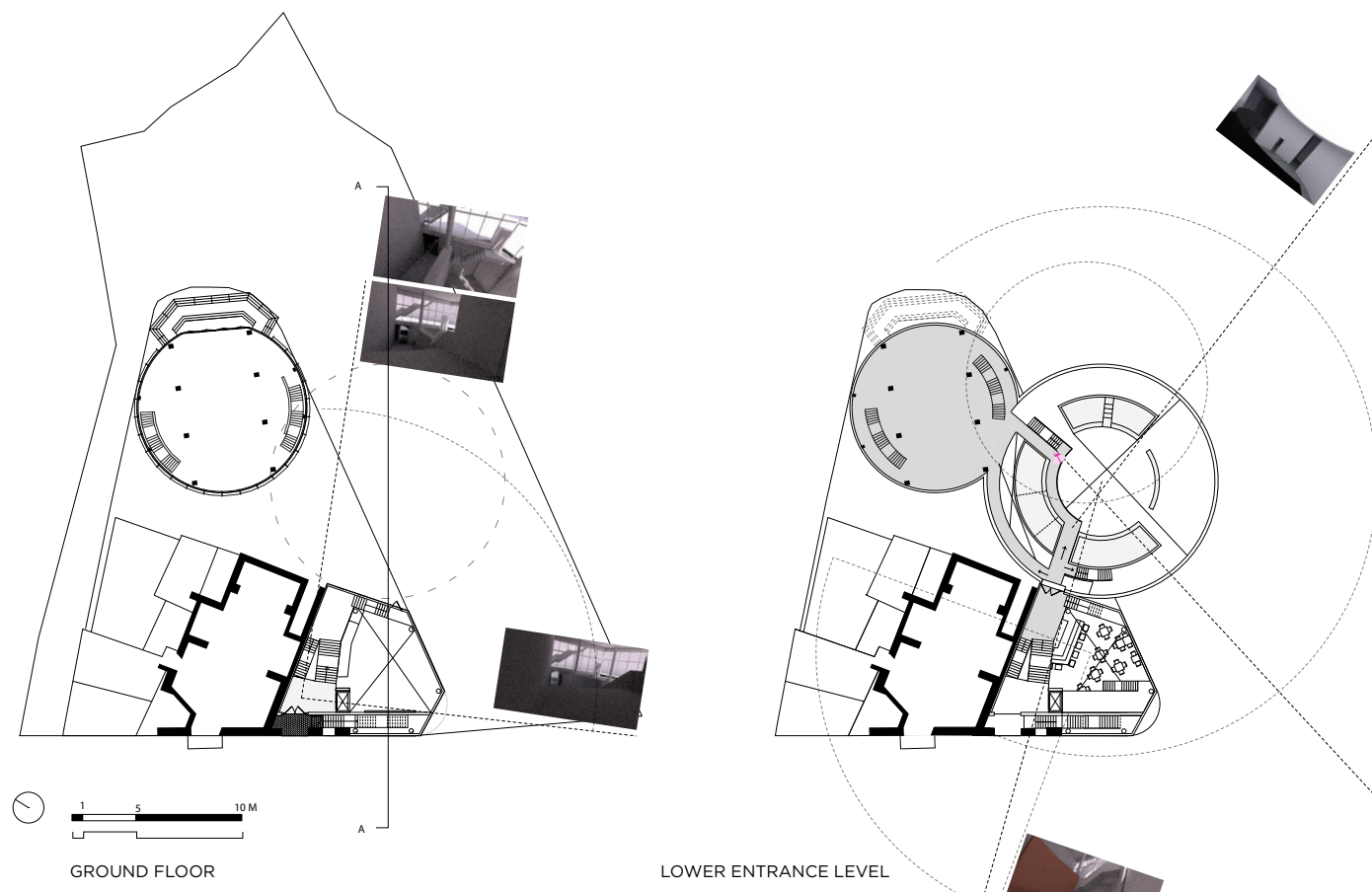

LOWER ENTRANCE LEVEL
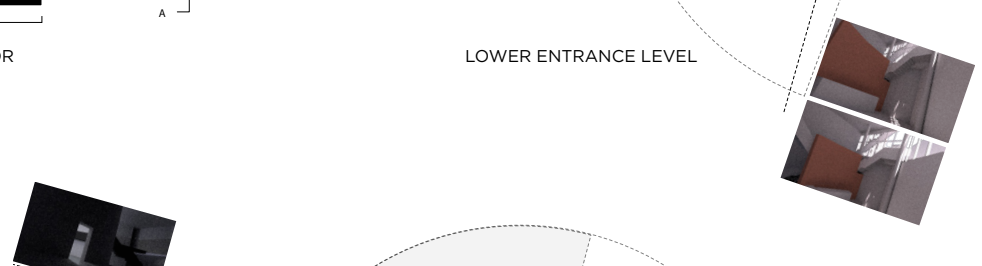

Figure 9.12 Ground level, Second

level and basement floorplans

showing selected FPR views. 


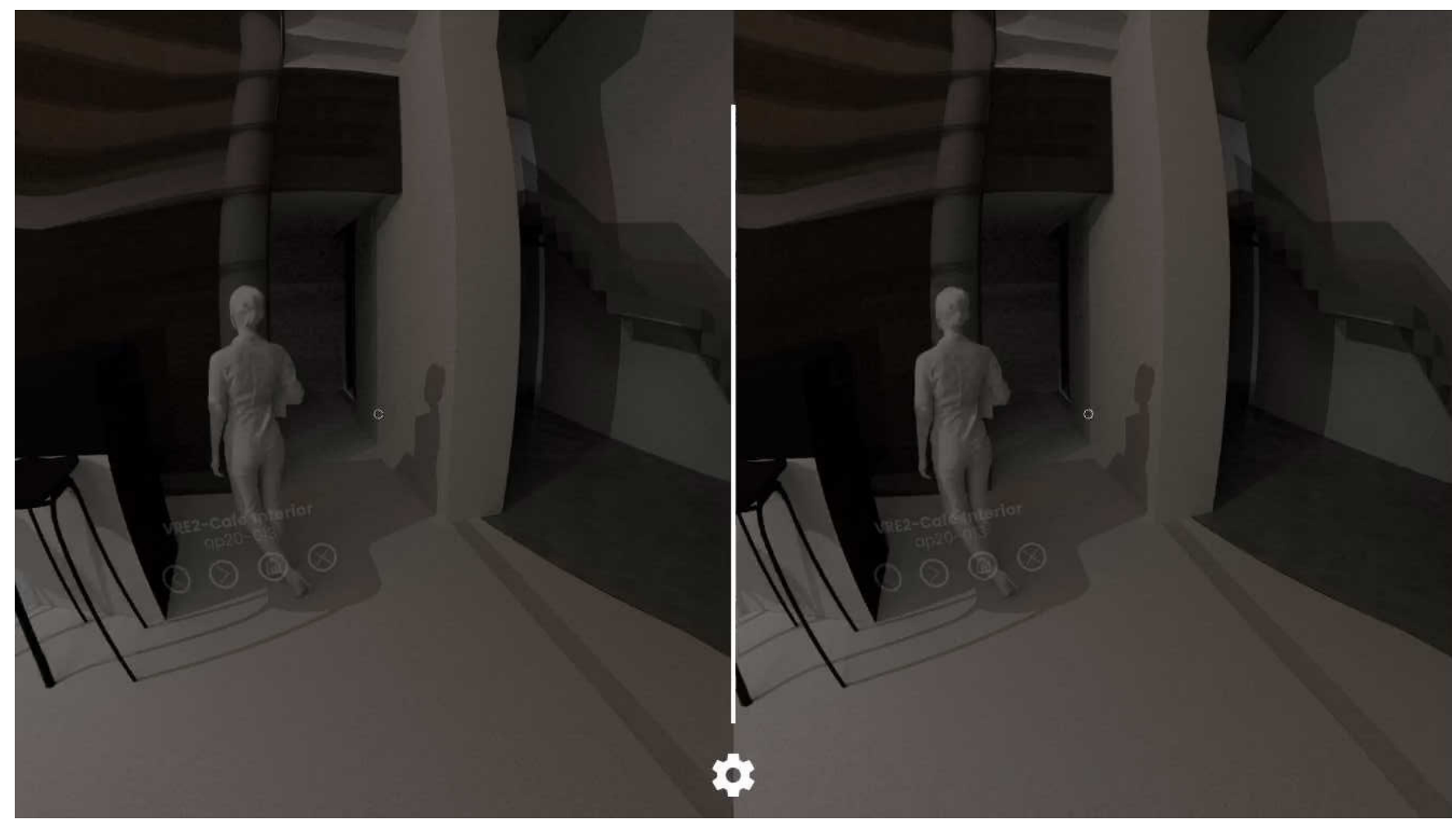

Figure 9.13 Video Capture 2 [ Café

interior]

Soundtrack: Café ambient sound

The overlaying of ambient café sound

heightens the experience of immersion

as the perceiver engages their vision and

their hearing. 


\section{ENDNOTES}

1 Edward T. Hall, "Proxemics and design" vol. (winter 1971), p. 24-25, 28, quoted by Lang, p. 85

2 Pallasmaa quotes Berenson, Pallasmaa 48

3 Pallasmaa 52 
"Every work of arch may be considered as an organic whole made up of primary, secondary and contingent parts which stand in a specific volumetric relationship to one another. Where organic structures are concerned, this relationship is determined by nature itself in such a way as to meet the particular fuctional needs of individual structures. In respect of architectural structures is it determined by man...The proportional relationships between various parts, and between each individual part and whole, is determined partly by considerations of stability, party by the need for comfort and partly by the architect;s desire to create a particular impression.

-Alois Hirt. "Why holism is only part of the game," The Theory of Architecture: The definition of architecture. p. 103 


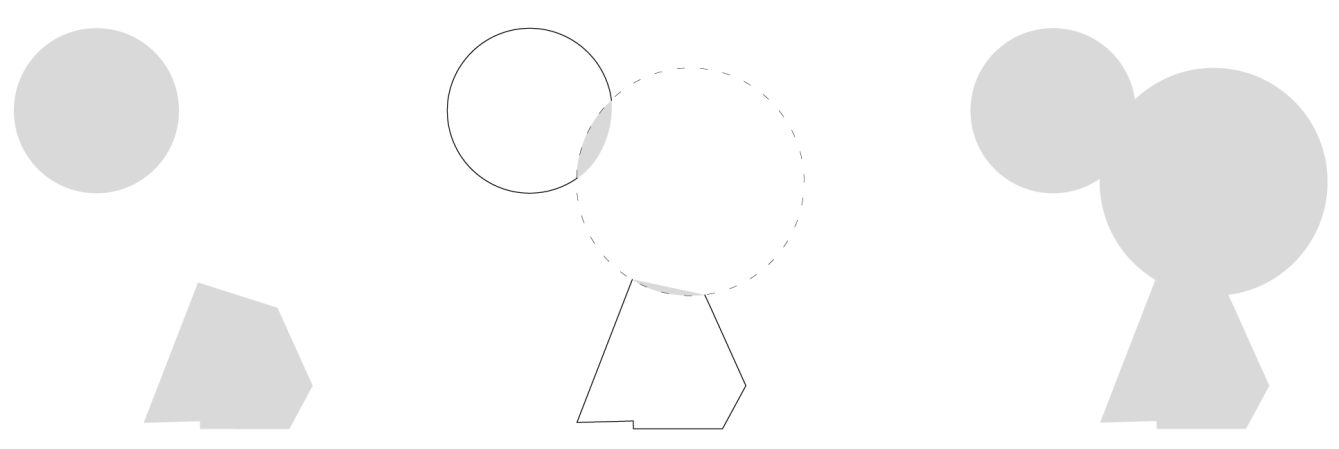

\title{
09 Building Scale: Information for perception
}

Figure 10.1 Diagrammatic plan of cafe, gallery and chapel as they overlap underground

\author{
Scale of Fitness/Building scale \\ Subterranean Exhibition Gallery
}

Building scale can be defined by designing with an awareness of scale. Similar to immersive scale, the quality of scale at the level of the building is provided by observing and defining how elements of the building fit together in space within a virtual environment. Building on the ability provided by immersive scale to consider the 360-degree of a space, with building scale parts can be evaluated as 'integrated wholes' and not unconnected elements. Building scale creates a sense of place by endowing space with value in the awareness of scale of the building, its components and how they fit together. Most importantly, this scale provides the information for perception. The qualitative approach evaluates the scale of geometry, texture, materiality, as well as certain amounts of colour. If we were to remove all the relational elements that provide scale that allow us to compare them and project our impressions of scale, what remains?

Once we test building scale for 'fitness' in its relationship to the whole (as a gestalt), we can begin to evalute and iterate its size by our projected reading. 
The basis of scale is founded on the human body arises from our awareness of our own physical size and the relation of everything else to that size... When we perceive/understand the scale of an object, by extension we understand the scale of the room. Designing for scale includes the consideration of hierarchy or sequence, rhythm/repetition, precedence/ emphasis, tension, and time. (Orr 22)

Hierarchy of architectural values; augmented by variables such as shape, detail, and treatment of surfaces and edges" 1 The focus is not 'successful design', but a recognition and dealing of scale. Materiality, texture, form, and building components reveal scale to us. Elements such as stairs, furniture and people aid in the projection and comparison of scale and how two materials meet.

Natural materials express their age, story of their origin, history of human use...allow our vision to penetrate their surfaces. (Pallasmaa 34)

As an intellectual rebirth of the ideals and ideas of classical culture, the Renaissance marked a dramatic change in a 'crudely cannibalistic attitude toward the past'. ${ }^{2}$ Instead, the ideas and forms of ancient architecture were drawn precisely and studied, leading the way to the ideas of High Renaissance. One such architect who came to Rome to study antiquity was Donato Bramante. Bramante's masterpieces stand out for their creative fidelity to the past. Bramante expressed form and meaning through the historical relationships to the city and history of Rome. It is worthy to examine his Tempietto to begin to understand the meanings behind his sources.

\section{The Circular Plan}

Tempietto circular for several reasons, 'the round plan embodied the perfection of Platonic geometry. It recalled the Pantheon, which Palladio would later describe as the "image of the world". 3
The circular colonnade suggested a likeness to what was thought to be the Temple of Hercules near the ancient Forum Boarium.

The tempietto's diameter is the same size as the Pantheon open oculus. His design choice was made by an "[interest] in how these apparent opposites could be reconciled"4 By creating two contrasting natures, where one is figure and the other is ground, Bramante created and built on larger pieces of a great conceptual puzzle of Rome.

In an effort to include this humanist view into the design within the VR model, the chapel design integrates two cylindrical volumes. The use of the circular plan recalls the form of Bramante's Tempietto. It reflects the perspectival reflection

Figure 10.2 San Bramante's Tempietto mimics the diameter of the Pantheon oculus creating a figure-ground relationship

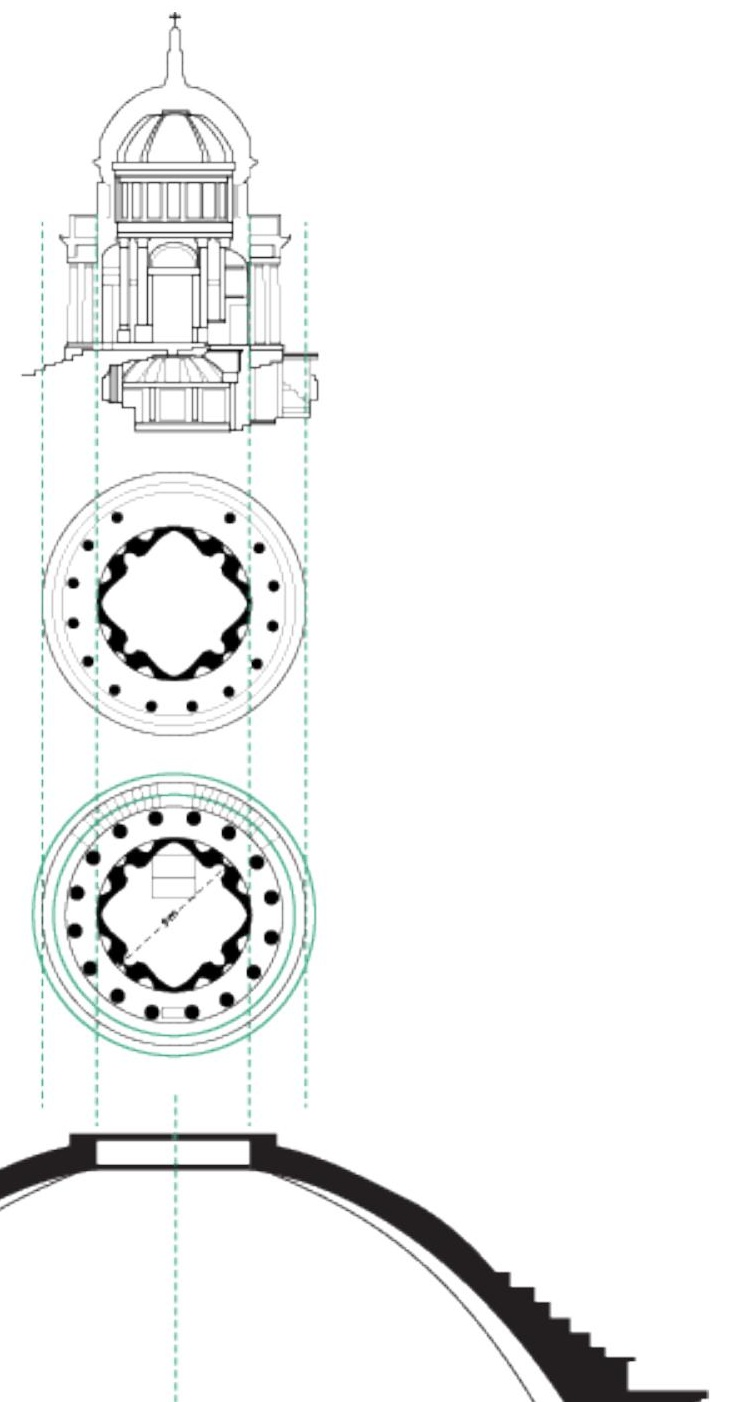


Bramante saw in the Pantheon oculus and the Pantheon itself, as well as it's claim as the 'perfect' shape that is inclusive of many cultures and religions.

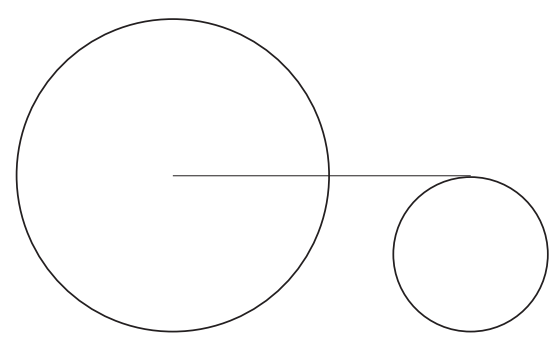

Figure 10.3 The new Pathway (underground tunnel) diameter is $21.5 \mathrm{~m}$, half the diameter of the Pantheon (43m).

The cylindrical volume is suggestive of the 360 panorama. Within VR, immersion allows the user to conjecture a sense of space more than a perspective model. By engaging in a cylindrical condition of 360 degrees; the whole-image medium can be explored from various perspectives. The third design intention examines the awareness of scale of the building, its components and how they fit together, having the designer:

[Design Intension 3] To consider the 360-degree of the visual experience of place beyond what fits into a render frame

Perspective is a crucial part of performance. VR helps us better understand and visualize our designs so we can craft spaces defined by well-informed aesthetic and functional design decisions by regularly checking back with the form and qualitative, participatory approach to make any necessary adjustments.

The 'laws of perspective' are implemented in the design by the framed views at the terrace and the orientation of the nave in the direction of the bridge. 


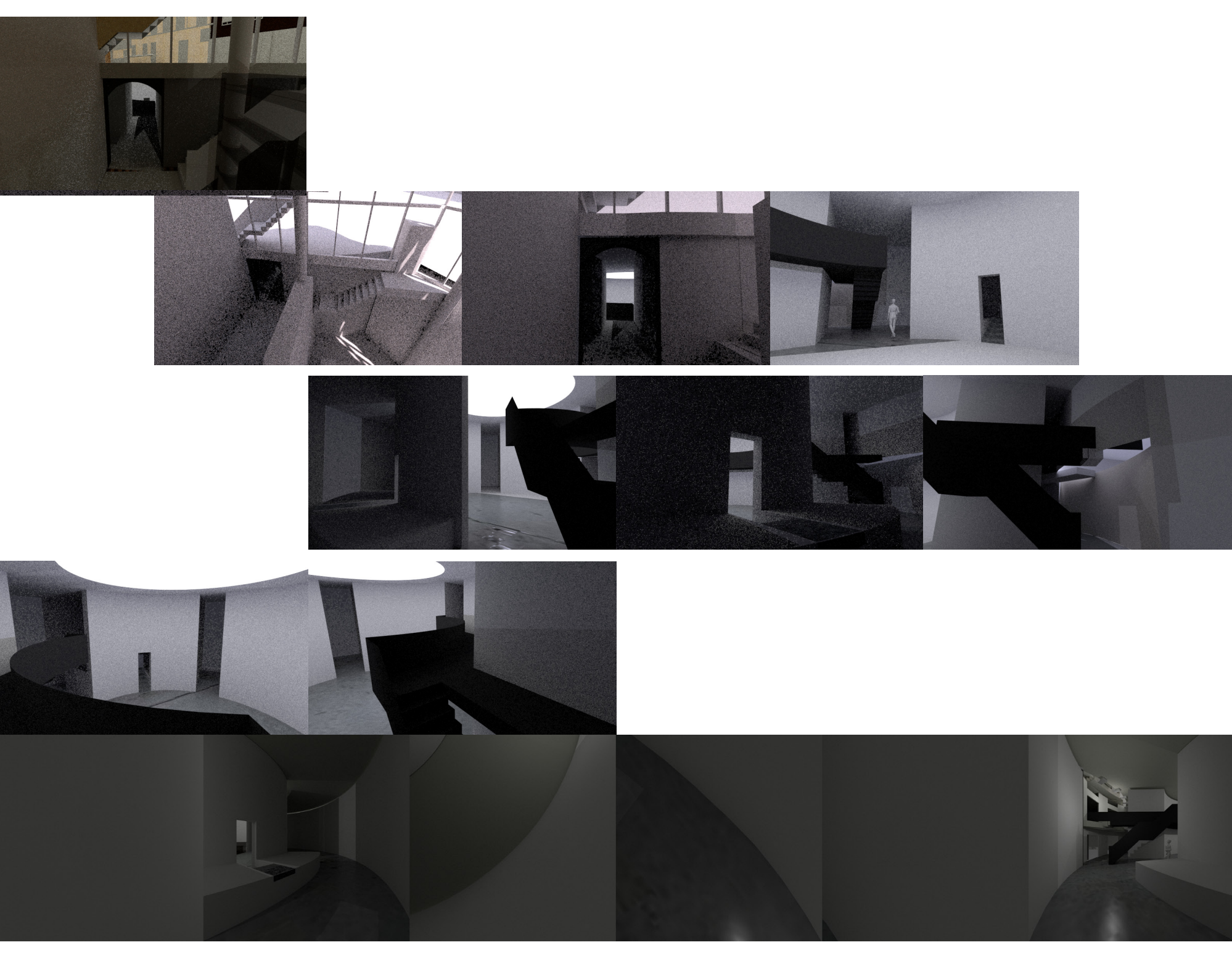


Allows the navigator to design with an awareness of scale of

the building and its components, adding an experiential sense

of space in how the building fits together in relationship to the

navigator. Exposure to experience adds an understanding of

relationships by extensions of scale and how scalar qualities

interact and reinforce one another.

For the designer, perceiving the spatial qualities of

the space with VREs allows assement, followed by iteration or

redesign.

Building design 'that handles scale well' will be

perceived as a gestalt ('a structure whose totality is greater than

the sum of its parts-because the functions of scale operate in

simultaneously supportive ways' (Orr, 11). Bad scale is "unfitness"

of various component parts.

In VR, we can examine appropriately

scaled design. This includes:

proximity, privacy, and territoriality;

to be addressed in immersive scale.

VR allows us to conjecture the sense

of scale in a space to accomplish an

(end) experience

Figure 10.4-10.12 [LEFT TO RIGHT]

A processional sequence of entering the gallery showcasing THRESHOLDS, OPENINGS, DOORWAYS. Fragmented

ENDNOTES

images do not relay the immersivity

when viewed through a headset. Figure

$1 \quad$ Orr 23

10.13 FPR of the threshold between the

2 Mayernik 48

gallery and the lower chapel level.

ibid 49. 


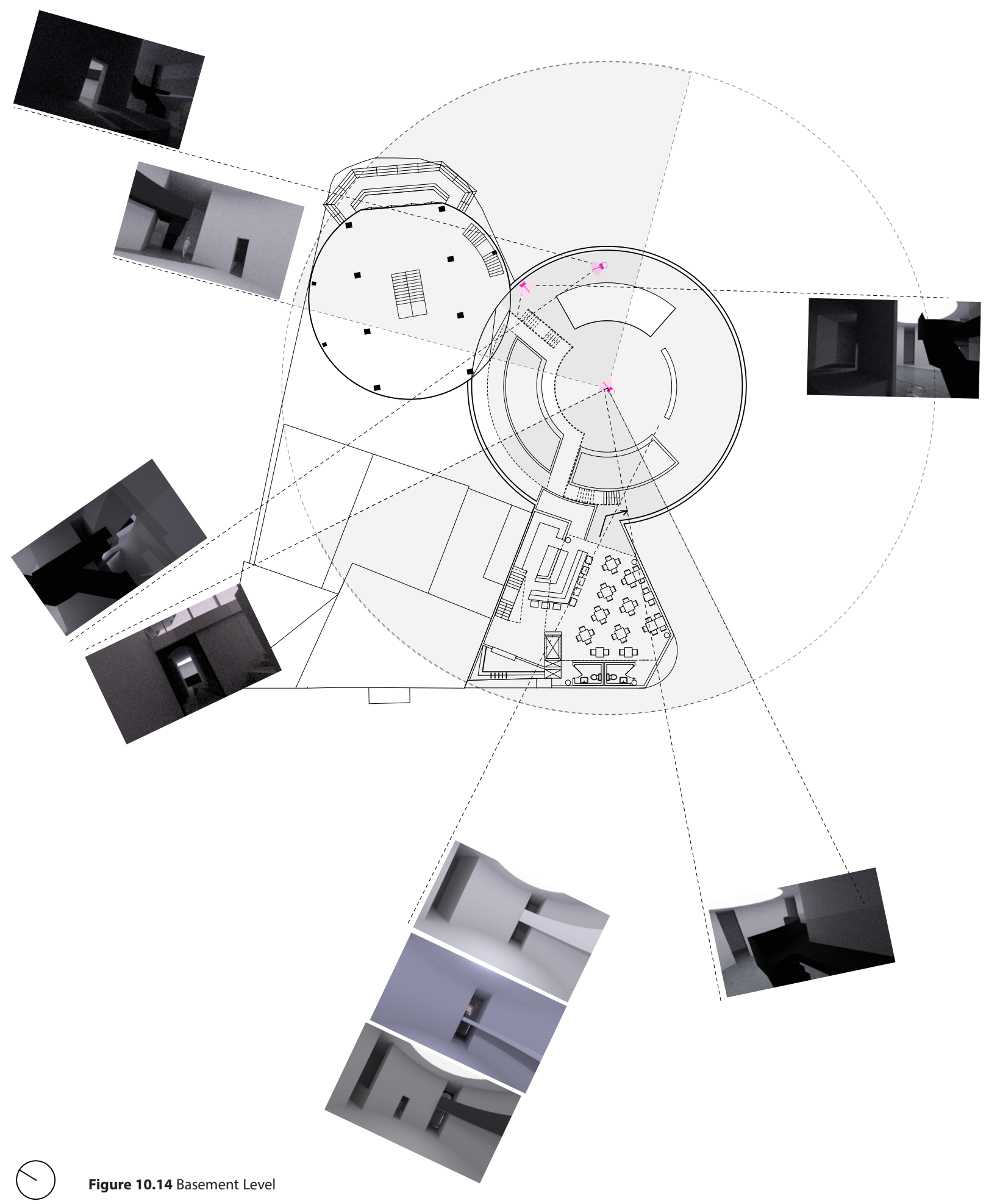


Figure 10.15-10.20 [LEFT TO

RIGHT] Series of threshold

openings to challenge the

perception of scale.
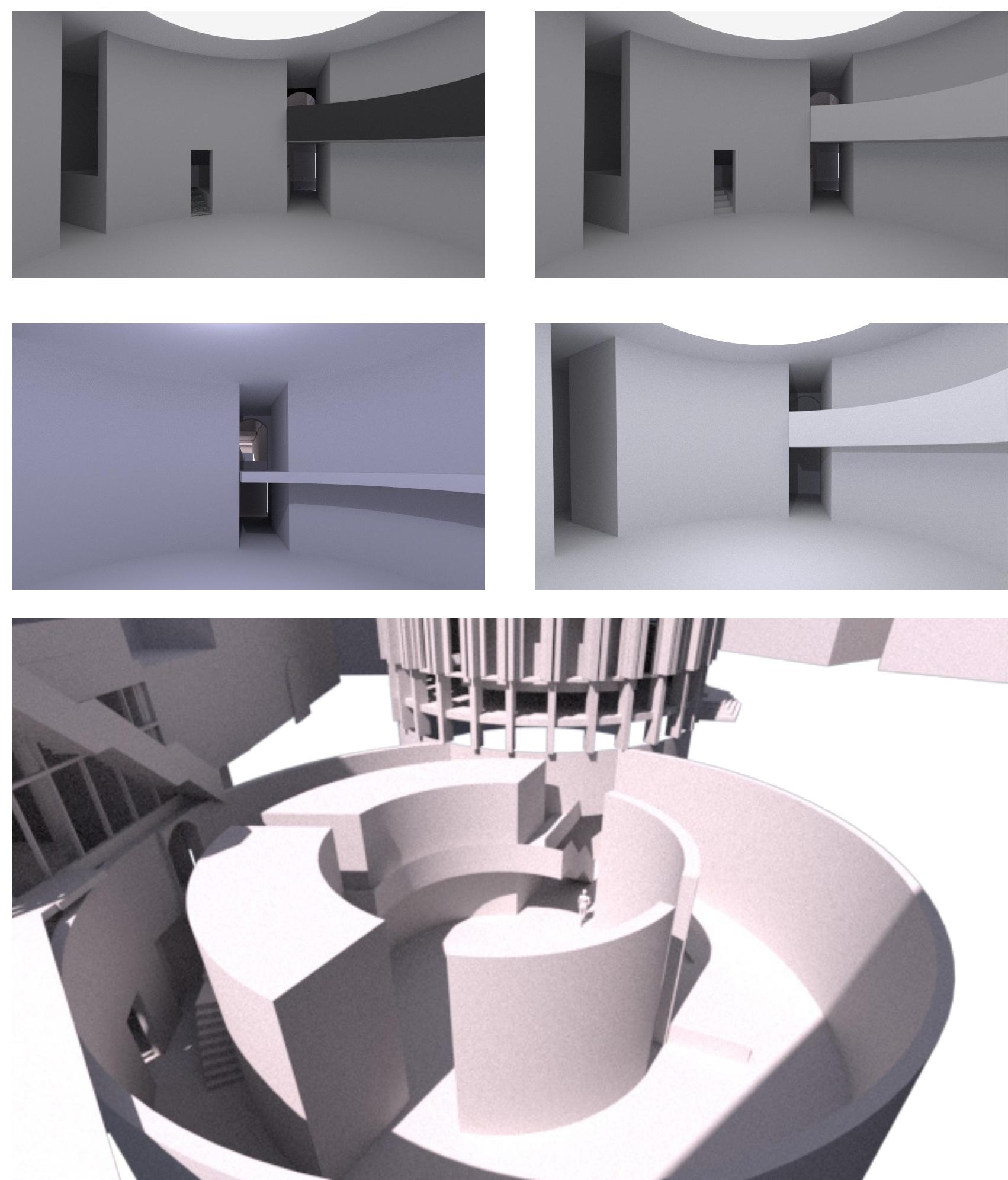


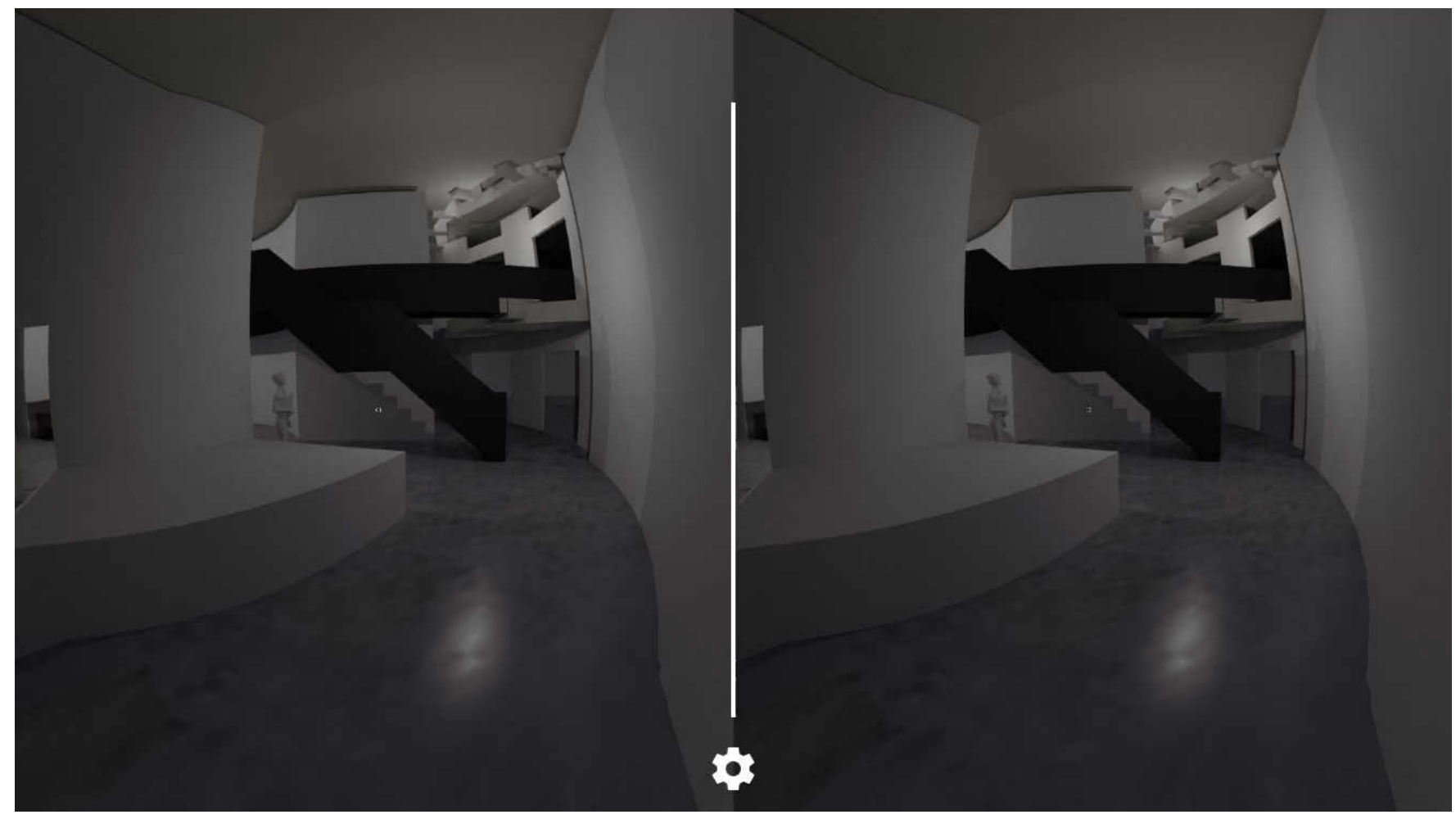

Figure 10.21 Video Capture 3 [Subterranean Exhibition Gallery and Threshold] Evaluating building scale through geometry, texture, materiality and colour 


\section{ENDNOTES}

1 Picard, Max. The World of Silence. Eighth Day Press, 2002.

2 Jongerius, Hella, Breathing colour, exhibition, 2017.

3 Mayernik 88

4 Bachelard, Gaston, and M. Jolas. The Poetics of Space. Penguin Books, 2014.

5 ibid

6 Gibson in Allen and Otto. Jonassen,

David H. Handbook of Research for Educational Communications and Technology: a Project of the Association for Educational Communications and Technology, Macmillan Library Reference USA, 1996. p212. 
Just as ivy grows round a wall for centuries, so the cathedrals have grown around the silence. They are built around the silence. -Max Picard, The World of Silence ${ }^{1}$

It's unbelievable how colours can breathe if you use them right. -Dutch industrial designer, Hella Jongerius ${ }^{2}$ 


\section{Relational Scale: Activity of perception/ Visual Awareness}

\section{Scale of Fitness/Building scale Subterranean Exhibition Gallery}

Gaston Bachelard claims, "our comprehension of space, however confined or expansive, still affords an opportunity to encounter the boundaries of the self just as they are about to give way." $\mathrm{He}$ further notes, "inhabited space transcends geometrical space". To an observer it is the first impression, for the designer it is the overall impression, where both are caused by the immediacy of the experience. The immediacy is coupled to our awareness. Gibson states,

The act of perceiving is one of becoming aware of the environment or picking up of information about the environment...nothing like a representation of the environment exists in the brain or the mind which could be in
The real work of making Rome beautiful was driven by the desire to make her speak. It was only because there was some degree of consensus about what she would say that we have inherited the poignantly beautiful city that she became overtime. -Mayernik ${ }^{3}$

greater or lesser correspondence with it-no 'phenomenal' world which reflects or parallels the 'physical' world. ${ }^{6}$ While the design is rooted in the physical world, it does not recreate it. A stereoscopic image provides a very close impression to the atmospheric qualities a space creates. Pallasmaa describes atmospheric perceptions as:

Atmospheric characteristics of spaces, places and settings are grasped before any conscious observation of detail is made... Neurological investigations suggest that our processes of perception and cognition advance from the instantaneous grasp of entities towards the identification of details, rather than the other way around. (15) 
The chapel interior acts as the major space to challenge relational scale fufilling the fourth design intention.

\section{[Design Intension 4] To create a narrative experience that} questions where we 'fit' at various scales, in attempt to challenge human scale and perception

When we engage in the activity of perception, we relate our perception to our own propreoceptive understanding of our body's dimensions.

\section{i. Challenge human and temple scale.}

It is interesting to note that scale becomes intimate at the perimeter, when in close contact with something [See Figure 8.5]. We are challeged by the size or height of spaces when we can no longer relate to it.
The window sets up the "laws of perspective" by framing the landscape into what Glbson defines as aperature vision. At the same time, there is an ambiguity of scale in intimately framed views. Casanova+Hernandez architects craft an intimate sensory experience with perception by framing views in a self-made park in Switzerland [Figure 8.1-8.4]. Another example of this can be seen in Rome, through the Aventine keyhole where the spiritual, mysterious and suspenseful event can be experienced. Pallasmaa cautions that, "During overpowering emotional experiences; we tend to close off the distancing of vision". ${ }^{1}$ He claims, "Deep shadows and darkness are essential, because they dim the sharpness of vision, make depth and distance ambiguous, and invite unconscious peripheral vision and tactile fantasy."2 There is an opportunity to build on the poetic experiences of a spiritual space by challenging scale and expectation, such as the ability to play with volumes and heights of sequential spaces. Framing view can heghten experience and curiosity, but it cannot fufill our desires for whole body experiences.

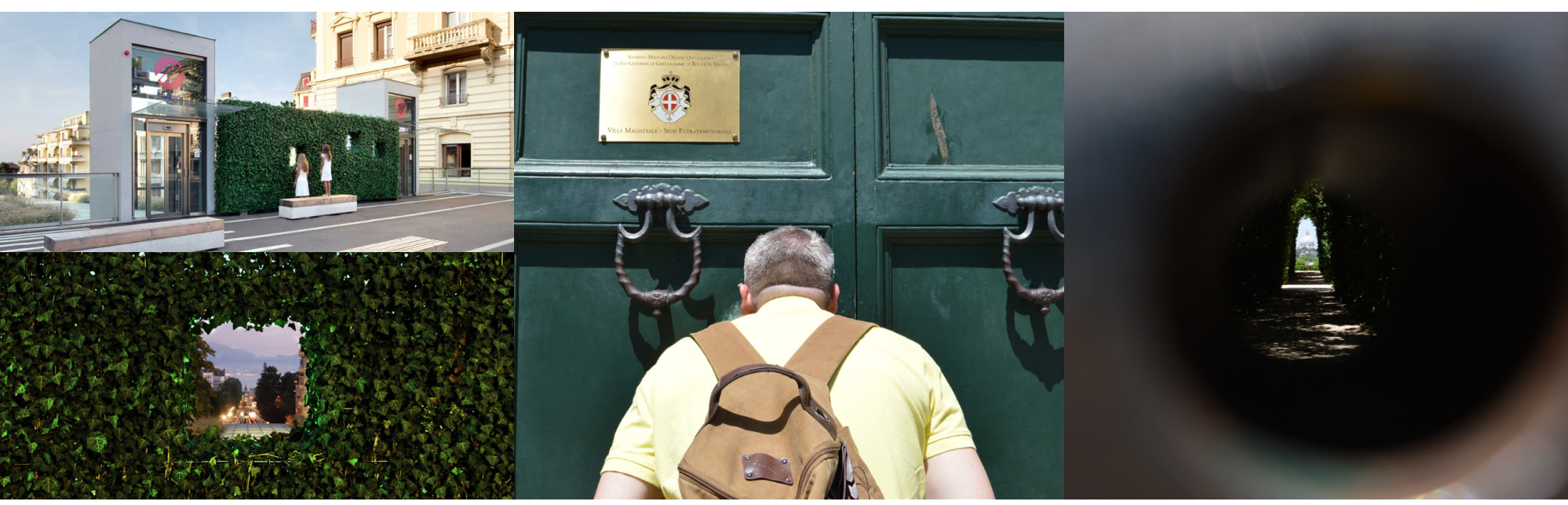

Figure 11.1-11.2 [LEFT] Public Space, Lausanne Jardins 2009 by Casanova+Hernandez architects. Lausanne, Switzerland (2008-9) Figure 11.3-

11.4 [RIGHT] The Aventine Keyhole, framing St. Peter's right in the center at the Santa Maria del Priorato (18th century) by Giovanni Battista Piranesi 


\section{ii. Reawaken our sensibility and sensitivity to spatial} experiences through materiality

Materiality has the capacity to create sensory experiential quality in architecture. Simulation in VR can allow for exploration of material for various sensory experiences. Engaging in the site specificity, in this case, a Roman context, local identity can be examined within the scope of materiality. This relates back to building scale. As an exploration of material simulation requires a consideration of material, structure and finishes, to be developed in parallel with the design. Light becomes a material when we design with materiality from conception iii. Awareness of natural/artificial light sources; sensory qualities

Light is a unifying element in the order of spirituality and worship. The divine light or white light is what is said about the ideas of the after life or the ideas that hint of something greater than us. Different use of light represents space differently. Our navigation within the space engages in a certain experience. There is no form without light.

Light has turned into a mere qualitative matter; window has lost its significance as a mediator between two worlds; turned into a mere absence of a wall. (Pallasmaa 51)

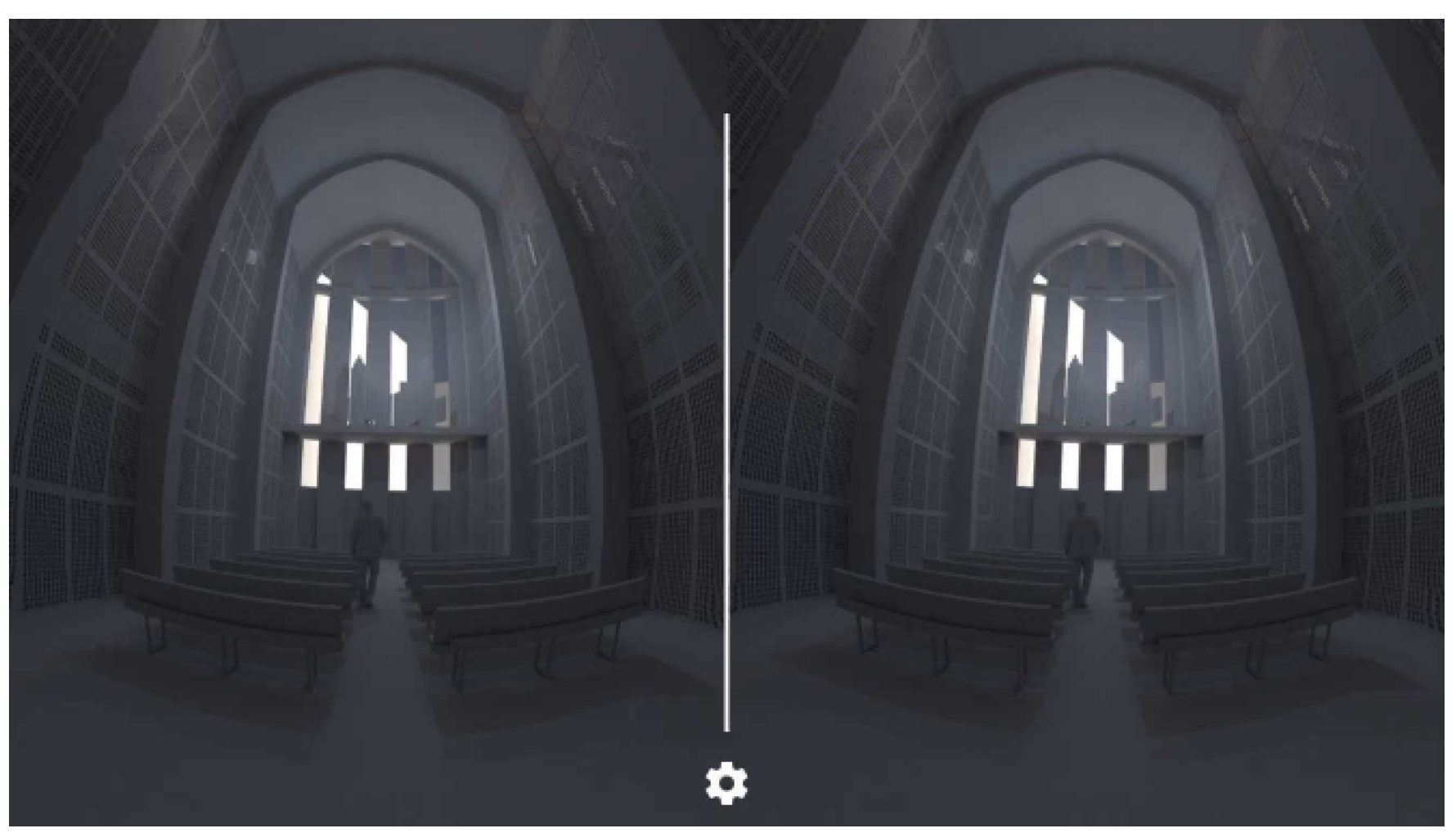

Figure 11.5 Video Capture [Chapel Interior]

Examine where we 'fit' at that scale 


\section{'Beyond measure'}

The intimately immense: Bachleard

says we do not need a large number of trees to give a sense of immensity.

Figure 11.6 [TOP LEFT TO BOTTOM

RIGHT] Chapel Interior Light studies as

FPR screenshots.

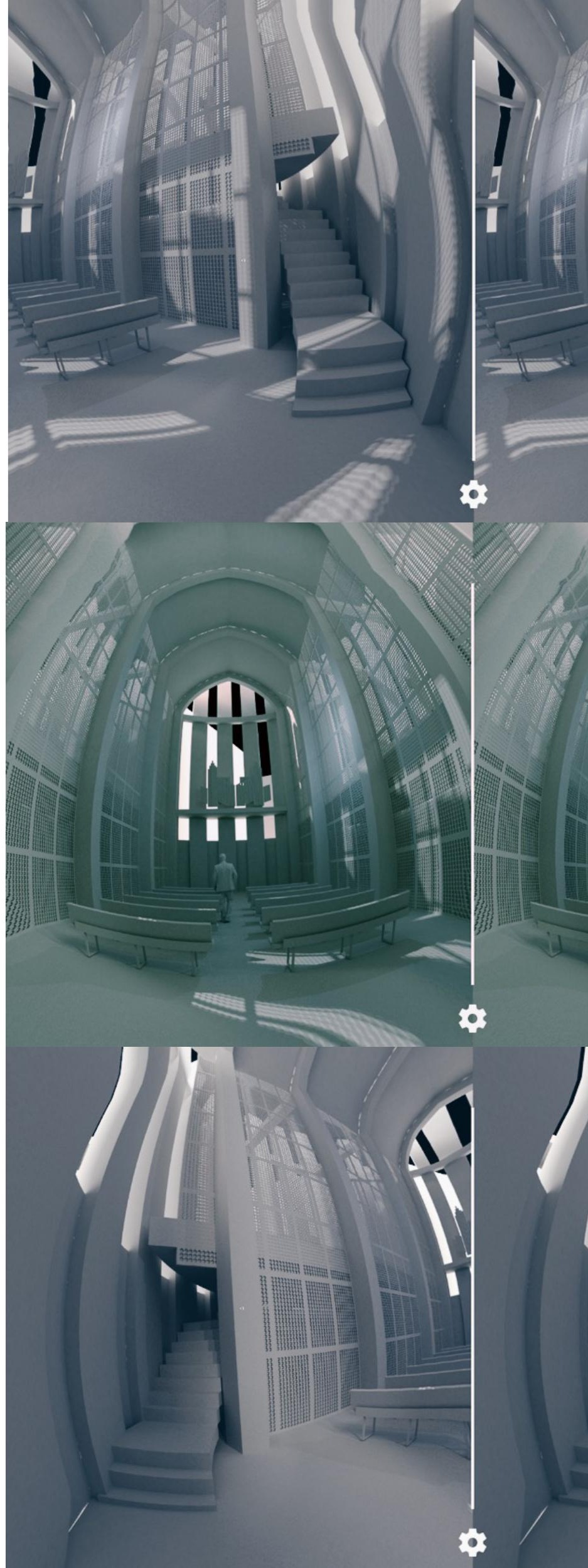




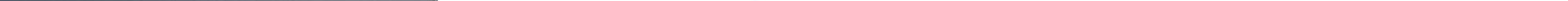




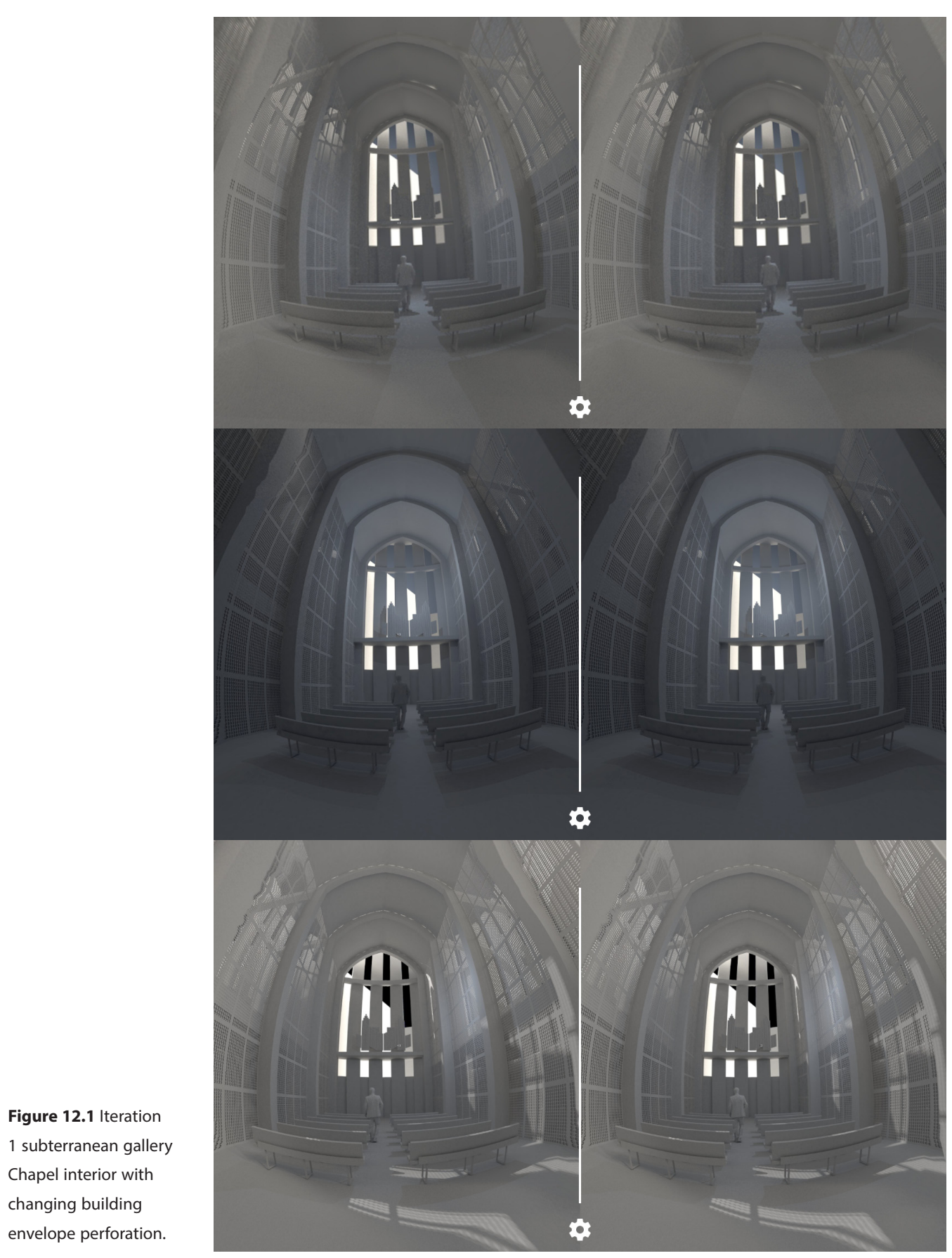




\section{Conclusion: A form of realization}

Historical ground in perspective continues to influence our relationship to the world. Temple poses the premise that architecture,

"not only provides the setting for the 'here and now' but also bears witness to the history of human situations. In each case architecture becomes witness of human events. This largely unrecognized dialog underlies our perspective outlook today and serves as an abiding reference in our understanding of the nature and meaning of architectural representation." (26)

The investigation of perspective, posited to sense and perception in experience, frames architectural representation as the mediator between concepts, practice and spatial qualities in telepresence.

Experience excites curiosity.

And curiosity drives experience.

William James notes, "In its inner nature, belief, or the sense of reality, is a sort of feeling more allied to the emotions than to anything else." And in this faith, "The true opposite of belief are doubt and inquiry, not disbelief". ${ }^{1}$ We engage in the look and feel, discovering the phenomenological qualities that one cannot fully anticipate without awareness mediated by experience. VR is the beginning of privileging the image in its ability to communicate the phenomenological and sublime qualities of spaces as it allows architecture the ability to communicate itself honestly. A poor stereoscopic rendering, lacks the sensation of immersion. A strong rendering presents digital place. There are no layers of subjectivity, and no prescribed route.

"We have come to believe that the act of perception is instantaneous with things seen and give little thought to the duration of perception. If perception, as Lyotard notes, has a Bergsonian durée horizon is the locus of this duration and the way perceptual time is stretched."

The direction of architectural representation is moving towards using the body (or rather head-on-the-body to proprioceptive movement) to access the dimensional and ergonomic relationships of spaces and elements. The ability for greater precision and insight gained from prototypes prior to their realization is the goal and the direction of development in virtual prototyping. Thus, the result is not a reflection of design intentions, but the product of something discovered in the telepresence of the virtual prototype. Often these sensations are generated within us, but must be triggered through the senses. 


\section{ENDNOTES}

1 James, William. "Chapter XXL: The Preception of Reality." The Principles of Psychology. Vol. 2, H. Holt, 1890.

2 "Program as Horizon - Frank Weiner, Meditations on Two Competition Projects, 2012." E2A Architects Zurich, www.e2a.ch/news/programhorizon\#/page1/. 
APPENDIX A 

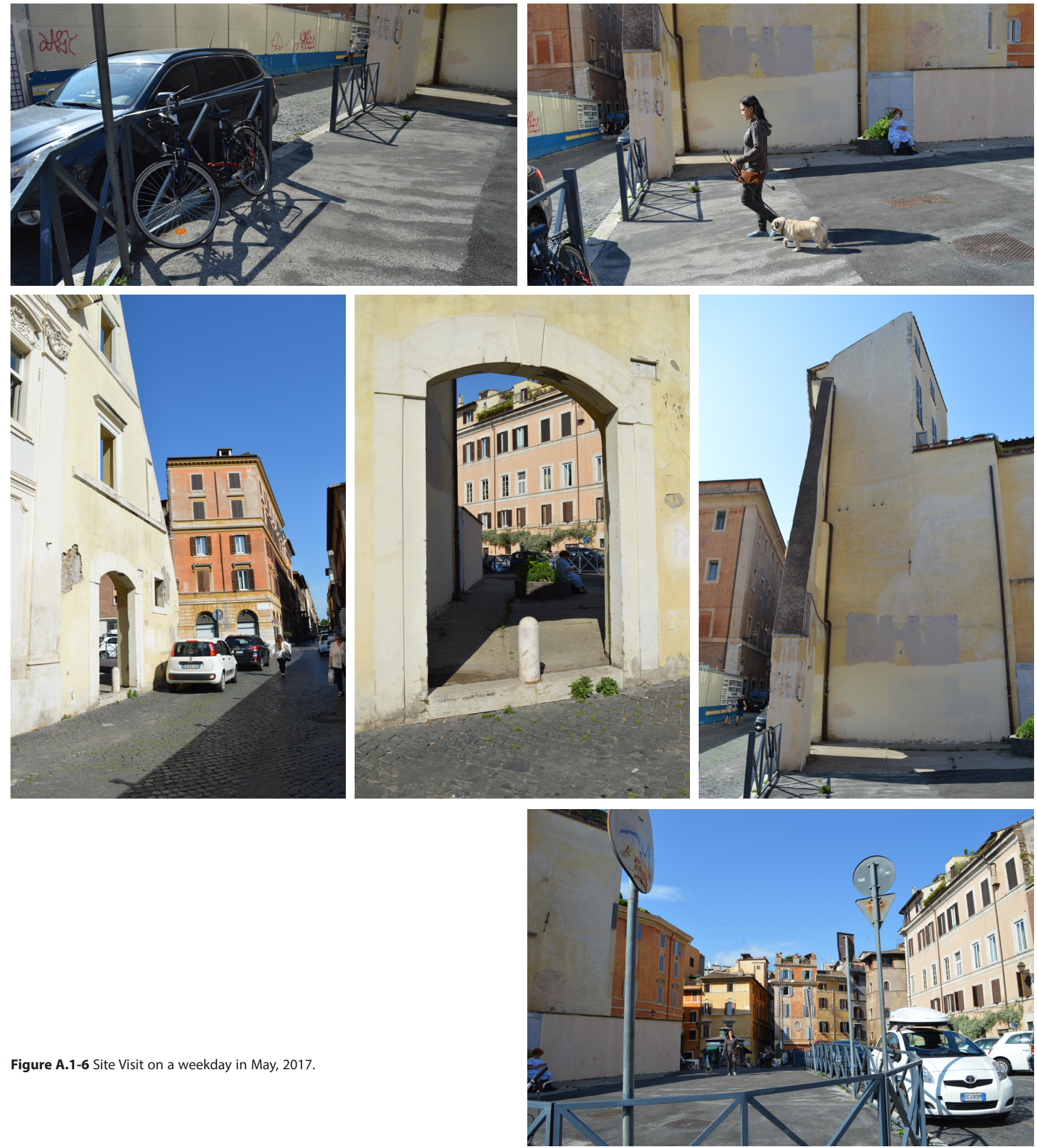

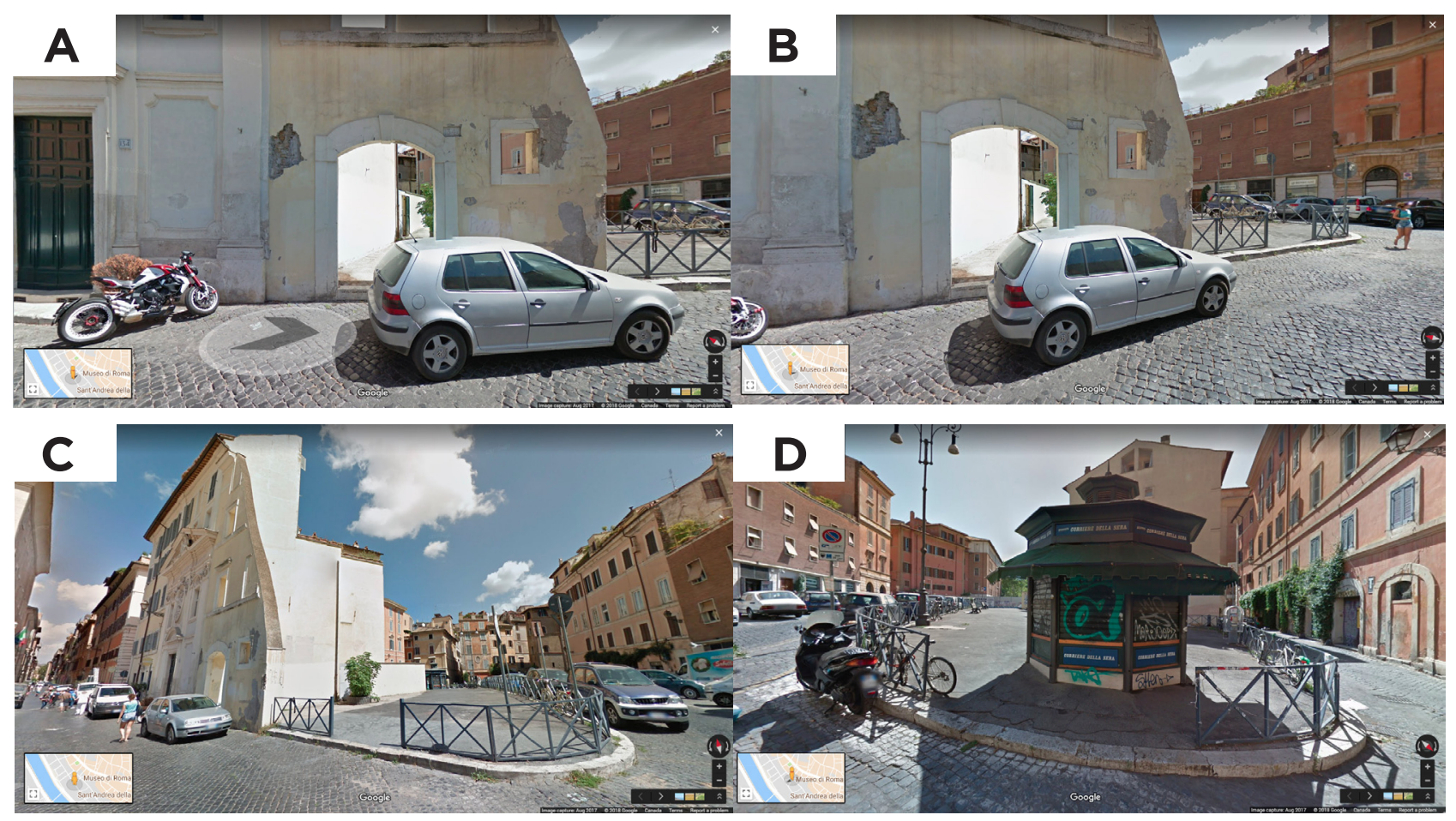

Figure A.7-10 Google Street View of site

seasonal and temporal data compared to site

visit experience and photos. Scale is often

manipulated in various views, however quotidian

lifestyle is evident. 

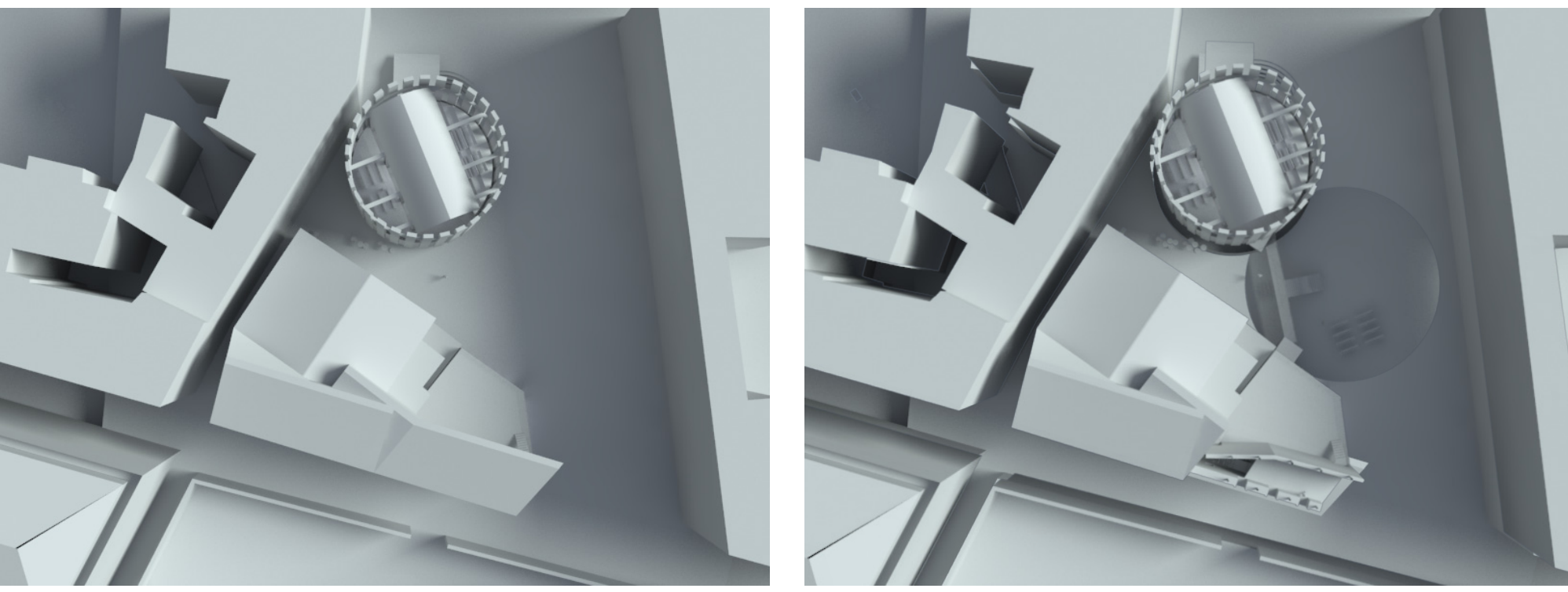

Figure A.11 [Iteration 1] Site Plan (Chapel roof removed)

Figure A.12 [Iteration 1] Site Plan (Chapel roof removed/subterrean gallery exposed)

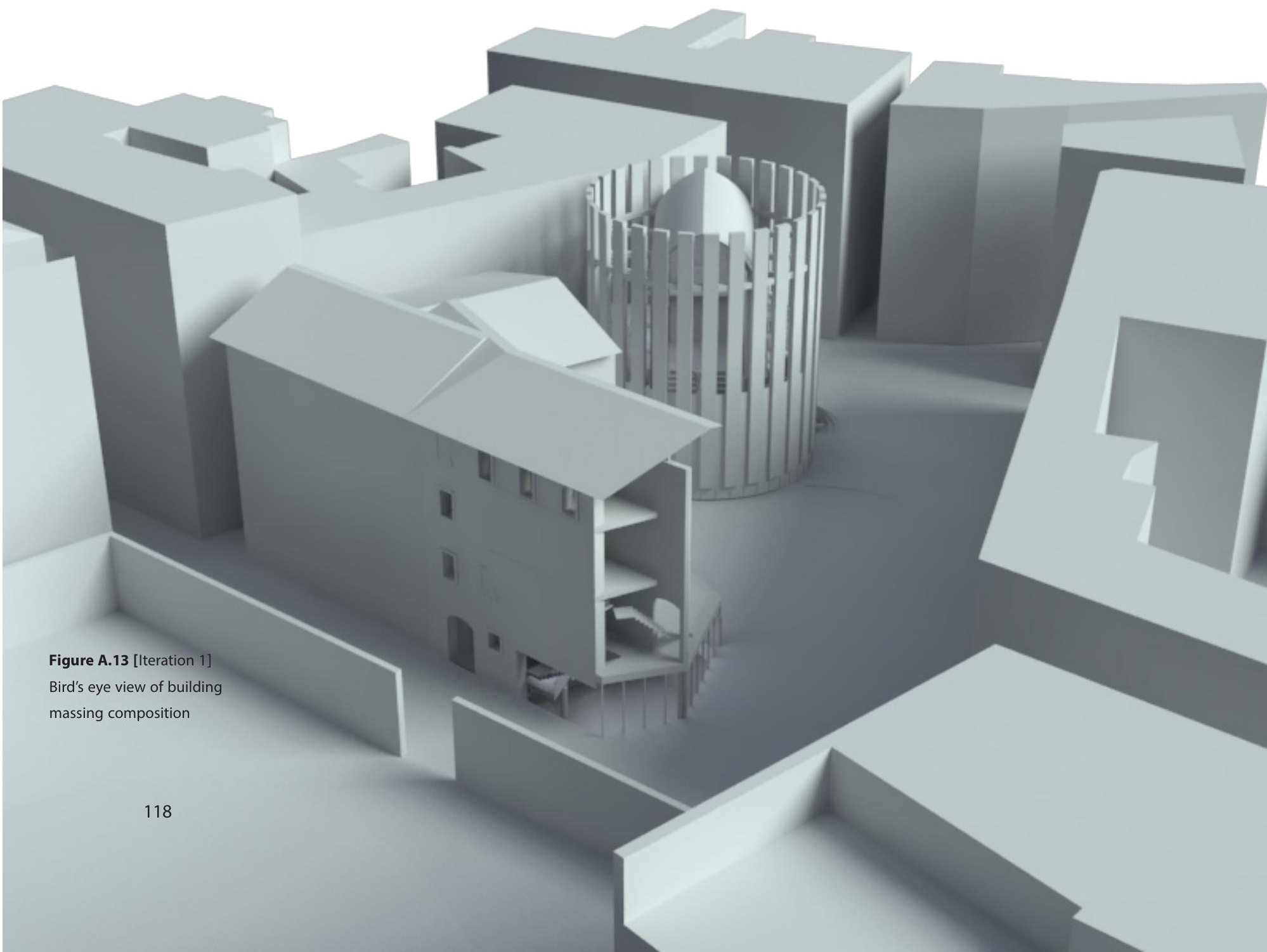




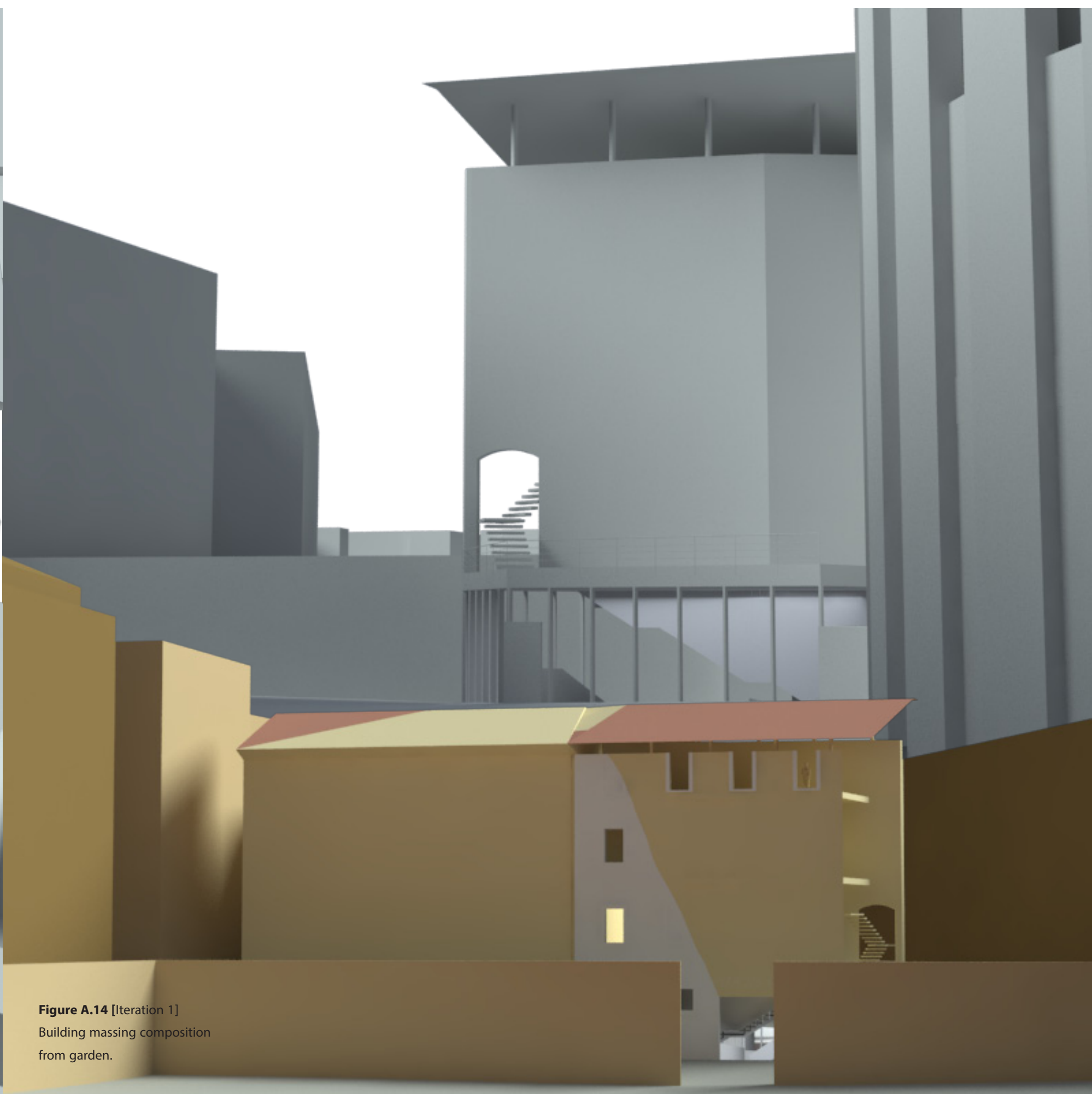




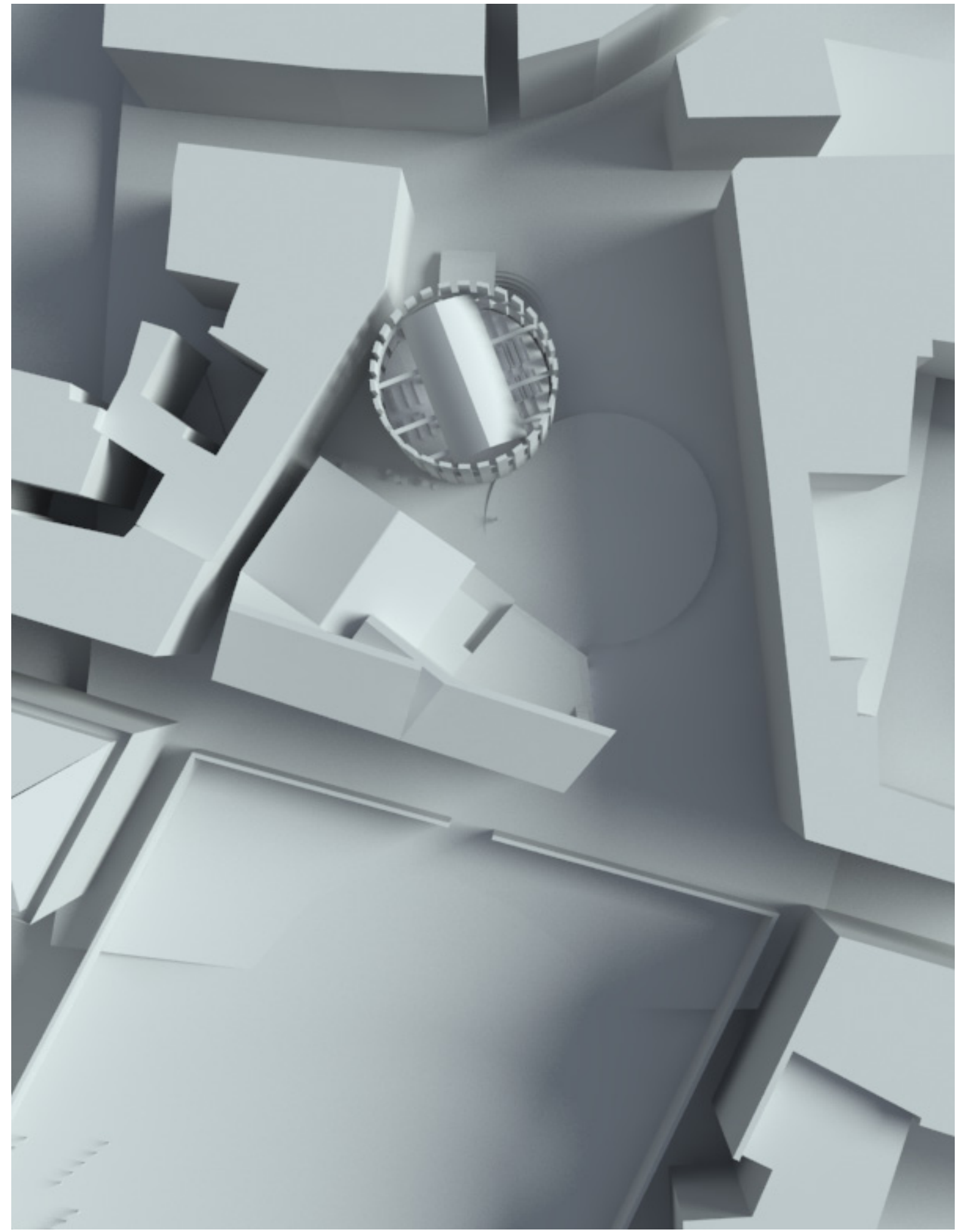

Figure A.15 Iteration I, Aerial Rendering. 
Figure A.17 Iteration I, Massing.

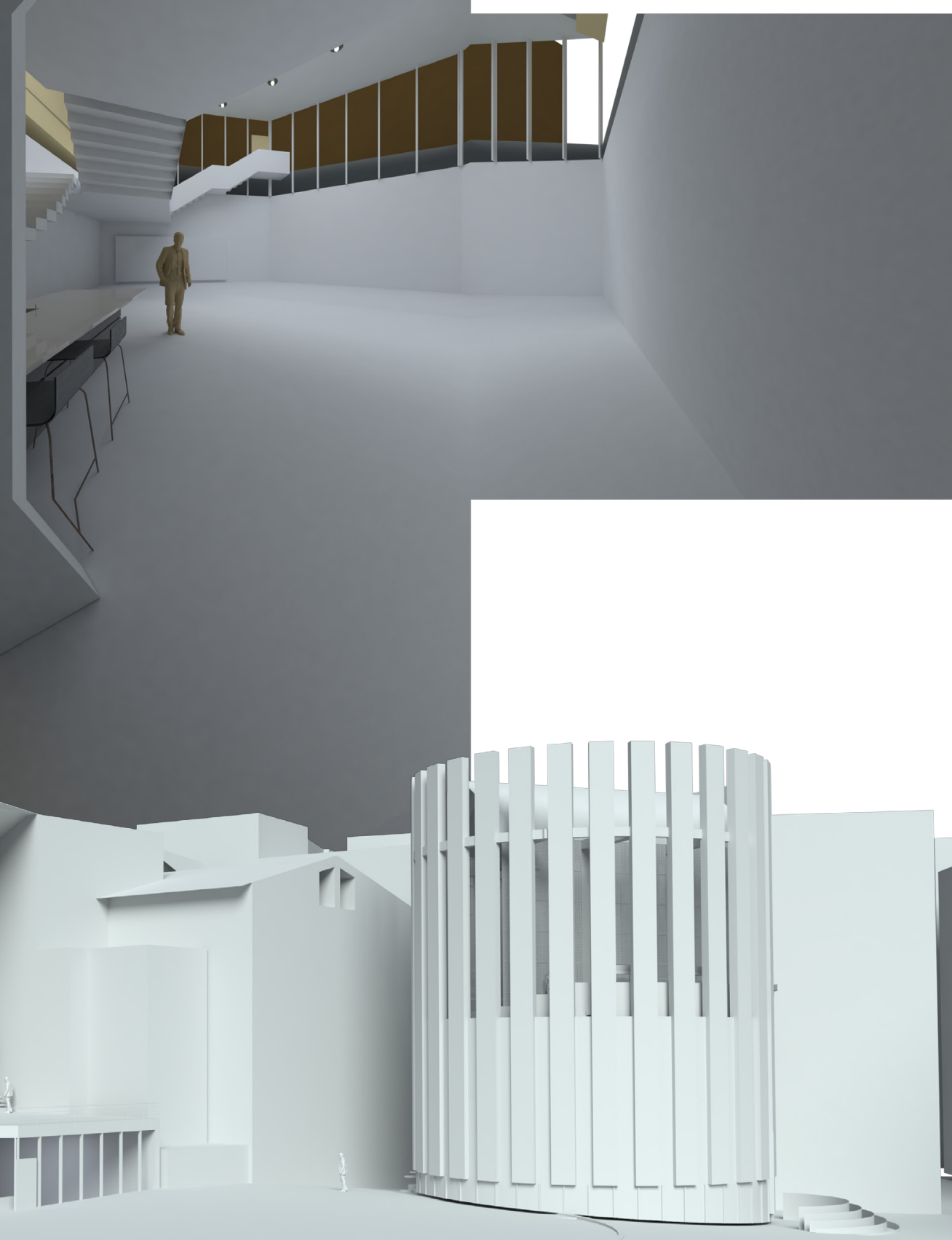




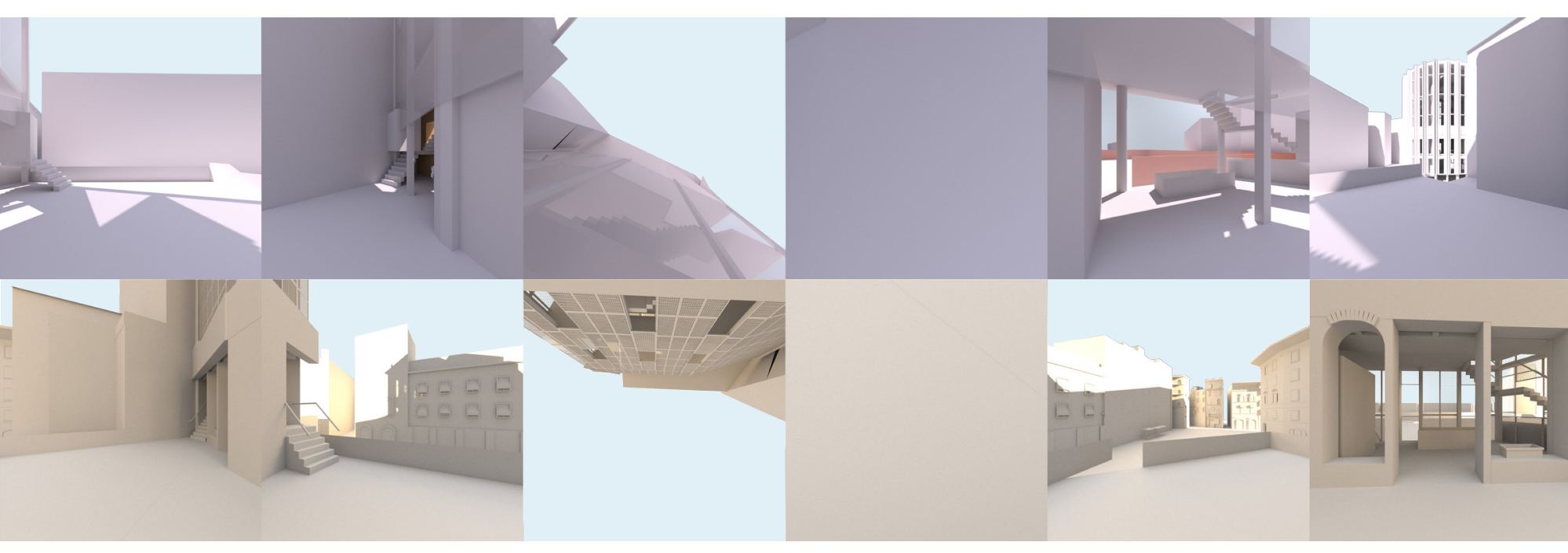

Figure A.18-21 FPR renderings. 


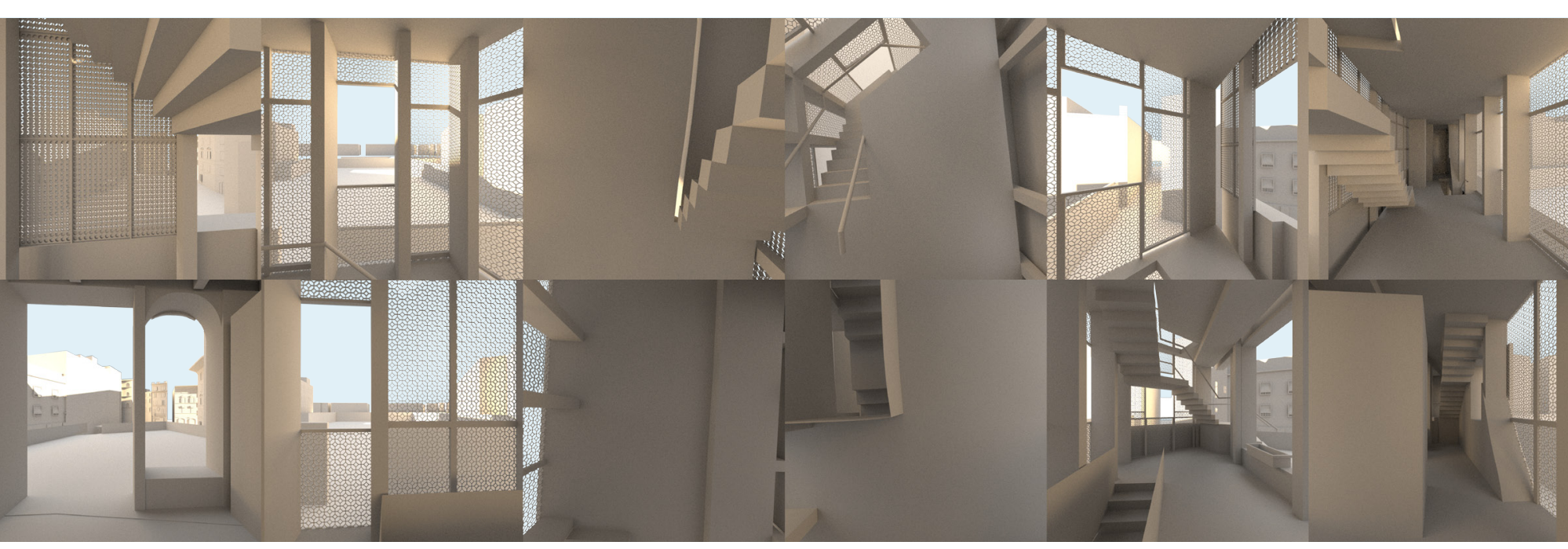




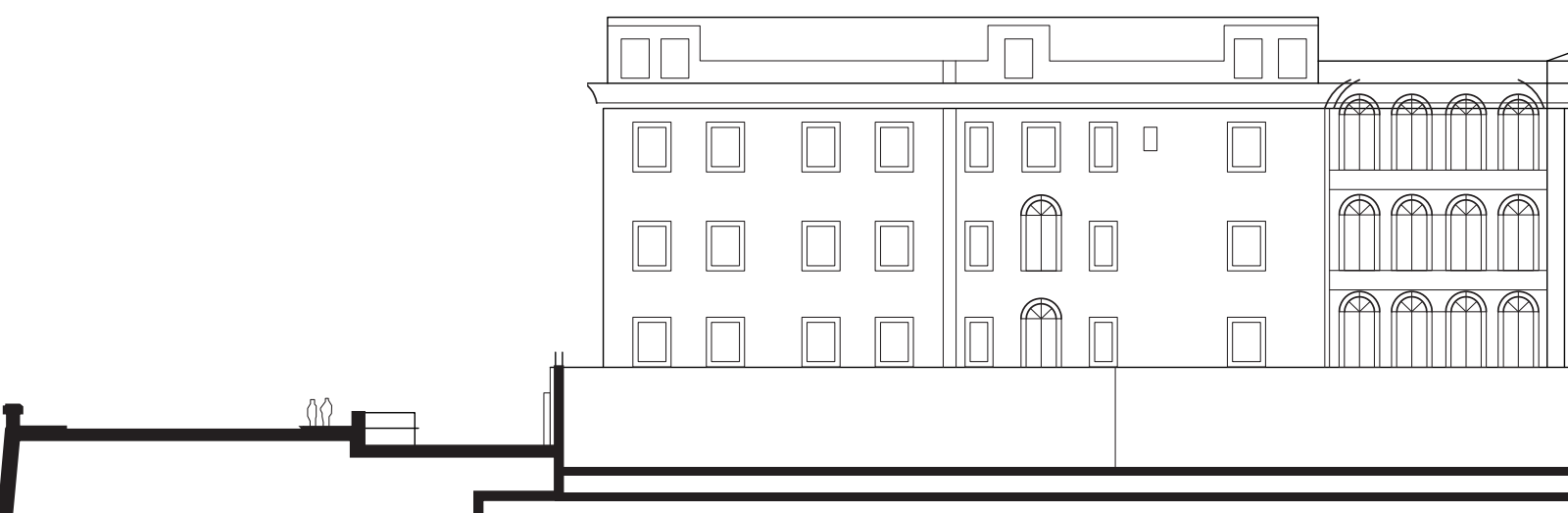

Figure A.22 Section A-A 


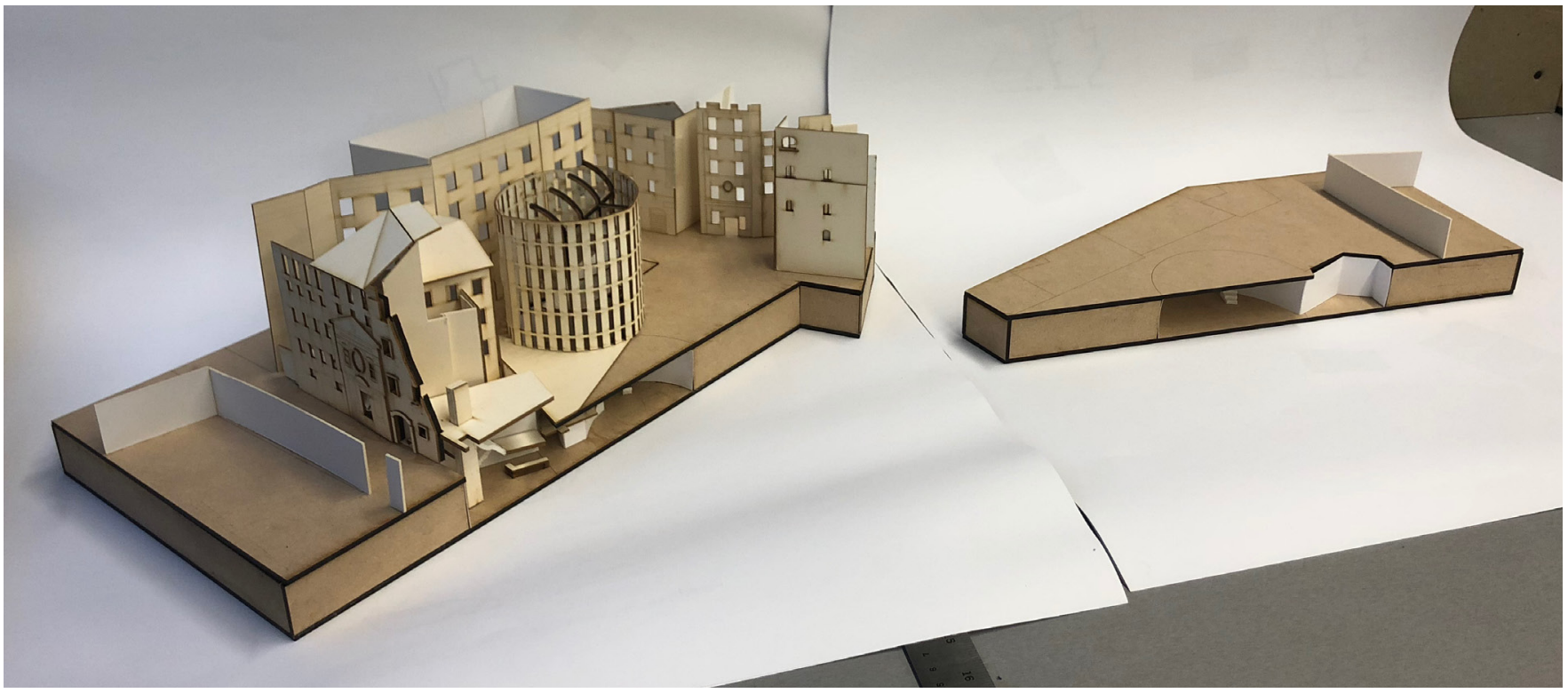

Figure A.23 Section A-A model

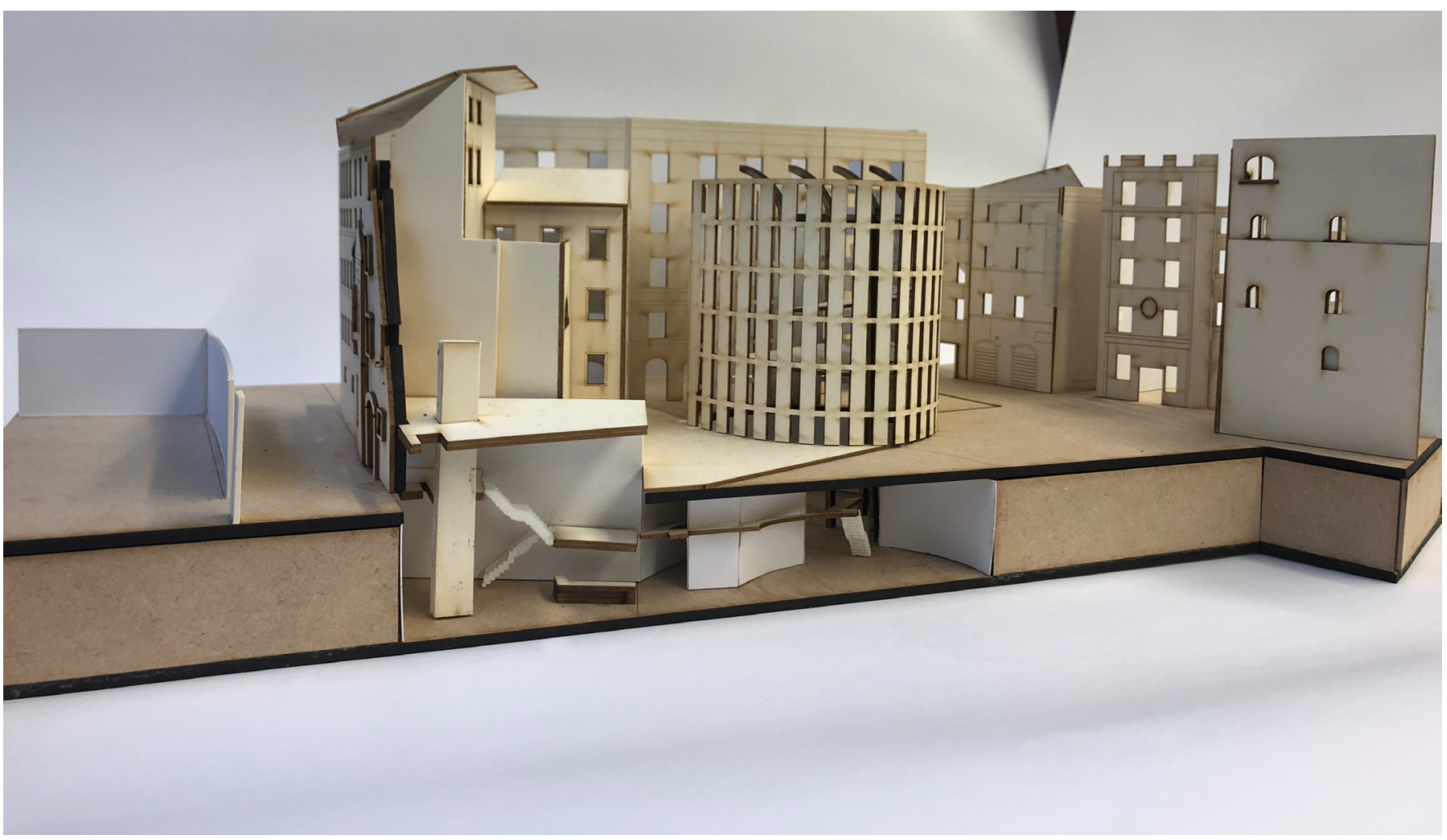

Figure A.24 Section A-A model from perspective 

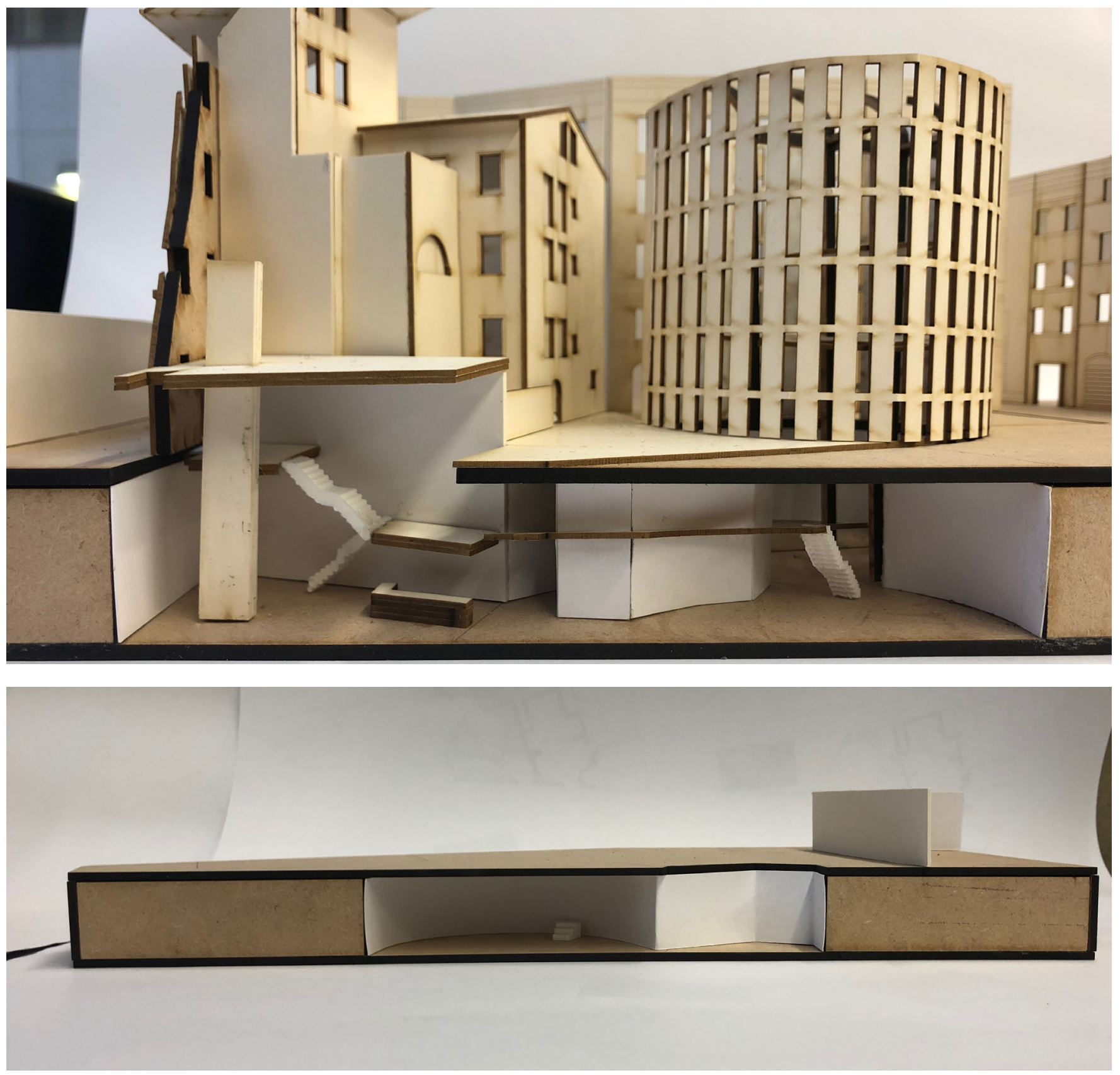

Figure A.25 Section A-A model, cafe and gallery volumes overlap without a need for a threshold

Figure A.26 The residual of Section A-A, cafe and gallery volumes appear discontinuous. There is an inherent need to imagine oneself at that scale when looking at a physical model, or on the other hand, we remain divorced from the architectural space and regard the model as an object. 


\section{B I B L I OGRAPHY}

Lytro Support, www.lytro.com/volumetracer\#video.

San Filippo Neri in Via Giulia, romafelix.com/sfilineri.htm.

Akten, Memo. "FIGHT! Virtual Reality Binocular Rivalry (VRBR). Memo Akten - Medium." Medium, Augmenting Humanity, 26 Mar. 2017, medium.com/@memoakten/fight-virtualreality-binocular-rivalry-89a0a91c2274.

Arkoun, Mohammed. Architecture Beyond Architecture: Spirituality and Architecture. archnet.org/system/publications/ contents/3690/original/DPT0018.pdf?1384775984.

Bachelard, Gaston, and M. Jolas. The Poetics of Space. Penguin Books, 2014.

Benjamin, Walter. The Work of Art in the Age of Mechanical Reproduction. Prism Key Press, 2010.

Bickhard, M. H., \& Richie, D. M. On the nature of representation: A case study of James Gibson's theory of perception. New York: Praeger. 1983.

Biocca, Frank, and Mark R. Levy. Communication in the Age of Virtual Reality. L. Erlbaum Associates, 1995.

Bleeckere, Sylvain de, and Sebastiaan Gerards. Narrative Architecture: a Designers Story. Routledge, 2017.

Boundless. "Boundless Psychology." Lumen, Open SUNY Textbooks, courses.lumenlearning.com/boundlesspsychology/chapter/attention/.

Brandolini, Sebastiano. Rome: New Architecture. Skira, 2008.

Cache, Bernard. Earth Moves: The Furnishing of Territories. Cambridge, MA: MIT Press. 1995, p140-2.
Carpo, Mario. The Second Digital Turn Design beyond Intelligence. The MIT Press, 2017.

"Chapel." Dictionary.com, www.dictionary.com/.

Crisman, Phoebe, and Mark L. Gillem. The Value of Design: Design Is at the Core of What We Teach and Practice. ACSA Press, 2009.

"Cubemap and Stereoscopic Images." Yulio Support Centre, yulio. zendesk.com/hc/en-us/articles/218813758-Cubemapand-Stereoscopic-Images.

Dai, Fan. Virtual Reality for Industrial Applications. Springer, 1998.

"Denise Scott Brown On the Past, Present and Future of VSBA's Groundbreaking Theories." ArchDaily, 7 Oct. 2016, www.archdaily.com/796821/denise-scott-brown-on-thepast-present-and-future-of-vsbas-groundbreakingtheories?ad_medium=widget\&ad_name=interviewsarticle-show.

Dunning, William V. Changing Images of Pictorial Space: a History of Spatial Illusion in Painting. Syracuse University Press, 1991.

Dyson, Frances. "Space", "Being" and other Fiction in the Domain of the Virtual." The Virtual Dimension. 1998, 27-45

Eco, Umberto. Travels in Hyper Reality: Essays. Harcourt Brace \& Company, 1990.

Edgerton, Samuel Y. The Mirror, the Window, and the Telescope: How Renaissance Linear Perspective Changed Our Vision of the Universe. Cornell University Press, 2009.

"Fixed Point Render Requirements." Yulio Support Centre, yulio. zendesk.com/hc/en-us/articles/219131167. 
Gombrich, E. H. Art and Illusion; a Study in the Psychology of Pictorial Representation. Pantheon Books, 1965.

Gordon, Ian E. Theories of Visual Perception. Taylor and Francis, 2004.

Hale, Jonathan A., and William W. Braham. Rethinking Technology: a Reader in Architectural Theory. Routledge, 2007.

"Heim: Essence of VR." The United States War on Drugs, web. stanford.edu/class/history34q/readings/Michael_Heim/ HeimEssenceVR.html.

James, William. The Principles of Psychology. Vol. 1-2, H. Holt, 1890.

Jonassen, David H. Handbook of Research for Educational Communications and Technology: a Project of the Association for Educational Communications and Technology. Macmillan Library Reference USA, 1996.

Juhani, Pallasmaa. The Eyes of the Skin: Architecture and the Senses. Wiley, 2014.

Kastrenakes, Jacob. "Google's Project Tango Is Shutting down Because ARCore Is Already Here." The Verge, The Verge, 15 Dec. 2017, www.theverge.com/2017/12/15/16782556/ project-tango-google-shutting-down-arcoreaugmented-reality.

Lam, William M.C., and Christopher Hugh. Ripman. Perception and Lighting as Formgivers for Architecture. Van Nostrand Reinhold, 1992.

Lang, Jon T. Designing for Human Behavior Architecture and the Behavioral Sciences. Dowden, Hutchinson \& Ross, 1980.

"Linear Perspective: Brunelleschi's Experiment." Khan Academy, Khan Academy, www.khanacademy.org/humanities/ renaissance-reformation/early-renaissance1/beginnersrenaissance-florence/v/linear-perspective-brunelleschis-experiement.
Logan, Robert K. Understanding New Media: Extending Marshall McLuhan. Peter Lang, 2016.

Merleau-Ponty, Maurice. Phenomenology of Perception. Forgotten Books, 2015.

Metzinger, Thomas. The Ego Tunnel. Basic Books, 2009.

Mine R.M., Brooks, F., Sequin, H.C., Moving Objects in Space: Exploiting Proprioception in In Virtual Environment Interaction, University of North Carolina at Chapel Hill, University of Berkeley, 2001.

Morris, Mark. "Digital space and analog place: A Shared Reality". Miami University

Morelli, Vincenzo. "Via Giulia in the cinquecento: From Giulio II to Sisto V", 2017. http://www.morelli.it/foglio/giulia/storia. html

Mullis, Alex. "How Does Virtual Reality Work?" Android Authority, 15 July 2016, www.androidauthority.com/virtual-realitywork-702049/.

Orr, Frank. Scale in Architecture. Chapman \& Hall, 1985.

Pérez-Gómez Alberto, and Louise Pelletier. Architectural Representation and the Perspective Hinge. MIT Press, 2000.

"Phenomenology." Stanford Encyclopedia of Philosophy, Stanford University, plato.stanford.edu/.

Picard, Max. The World of Silence. Eighth Day Press, 2002.

Pinchbeck, Daniel, and Ken Jordan. Exploring the Edge Realms of Consciousness: Liminal Zones, Psychic Science, and the Hidden Dimensions of the Mind. Evolver Editions, 2012. 
Politakis, Charalampos. Architectural Colossi and the Human Body: Buildings and Metaphors. Routledge, 2018.

Porter, Tom. The Architects Eye: Visualization and Depiction of Space in Architecture. E \& FN Spon, 1997.

"Program as Horizon - Frank Weiner, Meditations on Two Competition Projects, 2012." E2A Architects Zurich, www. e2a.ch/news/program-horizon\#/page1/.

Rae, Murray A. Architecture and Theology The Art of Place. Baylor University Press, 2017.

Rasmussen, Steen Eiler. Experiencing Architecture. MIT Press, 1959.

Sartre, Jean-Paul, and Philip Mairet. Sketch for a Theory of the Emotions. Routledge, 2014.

Schaik, Leon Van. Spatial Intelligence New Futures for Architecture. Wiley, 2008.

Sherman, William R. Understanding Virtual Reality: Interface, Application, and Design. MORGAN KAUFMANN, 2017. radekk76.

"Slavoj Zizek - The Reality of the Virtual -Part 1." YouTube, YouTube, 10 Dec. 2010, www.youtube.com/ watch?v=KdpudWL5i68. Some Characteristics. Routedge, 2007.

Stegers, Rudolf, and Dorothea Baumann. Sacred Buildings: a Design Manual. Birkhäuser, 2010.
Steinicke, Frank. Being Really Virtual Immersive Natives and the Future of Virtual Reality. Springer Berlin Heidelberg, 2016.

Temple, Nicholas. Disclosing Horizons: Architecture, Perspective and Redemptive Space. Routledge, 2007.

Tuan, Yi-Fu. Space and Place: the Perspective of Experience. University of Minnesota Press, 2014.

Vesely, Dalibor. Architecture in the Age of Divided Representation: the Question of Creativity in the Shadow of Production. The MIT Press, 2006.

Wang, Xiangyu, and Marc Aurel. Schnabel. Mixed Reality in Architecture, Design and Construction. Springer, 2010.

Weil, Simone, and Siân Miles. An Anthology. Penguin Books, 2005.

"What Is an FPR?" Yulio Support Centre, yulio.zendesk.com/hc/ en-us/articles/217173258-What-is-an-FPR-. Wittgenstein, Ludwig. Tractatus Logico-Philosophicus. Routledge, 1988. 